\title{
A detailed study of the rotating toroids in G31.41+0.31 and G24.78+0.08 ^
}

\author{
M. T. Beltrán ${ }^{1}$, R. Cesaroni ${ }^{1}$, R. Neri ${ }^{2}$, C. Codella ${ }^{3}$, R. S. Furuya ${ }^{4}$, L. Testi ${ }^{1}$, and L. Olmi ${ }^{3}$ \\ 1 INAF, Osservatorio Astrofisico di Arcetri, Largo E. Fermi 5, 50125 Firenze, Italy \\ e-mail: mbeltran@arcetri .astro.it \\ 2 IRAM, 300 rue de la Piscine, 38406 Saint Martin d'Hères, France \\ 3 Istituto di Radioastronomia, INAF, Sezione di Firenze, Largo E. Fermi 5, 50125 Firenze, Italy \\ ${ }^{4}$ Division of Physics, Mathematics, and Astronomy, California Institute of Technology, MS 105-24, Pasadena, \\ CA 91125 , USA
}

Received 17 November 2004 / Accepted 27 January 2005

\begin{abstract}
We present the results of high angular resolution millimeter observations of gas and dust toward G31.41+0.31 and G24.78+0.08, two high-mass star forming regions where four rotating massive toroids have been previously detected. The $\mathrm{CH}_{3} \mathrm{CN}(12-11)$ emission of the toroids in $\mathrm{G} 31.41+0.31$ and core $\mathrm{A} 1$ in $\mathrm{G} 24.78+0.08$ has been modeled assuming that it arises from a disk-like structure seen edge-on, with a radial velocity field. For G31.41+0.31 the model properly fits the data for a velocity $v_{\text {rot }} \simeq 1.7 \mathrm{~km} \mathrm{~s}^{-1}$ at the outer radius $R_{\text {out }} \simeq 13400 \mathrm{AU}$ and an inner radius $R_{\text {inn }} \simeq 1340 \mathrm{AU}$, while for core A1 in $\mathrm{G} 24.78+0.08$ the best fit is obtained for $v_{\text {rot }} \simeq 2.0 \mathrm{~km} \mathrm{~s}^{-1}$ at $R_{\text {out }} \simeq 7700 \mathrm{AU}$ and $R_{\text {inn }} \simeq 2300 \mathrm{AU}$. Unlike the rotating disks detected around less luminous stars, these toroids are not undergoing Keplerian rotation. From the modeling itself, however, it is not possible to distinguish between constant rotation or constant angular velocity, since both velocity fields suitably fit the data. The best fit models have been computed adopting a temperature gradient of the type $T \propto R^{-3 / 4}$, with a temperature at the outer radius $T_{\text {out }} \simeq 100 \mathrm{~K}$ for both cores. The $M_{\text {dyn }}$ needed for equilibrium derived from the models is much smaller than the mass of the cores, suggesting that such toroids are unstable and undergoing gravitational collapse. The collapse is also supported by the $\mathrm{CH}_{3}{ }^{13} \mathrm{CN}$ or $\mathrm{CH}_{3} \mathrm{CN}$ line width measured in the cores, which increases toward the center of the toroids. The estimates of $v_{\text {inf }}$ and $\dot{M}_{\text {acc }}$ are $\sim 2 \mathrm{~km} \mathrm{~s}^{-1}$ and $\sim 3 \times 10^{-2} M_{\odot} \mathrm{yr}^{-1}$ for G31.41+0.31, and $\sim 1.2 \mathrm{~km} \mathrm{~s}^{-1}$ and $\sim 9 \times 10^{-3} M_{\odot} \mathrm{yr}^{-1}$ for G24.78+0.08 A1. Such large accretion rates could weaken the effect of stellar winds and radiation pressure and allow further accretion on the star. The values of $T_{\text {rot }}$ and $N_{\mathrm{CH}_{3} \mathrm{CN}}$, derived by means of the RD method, for both G31.41+0.31 and the sum of cores A1 and A2 (core A of Codella et al. 1997, A\&A, 325, 282) in G24.78+0.08 are in the range 132-164 K and 2-8 $\times 10^{16} \mathrm{~cm}^{-2}$. For G31.41+0.31, the most plausible explanation for the apparent toroidal morphology seen in the lower $K$ transitions of $\mathrm{CH}_{3} \mathrm{CN}(12-11)$ is self-absorption, which is caused by the high optical depth and temperature gradient in the core.
\end{abstract}

Key words. ISM: individual: G31.41+0.31, G24.78+0.08 - ISM: molecules - radio lines: ISM - stars: formation

\section{Introduction}

The formation of massive stars represents a puzzle from a theoretical point of view. Unlike low-mass stars, they are believed to reach the zero-age main sequence still deeply embedded in their parental cores: in particular, Palla \& Stahler (1993) predict that this occurs for stellar masses in excess of $8 M_{\odot}$. Once the star has ignited hydrogen burning, further accretion should be inhibited by radiation pressure and powerful stellar winds, with the consequence that stars more massive than $8 M_{\odot}$ should not exist. Two formation scenarios have been proposed to solve this paradox: non-spherical accretion (Nakano et al. 1995; Jijina \& Adams 1996) and merging of lower mass stars (Bonnell et al. 1998). Discriminating between these two models represents a challenging observational goal.

* Based on observations carried out with the IRAM Plateau de Bure Interferometer. IRAM is supported by INSU/CNRS (France), MPG (Germany) and IGN (Spain).
In this context, the detection of disks would strongly favour the accretion scenario, as naively one would expect that random encounters between merging stars do not lead to axially symmetric structures since the disks associated with the lower mass stars should be destroyed during the merging process. On the contrary, conservation of angular momentum is bound to cause flattening and rotation of the infalling material, thus producing disk-like bodies. Indeed, circumstellar disks have been detected in low-mass stars and found to undergo Keplerian rotation (Simon et al. 2000). Similar evidence has been found in a few high-mass Young Stellar Objects (YSOs), but in most cases the angular resolution was insufficient to assess the presence of a disk unambiguously. Only a few bona fide examples are known (see Cesaroni 2002) and all of these are associated with moderately massive stars (B1-B0). This is not sufficient to understand the role of disks in the formation of even more 
massive stars and establish the relevance of accretion for this process.

With this in mind, we have decided to perform a search for disks in high-mass YSOs by means of high angular resolution observations. We have selected luminous $\left(L_{\mathrm{bol}}>10^{4} L_{\odot}\right)$ objects with typical signposts of massive star formation such as water masers and ultracompact (UC) HII regions. The first objects selected for this project are G31.41+0.31 and $\mathrm{G} 24.78+0.08$. The former is a well-studied hot core located at $7.9 \mathrm{kpc}$ (Cesaroni et al. 1994, 1998), where preliminary evidence of a rotating massive disk oriented perpendicularly to a bipolar outflow has been reported in Cesaroni et al. (1994) and Olmi et al. (1996a). The latter is a cluster of massive (proto)stars with a distance of $7.7 \mathrm{kpc}$, where recently Furuya et al. (2002) have detected a pair of cores, each of these associated with a compact bipolar outflow. On the basis of previous experience with this type of object (see e.g. Cesaroni et al. 1999), $\mathrm{CH}_{3} \mathrm{CN}$ has been used as disk tracer. This is a lowabundance molecule which is excited in very dense regions. Therefore, searching for disks requires not only high angular resolution, but also great sensitivity given the faintness of the lines observed. The first result derived from the analysis of the high angular resolution data has been the detection of clear velocity gradients perpendicular to the axis of the molecular outflows in the cores (see Fig. 2 in Beltrán et al. 2004, hereafter Paper I), which points out the presence of rotating structures in the hot cores: one in G31.41+0.31 and three in G24.78+0.08. In Paper I, we referred to these rotating structures as "disks"; however, their sizes ( 8000-16000 AU in diameter) and masses $\left(\sim 80-500 M_{\odot}\right)$ make them remarkably different from the geometrically thin circumstellar disks seen in low-mass YSOs, where the mass of the disk is much less than that of the star. Thus, these structures are more toroidal, and it is plausible to interpret such toroids as "circumcluster" disks, in the sense that they may host not just a single star, but a whole cluster. The rotating toroidal structures detected in Paper I are unstable as suggested by the fact that the mass of the cores is much larger than the dynamical mass needed for equilibrium. The main finding of the preliminary results of such a study is that nonspherical accretion is a viable mechanism to form high-mass stars.

In this article we present a full report on the results obtained in Paper I, and a complete analysis of the data. We thoroughly analyze all the molecular lines detected toward the cores, the gas and dust distribution, and the physical parameters. We study in detail the kinematics of the cores and model the gas emission by assuming that it arises from a disk-like structure with an internal radial velocity field, and by adopting power-law distributions for the velocity and temperature of the emitting gas.

\section{Observations}

\subsection{BIMA observations}

Millimeter observations of G24.78+0.08 were carried out with the BIMA array ${ }^{1}$ in the A, B, C, and D configurations between

\footnotetext{
1 The BIMA array is operated by the Berkeley-Illinois-Maryland Association with support from the National Science Foundation.
}

2002 September and 2003 February (see Table 1). The digital correlator was configured to observe simultaneously the continuum emission and some molecular lines. Details of the observations are given in Table 1 . The phase center was located at $\alpha(\mathrm{J} 2000)=18^{\mathrm{h}} 36^{\mathrm{m}} 12^{\mathrm{s}} .659, \delta(\mathrm{J} 2000)=-07^{\circ} 12^{\prime} 10^{\prime} \cdot 15$. The bandpass calibration was carried out on $3 \mathrm{C} 345$. Amplitude and phase were calibrated by observations of 1743-048, whose flux density of $4.4 \mathrm{Jy}$ at $3.20 \mathrm{~mm}$ (A and B configurations) and of $4.7 \mathrm{Jy}$ at $3.16 \mathrm{~mm}$ (C and $\mathrm{D}$ configurations) was determined relative to Uranus, or Mars for the B configuration observations. The data were calibrated and analyzed using standard procedures in the MIRIAD software package. We subtracted the continuum from the line emission directly in the $(u, v)$ domain in order to reduce non linearity effects in the deconvolution, and thus any amplification of errors induced in this process.

\subsection{PdBI observations}

Interferometric observations of G31.41+0.31 and G24.78+0.08 were carried out with the IRAM Plateau de Bure Interferometer (PdBI) in the most extended configuration on 2003 March 16. By using the dual frequency capabilities of the PdBI we observed the continuum and molecular lines simultaneously at 3.3 and $1.4 \mathrm{~mm}$. The receivers were tuned single-sideband at $3.3 \mathrm{~mm}$ and double-sideband at $1.4 \mathrm{~mm}$. The correlator was centered at $92.0475 \mathrm{GHz}$ in the lower sideband at $3.3 \mathrm{~mm}$ and at $220.5250 \mathrm{GHz}$ in the upper sideband at $1.4 \mathrm{~mm}$. The frequency setup of the correlator and the list of the observed molecular lines are shown in Table 2. The eight units of the correlator were placed in such a way that a frequency range free of lines could be used to measure the continuum flux. The phase center was set to the position $\alpha(\mathrm{J} 2000)=18^{\mathrm{h}} 47^{\mathrm{m}} 34.330$, $\delta(\mathrm{J} 2000)=-01^{\circ} 12^{\prime} 46^{\prime \prime} 50$ for G31.41+0.31, and to $\alpha(\mathrm{J} 2000)=$ $18^{\mathrm{h}} 36^{\mathrm{m}} 12^{\mathrm{s}} .661, \delta(\mathrm{J} 2000)=-07^{\circ} 12^{\prime} 10^{\prime} \cdot 15$ for $\mathrm{G} 24.78+0.08$. The bandpass of the receivers was calibrated by observations of the quasar 3C 273. Amplitude and phase calibrations were achieved by monitoring 1741-038, whose flux densities of $4.63 \mathrm{Jy}$ and $2.99 \mathrm{Jy}$ at $3.3 \mathrm{~mm}$ and $1.4 \mathrm{~mm}$, respectively, were determined relative to MWC349. The data were calibrated and analyzed with the GILDAS software package developed at IRAM and Observatoire de Grenoble. We subtracted the continuum from the line emission directly in the $(u, v)$-domain. The synthesized CLEANed beams for maps made using natural weighting were $2^{\prime \prime} .7 \times 1^{\prime \prime} .2$ at PA $=-174^{\circ}(3.3 \mathrm{~mm})$ and $1^{\prime \prime} .1 \times 0.0^{\prime} 5$ at $\mathrm{PA}=-170^{\circ}(1.4 \mathrm{~mm})$ for $\mathrm{G} 31.41+0.31$, and 3.0.' $1 \times 1^{\prime \prime} .2$ at PA $=-177^{\circ}(3.3 \mathrm{~mm})$ and $1^{\prime \prime} .2 \times 0.0^{\prime} 5$ at $\mathrm{PA}=-174^{\circ}(1.4 \mathrm{~mm})$ for $\mathrm{G} 24.78+0.08$. Note that the G24.78+0.04 BIMA and PdBI continuum data at $3 \mathrm{~mm}$ were merged in the $(u, v)$-domain (see next section) and the synthesized CLEANed beam for the resulting map was $1 . \prime 3 \times 0.9$ at $\mathrm{PA}=0^{\circ}$.

One of the lines observed and clearly detected toward both regions is ${ }^{13} \mathrm{CO}(2-1)$. However, in both cases the line profile exhibits a strong lack of emission at the center, probably due to a missing flux problem since the interferometer might have filtered out part of the extended emission as suggested by 
Table 1. Parameters of the BIMA observations.

\begin{tabular}{lccccccc}
\hline \hline \multirow{2}{*}{ Observation } & Configuration & $\begin{array}{c}\text { Frequency } \\
(\mathrm{GHz})\end{array}$ & $\begin{array}{c}\text { Synthesized beam } \\
\text { (arcsec) }\end{array}$ & $\begin{array}{c}\text { PA } \\
(\mathrm{deg})\end{array}$ & $\begin{array}{c}\text { Bandwidth } \\
(\mathrm{MHz})\end{array}$ & $\begin{array}{c}\text { Spectral resolution } \\
\left(\mathrm{km} \mathrm{s}^{-1}\right)\end{array}$ & $\begin{array}{c}\text { rms noise }^{a} \\
\left(\mathrm{mJy} \mathrm{beam}^{-1}\right)\end{array}$ \\
\hline $3.20 \mathrm{~mm}$ continuum & $\mathrm{A}, \mathrm{B}$ & 93.55 & $1.28 \times 0.94$ & 0 & 1200 & - & 1 \\
$\mathrm{CH}_{3} \mathrm{CN}(5-4)$ & $\mathrm{A}, \mathrm{B}$ & 91.98 & $0.83 \times 0.60$ & 61 & 25 & 0.31 & 50 \\
$3.16 \mathrm{~mm}$ continuum & $\mathrm{C}, \mathrm{D}$ & 94.75 & $19.8 \times 16.3$ & -2 & 800 & - & 3 \\
$\mathrm{~N}_{2} \mathrm{H}^{+}(1-0)$ & $\mathrm{C}, \mathrm{D}$ & 93.18 & $14.3 \times 13.0$ & 16 & 25 & 0.31 & 100 \\
$\mathrm{C}^{34} \mathrm{~S}(2-1)$ & $\mathrm{C}, \mathrm{D}$ & 96.41 & $12.6 \times 13.5$ & 14 & 25 & 0.31 & 100 \\
$\mathrm{CH}_{3} \mathrm{OH}\left(2_{k}-1_{k}\right)$ & $\mathrm{C}, \mathrm{D}$ & 96.74 & $14.0 \times 12.6$ & 12 & 25 & 0.31 & 100 \\
\hline
\end{tabular}

${ }^{a}$ For the molecular line observations the $1-\sigma$ noise is per channel.

Table 2. Frequency setups used for the molecular lines observed with the IRAM PdBI.

\begin{tabular}{lccccc}
\hline \multicolumn{1}{c}{ Line } & $\begin{array}{c}\text { Center frequency } \\
(\mathrm{MHz})\end{array}$ & $\begin{array}{c}\text { Bandwidth } \\
(\mathrm{MHz})\end{array}$ & $\begin{array}{c}\text { Spectral resolution } \\
(\mathrm{MHz})\end{array}$ & $\begin{array}{c}\text { rms noise }^{a} \\
\left(\mathrm{~km} \mathrm{~s}^{-1}\right)\end{array}$ & \begin{tabular}{c}
$\left(\mathrm{mJy} \mathrm{beam}^{-1}\right)$ \\
\hline $\mathrm{CH}_{3} \mathrm{CN}(5-4), \mathrm{CH}_{3}{ }^{13} \mathrm{CN}(5-4)$
\end{tabular} \\
$\mathrm{CH}_{3}{ }^{13} \mathrm{CN}(5-4)$ & 91987.094 & 80 & 0.156 & 0.5 & 12 \\
$\mathrm{CH}_{3} \mathrm{CN}(5-4) v_{8}=1$ & 91913.398 & 320 & 2.5 & 8.1 & 3 \\
$\mathrm{CH}_{3} \mathrm{CN}(5-4) v_{8}=1$ & 92353.438 & 160 & 0.625 & 2.0 & 7 \\
$\mathrm{CH}_{3} \mathrm{CN}(12-11)$ & 92175.484 & 320 & 2.5 & 8.1 & 3 \\
$\mathrm{CH}_{3} \mathrm{CN}(12-11), \mathrm{CH}_{3}{ }^{13} \mathrm{CN}(12-11)$ & 220747.266 & 80 & 0.156 & 0.2 & 50 \\
$\mathrm{CH}_{3} \mathrm{CN}(12-11), \mathrm{CH}_{3}{ }^{13} \mathrm{CN}(12-11)$ & 220679.297 & 80 & 0.156 & 0.2 & 50 \\
$\mathrm{CH}_{3} \mathrm{CN}(12-11), \mathrm{CH}_{3}{ }^{13} \mathrm{CN}(12-11),{ }^{13} \mathrm{CO}(2-1)$ & 220594.422 & 160 & 1.25 & 1.7 & 20 \\
\hline
\end{tabular}

${ }^{a}$ For the molecular line observations the $1-\sigma$ noise is per channel.

the single-dish spectra $(\mathrm{G} 31.41+0.31$ : Cesaroni, private communication; G24.78+0.08: Cesaroni et al. 2003). In addition, although the line exhibits prominent wings, it has been impossible to disentangle the core emission (at a systemic velocity) from the high-velocity emission to derive the parameters of the outflowing material. Therefore, the emission of ${ }^{13} \mathrm{CO}(2-1)$ has not been analyzed in the paper.

\subsection{Merging the BIMA and $\mathrm{PdBI}$ data for $\mathrm{G} 24.78+0.08$}

The BIMA (A and B configuration) and PdBI continuum data of $\mathrm{G} 24.78+0.08$ at $3 \mathrm{~mm}$ were merged in the $(u, v)$-domain. The projected baseline lengths range from $\sim 3.8$ to $416 \mathrm{k} \lambda$ for the BIMA data, and from $\sim 4.8$ to $127 \mathrm{k} \lambda$ for the PdBI data. The BIMA $(u, v)$-data were first converted to FITS format using a procedure available in the MIRIAD software package, and then converted to GILDAS format using a procedure available in the GILDAS software package. All the BIMA data were then merged together to create a single $(u, v)$-table using the task $u v \_$merge of GILDAS, and the relative weights of the data were divided by $1 \times 10^{6}$ so that the weight density of the BIMA data matches that of the PdBI data. Finally, the PdBI $(u, v)$-data were merged with the already created $\operatorname{BIMA}(u, v)$-table.

\section{G31.41+0.31}

\subsection{Results}

\subsubsection{Continuum emission}

In Fig. 1 we show the PdBI maps of the 3.3 and $1.4 \mathrm{~mm}$ continuum emission toward the hot core G31.41+0.31.
The continuum dust emission is marginally resolved at both wavelengths and shows a compact source plus an extended envelope. With the BIMA interferometer, Wyrowski et al. (2001) have mapped a similar structure at $1.4 \mathrm{~mm}$. The position and fluxes at 3.3 and $1.4 \mathrm{~mm}$, and the deconvolved size of the source, measured as the geometric mean of the major and minor axes of the $50 \%$ of the peak contour at $1.4 \mathrm{~mm}$, are given in Table 3. The flux densities of $0.67 \mathrm{Jy}$ at $3.3 \mathrm{~mm}$ and $4.4 \mathrm{Jy}$ at $1.4 \mathrm{~mm}$ are consistent with the values of $0.28 \mathrm{Jy}$ at $3.4 \mathrm{~mm}$ and of $4.0 \mathrm{Jy}$ at $1.3 \mathrm{~mm}$ measured by Maxia et al. (2001) with the Owens Valley Radio Observatory (OVRO) interferometer. Hatchell et al. (2000) have measured a flux of $4.9 \mathrm{Jy}$ at $1.35 \mathrm{~mm}$ inside the 22" James Clerk Maxwell Telescope (JCMT) beam. The fact that the continuum flux seen with a single dish is not much higher than that measured with the interferometers seems to indicate that the source is very centrally peaked, and that there is no significant fraction of the total flux filtered out by the interferometers. In Paper I we derived a core mass of $490 M_{\odot}$ from the millimeter continuum emission assuming a mass opacity of $\simeq 0.02 \mathrm{~cm}^{-2} \mathrm{~g}^{-1}$ at $1.4 \mathrm{~mm}$ for a gas-to-dust ratio of 100 and a temperature of $230 \mathrm{~K}$ (Olmi et al. 1996a).

\subsection{2. $\mathrm{CH}_{3} \mathrm{CN}$ and $\mathrm{CH}_{3}{ }^{13} \mathrm{CN}$}

Figure 2 shows the spectra of the $\mathrm{CH}_{3} \mathrm{CN}$ and $\mathrm{CH}_{3}{ }^{13} \mathrm{CN}$ (12-11) and (5-4) lines toward G31.41+0.31. The first vibrational state above the ground of the $\mathrm{CH}_{3} \mathrm{CN}$ (5-4) line, which is denoted $v_{8}=1$, is also shown in this figure. The spectra have been taken integrating the emission over the $3 \sigma$ contour level area. Figure 3 shows the maps of the $\mathrm{CH}_{3} \mathrm{CN}$ (12-11) emission averaged under the $K=1$ and 2 components and under the 
Table 3. Positions, flux densities and sizes at millimeter wavelengths for the cores in G31.41+0.31 and G24.78+0.08.

\begin{tabular}{|c|c|c|c|c|c|c|c|c|}
\hline \multirow[b]{2}{*}{ Core } & \multicolumn{2}{|c|}{ " Position $^{a}$} & \multirow[b]{2}{*}{$\begin{array}{c}I_{v}^{\text {peak }}(3.3 \mathrm{~mm}) \\
\left(\mathrm{Jy} \mathrm{beam}^{-1}\right)\end{array}$} & \multirow[b]{2}{*}{$\begin{array}{c}S_{v}(3.3 \mathrm{~mm}) \\
(\mathrm{Jy})\end{array}$} & \multirow[b]{2}{*}{$\begin{array}{c}I_{v}^{\text {peak }}(1.4 \mathrm{~mm}) \\
\left(\mathrm{Jy} \mathrm{beam}^{-1}\right)\end{array}$} & \multirow[b]{2}{*}{$\begin{array}{c}S_{v}(1.4 \mathrm{~mm}) \\
(\mathrm{Jy})\end{array}$} & \multicolumn{2}{|c|}{ Source diameter $^{b}$} \\
\hline & $\begin{array}{c}\alpha(\mathrm{J} 2000) \\
\mathrm{h} \mathrm{m} \mathrm{s}\end{array}$ & $\begin{array}{c}\delta(\mathrm{J} 2000) \\
\circ, \prime \prime\end{array}$ & & & & & $\begin{array}{c}\theta_{s} \\
(\operatorname{arcsec})\end{array}$ & $\begin{array}{c}\theta_{s} \\
(\mathrm{AU})\end{array}$ \\
\hline G31 & 184734.32 & -011245.9 & 0.252 & 0.674 & 1.3 & 4.4 & 1.1 & 8700 \\
\hline G24 A1 & 183612.57 & -071210.9 & $0.086^{c}$ & $0.211^{d}$ & 0.191 & 0.882 & 1.2 & 9200 \\
\hline G24 A2 & 183612.49 & -071210.0 & $0.086^{c}$ & $0.211^{d}$ & 0.125 & 0.574 & 0.9 & 7000 \\
\hline G24 B & $183612.66^{e}$ & $-071215.2^{e}$ & 0.016 & 0.040 & $<0.020^{f}$ & $<0.020^{f}$ & $1.5^{g}$ & $11500^{g}$ \\
\hline $\mathrm{G} 24 \mathrm{C}$ & 183613.12 & -071207.4 & 0.005 & 0.005 & 0.056 & 0.100 & 0.9 & 7000 \\
\hline
\end{tabular}

${ }^{a}$ Position of the $1.4 \mathrm{~mm}$ emission peak.

${ }^{b}$ Deconvolved geometric mean of the major and minor axes of the $50 \%$ of the peak contour at $1.4 \mathrm{~mm}$.

${ }^{c}$ Peak intensity of core A (cores A1 and A2 together; see Sect. 4.1.2).

${ }^{d}$ Flux density of core A (cores A1 and A2 together; see Sect. 4.1.2).

${ }^{e}$ Source not detected at $1.4 \mathrm{~mm}$. Position corresponds to the $3.3 \mathrm{~mm}$ emission peak.

${ }^{f}$ Upper limit for nondetected source is $3 \sigma$.

${ }^{g}$ Deconvolved geometric mean of the major and minor axes of the $50 \%$ of the peak contour at $3.3 \mathrm{~mm}$.

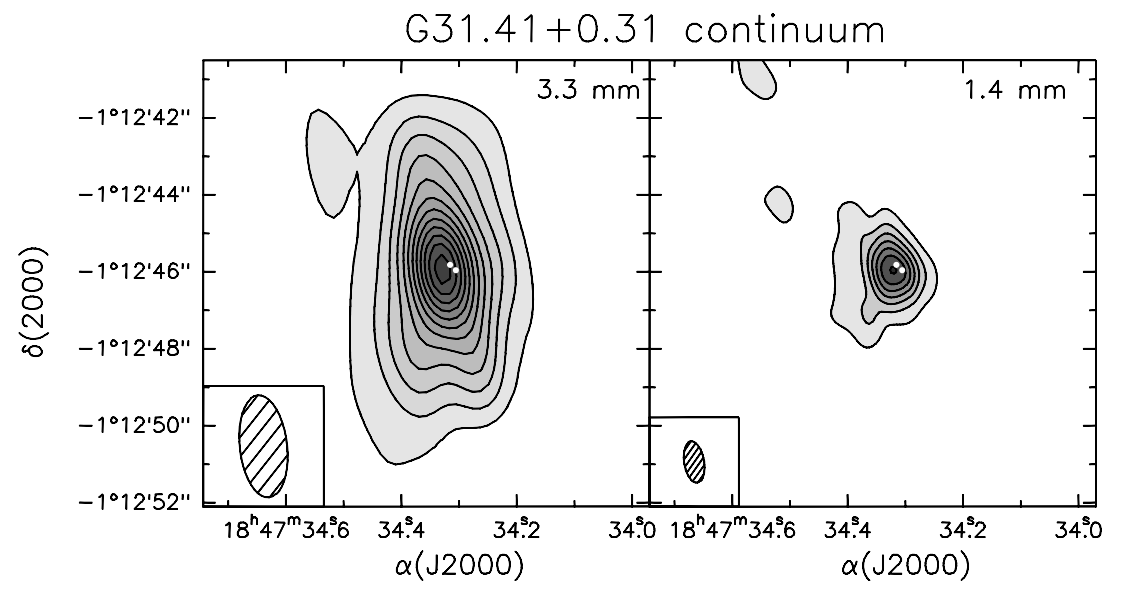

Fig. 1. PdBI map of the $3.3 \mathrm{~mm}$ (left panel) and the $1.4 \mathrm{~mm}$ (right panel) continuum emission toward the core of G31.41+0.31. The contour levels range from 20 to $240 \mathrm{mJy}_{\text {beam }}^{-1}$ in steps of $20 \mathrm{mJy}_{\text {beam }}^{-1}$ for the $3.3 \mathrm{~mm}$ map, and from 100 to $1300 \mathrm{mJy}^{\mathrm{beam}}{ }^{-1}$ in steps of $200 \mathrm{mJy} \mathrm{beam}^{-1}$ for the $1.4 \mathrm{~mm}$ map. The synthesized beam is shown in the lower left-hand corner. The white dots mark the position of the $7 \mathrm{~mm}$ continuum emission peaks detected by Hofner (private communication).

$K=8$ component, and the $\mathrm{CH}_{3}{ }^{13} \mathrm{CN}$ (12-11) emission averaged under the $K=1$ and 2 components. Figure 4 shows the maps of the $\mathrm{CH}_{3} \mathrm{CN}$ (5-4) and the $\mathrm{CH}_{3}{ }^{13} \mathrm{CN}$ (5-4) emission averaged under the $K=0$ to 4 components, and the $\mathrm{CH}_{3} \mathrm{CN}$ (5-4) $v_{8}=1$ emission averaged under all observed transitions.

Several $K$-components of the different rotational transitions of methyl cyanide are clearly detected toward G31.41+0.31 as can be seen in Fig. 2. We fitted multiple $K$-components of a given rotational transition by fixing their separation in frequency to the laboratory values (see Lovas 1992 and references therein) and forcing their line width to be identical. As different $K$-components of a same rotational transition fall in different PdBI correlator units with different spectral resolutions, sometimes we did not fit simultaneously all of the lines for a given rotational transition, but groups of them. The groups were of 5 lines at maximum because CLASS, which is the software package used to analyze the lines, only allows to fit 5 lines simultaneously. In addition, in a few cases, the line width in the fit was fixed to the value obtained for other $K$-components of the same transition; otherwise, due to the low spectral resolution with which those transitions have been observed, the line widths obtained when fitting the profiles were unrealistically wide. The fact of fixing the line width for groups of lines with different $K$-components fitted simultaneously does not affect significantly the results and conclusions derived from the analysis of the lines. The profiles show no evidence of selfabsorption, are quite symmetric and can be reasonably fitted by a Gaussian profile. The line parameters of the ground state transitions of $\mathrm{CH}_{3} \mathrm{CN}$ and $\mathrm{CH}_{3}{ }^{13} \mathrm{CN}$ are given in Table 4, while those of the $v_{8}=1$ are given in Table 5. Note that in some cases the lines are so blended with other methyl cyanide components (e.g. the $\mathrm{CH}_{3}{ }^{13} \mathrm{CN} K=0$ with $\mathrm{CH}_{3} \mathrm{CN} K=5$ for the (12-11) transition), or with other molecular lines such as ${ }^{13} \mathrm{CO}(2-1)$, that it has been impossible to derive their parameters. As can be seen in Tables 4 and 5 there is a good agreement between the systemic velocities derived from all transitions $\left(V_{\mathrm{LSR}} \simeq 97 \mathrm{~km} \mathrm{~s}^{-1}\right)$. Regarding the line widths, we found a similar value, $F W H M \simeq 8 \mathrm{~km} \mathrm{~s}^{-1}$, for $\mathrm{CH}_{3} \mathrm{CN}(12-11), \mathrm{CH}_{3}{ }^{13} \mathrm{CN}$ (12-11), and $\mathrm{CH}_{3} \mathrm{CN}$ (5-4) $v_{8}=1$. On the other hand, for 
$\mathrm{CH}_{3} \mathrm{CN}$ (5-4) and $\mathrm{CH}_{3}{ }^{13} \mathrm{CN}$ (5-4) we found values of $\sim 10$ and $\sim 6 \mathrm{~km} \mathrm{~s}^{-1}$, respectively. Assuming that the line profiles are Gaussian, the ratio of the observed line width (broadened by the line optical depth) and the intrinsic one, can be written as

$$
\frac{\Delta V_{\mathrm{obs}}}{\Delta V_{\mathrm{int}}}=\frac{1}{\sqrt{\ln 2}} \sqrt{-\ln \left[-\frac{1}{\tau_{0}} \ln \left(\frac{1+\mathrm{e}^{-\tau_{0}}}{2}\right)\right]},
$$

where $\tau_{0}$ is the optical depth at the center of the line. In practice, Eq. (1) gives the line width ratio between an optically thick and an optically thin transition of the same species, where $\tau_{0}$ corresponds to the optical depth of the thick line. One may hence apply Eq. (1) to the $K$ lines of $\mathrm{CH}_{3} \mathrm{CN}$ (5-4) and $\mathrm{CH}_{3}{ }^{13} \mathrm{CN}$ (5-4). The optical depth $\tau_{0}$ can be derived from the brightness temperature ratio between lines of the two species with the same $K$ (assuming a relative abundance $\left[\mathrm{CH}_{3} \mathrm{CN}\right] /\left[\mathrm{CH}_{3}{ }^{13} \mathrm{CN}\right]=50$; see Wilson \& Rood 1994): using this in Eq. (1) one obtains the line width ratio between $\mathrm{CH}_{3} \mathrm{CN}$ and $\mathrm{CH}_{3}{ }^{13} \mathrm{CN}$ expected if line broadening is due only to optical depth effects. Such a ratio turns out to range between 1.8 and 2.1 , roughly consistent with that measured directly from the line profiles: $1.6 \pm 0.02$. We conclude that the $\mathrm{CH}_{3} \mathrm{CN}$ lines are wider than the $\mathrm{CH}_{3}{ }^{13} \mathrm{CN}$ ones as a consequence of the larger optical depth, although one cannot exclude that different methyl cyanide transitions are tracing gas with slightly different physical conditions as well.

As can be seen in Fig. 1 of Paper I, and in Fig. 3, the $\mathrm{CH}_{3} \mathrm{CN} K=1$ and 2 components seem to trace a toroidal structure with the millimeter continuum emission peaking at the central dip. To explain this morphology two possible scenarios are suggested: (i) a dramatic $\mathrm{CH}_{3} \mathrm{CN}$ abundance drop in the central region of the core; or (ii) self-absorption, due to the high optical depth at the center of the core and the existence of a temperature gradient. Figures 3 and 5 show that the different $K$-components do not trace the same material, and that for large values of $K$ the $\mathrm{CH}_{3} \mathrm{CN}$ emission peaks closer to the center. In fact, as shown in Fig. 5, the $K=8$ emission fills the central dip visible in the $K=0,1$ and 2 emission. The $\mathrm{CH}_{3}{ }^{13} \mathrm{CN}$ $K=1$ and 2 emission is also found closer to the center than the $\mathrm{CH}_{3} \mathrm{CN} \mathrm{K}=1$ and 2 emission. Thus, the lack of emission at the center of the core occurs only for the lower $K$-components of $\mathrm{CH}_{3} \mathrm{CN}$, unlike the higher $K$-components, $\mathrm{CH}_{3}{ }^{13} \mathrm{CN}$ and continuum emission. This indicates that the $\mathrm{CH}_{3} \mathrm{CN}$ molecules are not destroyed at the center of the core. As can be seen in Fig. 2, the $\mathrm{CH}_{3} \mathrm{CN}(12-11) \mathrm{K}=0$ to 4 lines show similar intensities, suggestive of high optical depth. Note that low- $K$ transitions of $\mathrm{CH}_{3}{ }^{13} \mathrm{CN}$, which have excitation energies very similar to those of the corresponding $\mathrm{CH}_{3} \mathrm{CN} \mathrm{K}$ lines, trace a slightly inner region: this is an opacity effect as the less abundant isotopomer traces higher column densities. In order to confirm a possible opacity effect, we computed the optical depth at each point using the $\mathrm{K}=2$ components of $\mathrm{CH}_{3} \mathrm{CN}$ and $\mathrm{CH}_{3}{ }^{13} \mathrm{CN}$, assuming that $\mathrm{CH}_{3} \mathrm{CN}$ is optically thick and that the excitation temperature is the same for both transitions. A map of the optical depth of the $\mathrm{CH}_{3} \mathrm{CN}$ main species, $\tau_{12}$, shows that the opacity increases toward the center of the core: it ranges from 10 to 50 for $K=2$, and from 5 to 20 for $K=6$. In conclusion, the most
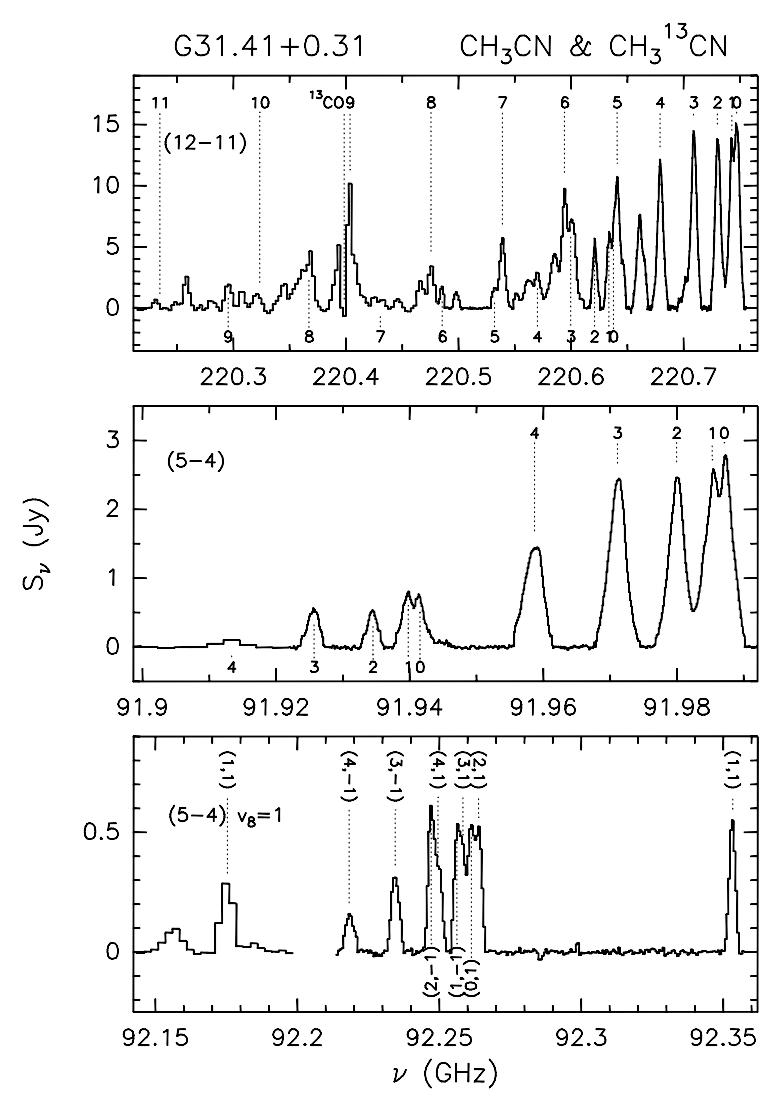

Fig. 2. Methyl cyanide spectra integrated over the $3 \sigma$ contour level area toward G31.41+0.31 as seen with the PdBI. We show in the top $\mathrm{CH}_{3} \mathrm{CN}$ (12-11), in the middle $\mathrm{CH}_{3} \mathrm{CN}$ (5-4), and in the bottom $\mathrm{CH}_{3} \mathrm{CN}(5-4)$ vibrationally excited $\left(v_{8}=1\right)$. Different $K$ numbers (top and middle panels) are marked with dashed lines in the upper (lower) part of each spectra in the case of $\mathrm{CH}_{3} \mathrm{CN}\left(\mathrm{CH}_{3}{ }^{13} \mathrm{CN}\right)$. Note that different $K$-components of a same transition have different spectral resolution because of the different spectral resolution of the correlator units (see Table 3).

plausible explanation for the apparent toroidal geometry of the $\mathrm{CH}_{3} \mathrm{CN}$ map in Fig. 3 is self-absorption, which is caused by the high optical depth and the temperature gradient in the core. In Sect. 3.2.1 we discuss in more detail this temperature gradient.

\subsection{Discussion}

\subsubsection{Temperature gradient}

In Sect. 3.1.2 we have illustrated the existence of a temperature gradient in the G31.41+0.31 core. Here we want to estimate the temperature and its variation across the core. Following Olmi et al. (1993) we derived estimates for the rotational temperature, $T_{\text {rot }}$, and the total methyl cyanide column density, $N_{\mathrm{CH}_{3} \mathrm{CN}}$, by means of the rotation diagram (RD) method, which assumes that the molecular levels are populated according to LTE conditions at a single temperature $T_{\text {rot }}$. In the high density limit where level populations are thermalised, one expects that $T_{\text {rot }}=T_{\mathrm{k}}$, the kinetic temperature. The $\mathrm{CH}_{3} \mathrm{CN}$ ground level transitions appear to be optically thick, as suggested by the ratio between main species and isotopomer. As already mentioned in the previous section, $\tau_{12}$, which is position-dependent and has 
Table 4. $\mathrm{CH}_{3} \mathrm{CN}$ and $\mathrm{CH}_{3}{ }^{13} \mathrm{CN}$ line parameters for $\mathrm{G} 31.41+0.31$.

\begin{tabular}{|c|c|c|c|c|c|}
\hline $\begin{array}{c}\text { Line } \\
K\end{array}$ & $\begin{array}{c}V_{\mathrm{LSR}} \\
\left(\mathrm{km} \mathrm{s}^{-1}\right)\end{array}$ & $\begin{array}{l}F W H M \\
\left(\mathrm{~km} \mathrm{~s}^{-1}\right)\end{array}$ & $\begin{array}{l}T_{\mathrm{B}}^{a} \\
(\mathrm{~K})\end{array}$ & $\begin{array}{c}\int T_{\mathrm{B}} \mathrm{d} V \\
\left(\mathrm{~K} \mathrm{~km} \mathrm{~s}^{-1}\right)\end{array}$ & $\begin{array}{c}\mathrm{rms} \\
\left(\mathrm{K} \mathrm{km} \mathrm{s}^{-1}\right)\end{array}$ \\
\hline & \multicolumn{5}{|c|}{$\mathrm{CH}_{3} \mathrm{CN}(5-4)$} \\
\hline 0 & $96.29 \pm 0.03$ & $9.77 \pm 0.04$ & $19.0 \pm 1.3$ & $198 \pm 3$ & 0.8 \\
\hline 1 & " & $"$ & $15.9 \pm 1.3$ & $165 \pm 3$ & $"$ \\
\hline 2 & $"$ & " & $21.6 \pm 1.3$ & $225 \pm 2$ & " \\
\hline 3 & $"$ & " & $22.2 \pm 1.3$ & $231 \pm 2$ & $"$ \\
\hline \multirow[t]{2}{*}{4} & $"$ & $"$ & $14.2 \pm 1.3$ & $148 \pm 2$ & $"$ \\
\hline & \multicolumn{5}{|c|}{$\mathrm{CH}_{3}{ }^{13} \mathrm{CN}(5-4)$} \\
\hline 0 & $97.41 \pm 0.03$ & $6.11 \pm 0.05$ & $5.83 \pm 0.46$ & $37.9 \pm 0.6$ & 0.24 \\
\hline 1 & " & " & $6.45 \pm 0.46$ & $41.9 \pm 0.6$ & $"$ \\
\hline 2 & $"$ & $"$ & $4.76 \pm 0.46$ & $31.0 \pm 0.6$ & $"$ \\
\hline 3 & $"$ & " & $5.41 \pm 0.46$ & $35.2 \pm 0.6$ & " \\
\hline \multirow[t]{2}{*}{4} & $97.41^{b}$ & $6.11^{b}$ & $2.17 \pm 0.18^{c}$ & $14.1 \pm 1.2^{d}$ & 0.08 \\
\hline & \multicolumn{5}{|c|}{$\mathrm{CH}_{3} \mathrm{CN}(12-11)$} \\
\hline 0 & $96.24 \pm 0.06$ & $8.07 \pm 0.08$ & $36.7 \pm 1.4$ & $315 \pm 8$ & 2.9 \\
\hline 1 & $"$ & $"$ & $31.3 \pm 1.4$ & $269 \pm 8$ & $"$ \\
\hline 2 & $"$ & $"$ & $39.0 \pm 1.4$ & $335 \pm 8$ & $"$ \\
\hline 3 & $"$ & $"$ & $40.4 \pm 1.4$ & $347 \pm 8$ & $"$ \\
\hline 4 & $96.84 \pm 0.09$ & $8.10 \pm 0.12$ & $34.9 \pm 1.2$ & $301 \pm 7$ & 2.5 \\
\hline 5 & $"$ & " & $26.9 \pm 1.2$ & $232 \pm 11$ & $"$ \\
\hline 6 & $96.78 \pm 0.16$ & $8.00^{b}$ & $24.3 \pm 3.0$ & $207 \pm 7$ & 1.5 \\
\hline 7 & $97.15 \pm 0.35$ & $8.00^{b}$ & $16.1 \pm 3.0$ & $137 \pm 16$ & 3.6 \\
\hline 8 & $"$ & $"$ & $10.9 \pm 3.0$ & $93 \pm 18$ & $"$ \\
\hline 9 & $97.85 \pm 1.28$ & $8.00^{b}$ & $e$ & $e$ & 3.4 \\
\hline 10 & $"$ & " & $3.4 \pm 0.7$ & $29 \pm 22$ & $"$ \\
\hline \multirow[t]{2}{*}{11} & $97.12 \pm 0.65$ & $8.00^{b}$ & $1.0 \pm 1.6$ & $8 \pm 17$ & 2.6 \\
\hline & \multicolumn{5}{|c|}{$\mathrm{CH}_{3}{ }^{13} \mathrm{CN}(12-11)$} \\
\hline 0 & $96.84 \pm 0.09$ & $8.10 \pm 0.12$ & $f$ & $f$ & 2.5 \\
\hline 1 & " & " & $14.2 \pm 2.5$ & $122 \pm 8$ & $"$ \\
\hline 2 & $"$ & $"$ & $12.9 \pm 2.5$ & $111 \pm 6$ & $"$ \\
\hline 3 & $96.78 \pm 0.16$ & $8.00^{b}$ & $19.8 \pm 3.0$ & $169 \pm 7$ & 1.5 \\
\hline 4 & $"$ & $"$ & $9.0 \pm 3.0$ & $76.3 \pm 6.6$ & $"$ \\
\hline 5 & " & " & $5.0 \pm 3.0^{g}$ & $42.2 \pm 7.2^{g}$ & $"$ \\
\hline 6 & $"$ & $"$ & $3.8 \pm 3.0$ & $32.1 \pm 6.6$ & $"$ \\
\hline 7 & $97.85 \pm 1.28$ & $8.00^{b}$ & $2.6 \pm 0.7$ & $22 \pm 21$ & 3.4 \\
\hline 8 & $97.12 \pm 0.65$ & $8.00^{b}$ & $15.0 \pm 1.6^{h}$ & $127 \pm 17^{h}$ & 2.6 \\
\hline 9 & $"$ & $"$ & $6.4 \pm 1.6$ & $55 \pm 17$ & . \\
\hline
\end{tabular}

${ }^{a}$ Brightness temperature integrated over a $3 \sigma$ emission level area.

${ }^{b}$ This parameter was held fixed in the fit.

${ }^{c}$ Computed from the integrated line intensity.

${ }^{d}$ Measured with the command print area of CLASS.

${ }^{e}$ Blended with ${ }^{13} \mathrm{CO}(2-1)$.

${ }^{f}$ Blended with $\mathrm{CH}_{3} \mathrm{CN} K=5$.

${ }^{g}$ Blended with $\mathrm{CH}_{3} \mathrm{CN} K=7$.

${ }^{h}$ Dominated by wing emission of ${ }^{13} \mathrm{CO}(2-1)$.

been evaluated at the systemic velocity, ranges from 10 to 50 for $K=2$, and from 5 to 20 for $K=6$ in the core. Thus, in the fit performed to the Boltzmann plot we used only the transitions of $\mathrm{CH}_{3}{ }^{13} \mathrm{CN}$ and those from the $\mathrm{CH}_{3} \mathrm{CN} v_{8}=1$ state (see Fig. 6). All the spectra were integrated over a region of $\sim 4$. $7^{\prime \prime}$ in diameter $\left(\sim 17 \operatorname{arcsec}^{2}\right)$, in order to include all the emission inside the $3 \sigma$ contour level for the (5-4) transition. Assuming that also the emission due to the vibrational state is optically thin and a relative abundance $\left[\mathrm{CH}_{3} \mathrm{CN}\right] /\left[\mathrm{CH}_{3}{ }^{13} \mathrm{CN}\right]=50$ (see Wilson \& Rood 1994), the $T_{\text {rot }}$ and source averaged $N_{\mathrm{CH}_{3} \mathrm{CN}}$ derived by taking into account all transitions mentioned above are $164 \pm 5 \mathrm{~K}$ and $8 \times 10^{16} \mathrm{~cm}^{-2}$, respectively. We also estimated $T_{\text {rot }}$ and $N_{\mathrm{CH}_{3} \mathrm{CN}}$ from each set of $J+1 \rightarrow J$ lines separately. The rotation temperatures estimates are $135 \pm 2$ and $220 \pm 16 \mathrm{~K}$, 
Table 5. $\mathrm{CH}_{3} \mathrm{CN}(5-4) v_{8}=1$ line parameters for $\mathrm{G} 31.41+0.31$.

\begin{tabular}{lccccc}
\hline \hline Line & $\begin{array}{c}V_{\mathrm{LSR}} \\
\left(\mathrm{km} \mathrm{s}^{-1}\right)\end{array}$ & $\begin{array}{c}F W H M \\
\left(\mathrm{~km} \mathrm{~s}^{-1}\right)\end{array}$ & $\begin{array}{c}T_{\mathrm{B}}^{a} \\
(\mathrm{~K})\end{array}$ & $\begin{array}{c}\int T_{\mathrm{B}} \mathrm{d} V \\
\left(\mathrm{~K} \mathrm{~km} \mathrm{~s}^{-1}\right)\end{array}$ & $\begin{array}{c}\mathrm{rms} \\
\left(\mathrm{K} \mathrm{km} \mathrm{s}^{-1}\right)\end{array}$ \\
\hline$(1,1)$ & $97.45 \pm 0.41$ & $8.02 \pm 0.44$ & $5.15 \pm 0.24$ & $44 \pm 5$ & 1.0 \\
$(2,1)$ & $"$ & $"$ & $4.69 \pm 0.24$ & $40 \pm 5$ & $"$ \\
$(0,1)$ & $"$ & $"$ & $4.62 \pm 0.24$ & $39 \pm 5$ & $"$ \\
$(3,1)$ & $"$ & $"$ & $3.65 \pm 0.24$ & $31 \pm 5$ & $"$ \\
$(1,-1)$ & $"$ & $"$ & $4.12 \pm 0.24$ & $35 \pm 5$ & $"$ \\
$(4,1)$ & $97.45 \pm 0.50$ & $8.71 \pm 0.69$ & $3.17 \pm 0.31$ & $29 \pm 7$ & 1.2 \\
$(2,-1)$ & $"$ & $"$ & $4.95 \pm 0.31$ & $46 \pm 7$ & $"$ \\
$(3,-1)$ & $"$ & $"$ & $3.27 \pm 0.31$ & $30 \pm 7$ & $"$ \\
$(4,-1)$ & $"$ & $"$ & $1.63 \pm 0.31$ & $15 \pm 6$ & $"$ \\
$(1,1)$ & $97.45^{b}$ & $8.71^{b}$ & $4.72 \pm 0.12^{c}$ & $39 \pm 1^{d}$ & 0.09 \\
\hline
\end{tabular}

${ }^{a}$ Brightness temperature integrated over a $3 \sigma$ emission level area.

${ }^{b}$ This parameter was held fixed in the fit.

${ }^{c}$ Computed from the integrated line intensity.

${ }^{d}$ Measured with the command print area of CLASS.

and the column densities $9 \times 10^{16}$ and $7 \times 10^{16} \mathrm{~cm}^{-2}$ for the (5-4) and (12-11) transitions, respectively. These values of $T_{\text {rot }}$ are consistent with those derived from $\mathrm{CH}_{3} \mathrm{CN}$ and $\mathrm{CH}_{3}{ }^{13} \mathrm{CN}$ (6-5) by Olmi et al. (1996a) for the compact component of the molecular clump $\left(\sim 1-2^{\prime \prime}\right)$, called "core", and the most extended one $\left(\sim 10-20^{\prime \prime}\right)$, called "halo", which are 230 and $130 \mathrm{~K}$, respectively. The fact that the $T_{\text {rot }}$ that we derived by using only the (12-11) transitions is close to the value derived by Olmi et al. (1996a) for the "core", and that derived using only the (5-4) lines is closer to the value for the "halo", could be indicating that the highest excitation transitions are tracing material closer to the embedded source, while the lowest excitation transitions are tracing material in the outer shell of the core. From this analysis, it is clear that the temperatures in $\mathrm{G} 31.41+0.31$ are very high, confirming that the methyl cyanide emission comes from hot molecular gas. Regarding the column densities, the estimates of the order of $\sim 10^{17} \mathrm{~cm}^{-2}$ are consistent with those obtained by Olmi et al. (1996a,b).

In order to establish the existence of a temperature gradient in the core, as suggested by Figs. 3 and 5 (see Sect. 3.1.2), we computed a map of $T_{\text {rot }}$ by fitting the Boltzman plots at each position (pixel) in the core. The fits were performed with the RD method to the $\mathrm{CH}_{3}{ }^{13} \mathrm{CN} K=1,2,3,4,5$, and 6 components $(K=0$ was not used because too blended with $\mathrm{CH}_{3} \mathrm{CN} K=5$ ). Figure 7 shows the resulting map of $T_{\text {rot }}$, as well as the total column density map, $N_{\mathrm{CH}_{3} \mathrm{CN}}$, derived with the same method; for the sake of comparison the $\mathrm{CH}_{3}{ }^{13} \mathrm{CN}$ (12-11) emission averaged under the $K=1$ and 2 components is also shown. This figure clearly confirms that the temperatures reached toward the center of the core, where the millimeter continuum source is embedded and the lower $K$-components methyl cyanide emission shows a dip, are very high $(\sim 350 \mathrm{~K})$, while the temperatures toward the peak of $\mathrm{CH}_{3}{ }^{13} \mathrm{CN}$ are lower $(\sim 200-250 \mathrm{~K})$. The temperature at the position of the peaks of the torus seen in the $\mathrm{CH}_{3} \mathrm{CN}$ emission (see Fig. 5) is even lower, $T_{\text {rot }} \sim 150 \mathrm{~K}$. For this source Cesaroni et al. (1998) have derived a temperature gradient of the type $T \propto R^{-3 / 4}$ using
$\mathrm{NH}_{3}(4,4)$ : this is consistent with our findings $350 \sim 150 \times 3^{3 / 4}$, where 3 is roughly the value of the distance where the temperature is $150 \mathrm{~K}$ divided by the distance where the temperature is $350 \mathrm{~K}$. The $N_{\mathrm{CH}_{3} \mathrm{CN}}$ map also shows that the gas column density increases toward the center, with the peak at the position of the millimeter continuum source, confirming that the $\mathrm{CH}_{3} \mathrm{CN}$ gas is present also in the central region of the core.

The geometry of the G31.41+0.31 core seems to be the result of the combination of two effects: the existence of a temperature gradient in the core plus opacity. In Sect. 3.1.2 we checked for a possible opacity effect by computing the optical depth at each point assuming, as a first approximation, a single excitation temperature. However, because of the existence of a temperature gradient in the core, the optical depth can be better measured taking it into account. In order to better fit the data and understand the geometry of the core we computed models which solve the radiative transfer equation. These models are discussed in the next section.

\subsubsection{Velocity field: The rotating toroid}

In Paper I we detected a clear velocity gradient in the G31.41+0.31 core by simultaneously fitting multiple $\mathrm{CH}_{3} \mathrm{CN}$ (12-11) $K$-components at each position where $\mathrm{CH}_{3} \mathrm{CN}$ is detected. This velocity gradient is perpendicular to the molecular outflow detected in the region by Olmi et al. (1996a), and as already discussed in Paper I, the most plausible explanation for such a velocity gradient is that the core is undergoing rotation about the outflow axis. Also Gibb et al. (2004) have studied the velocity field in $\mathrm{G} 31.41+0.31$ by analyzing the $\mathrm{C}^{18} \mathrm{O}$ and $\mathrm{H}_{2} \mathrm{~S}$ line emission. By mapping the $\mathrm{H}_{2} \mathrm{~S}$ line wings, these authors have detected a velocity gradient in the same direction as our $\mathrm{CH}_{3} \mathrm{CN}$ gradient, but have interpreted this as a bipolar outflow, with the blueshifted emission toward the west and the redshifted toward the east. To our knowledge a high-density tracer such as $\mathrm{CH}_{3} \mathrm{CN}$ has never been found to trace outflows: instead there are clear examples of $\mathrm{CH}_{3} \mathrm{CN}$ emission tracing 


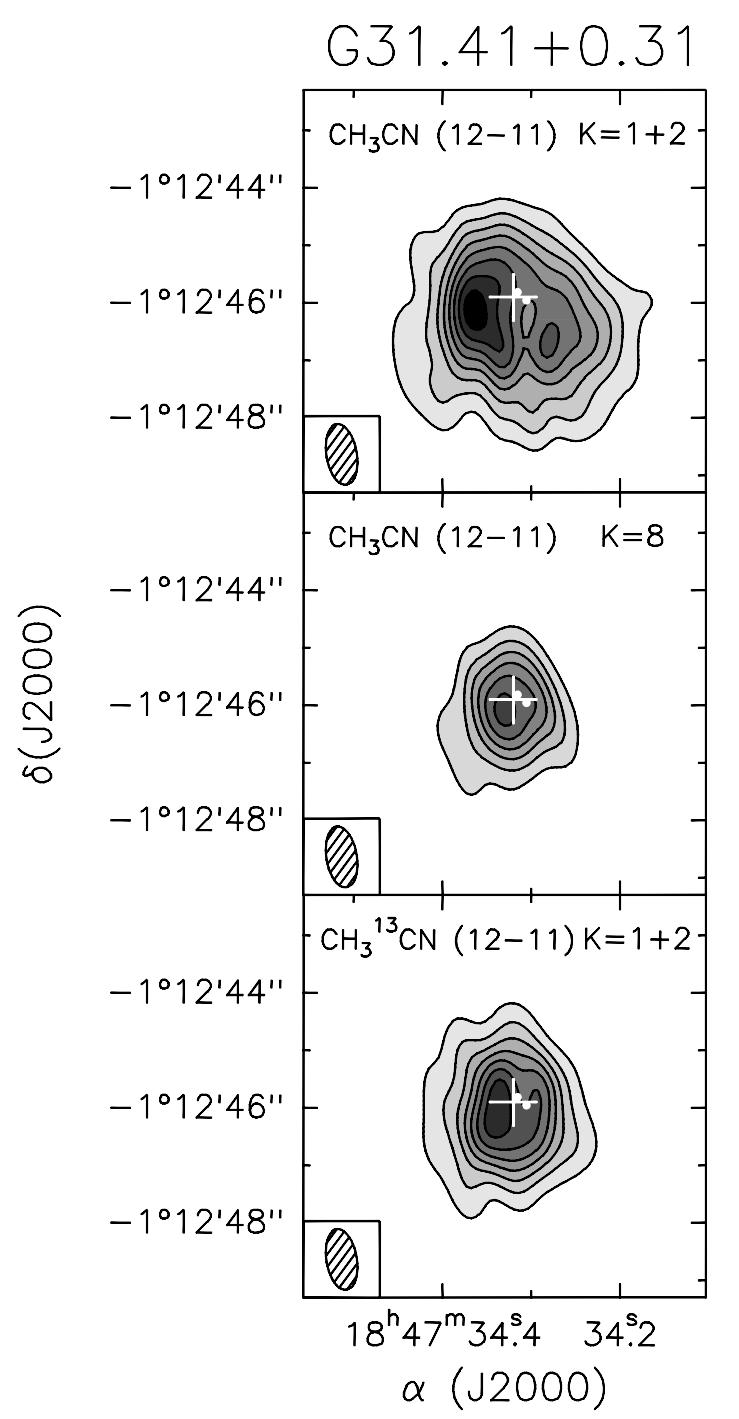

Fig. 3. $\mathrm{PdBI}$ intensity maps of the $\mathrm{CH}_{3} \mathrm{CN}(12-11)$ emission averaged under the $K=1$ and 2 components (top panel), under the $K=8$ component (middle panel), and of the $\mathrm{CH}_{3}{ }^{13} \mathrm{CN}$ (12-11) emission averaged under the $K=1$ and 2 components (bottom panel) toward $\mathrm{G} 31.41+0.31$. The contour levels range from 0.1 to $0.94 \mathrm{Jy}^{\mathrm{beam}}{ }^{-1}$ (top panel), to $0.70 \mathrm{Jy}^{\text {beam }}{ }^{-1}$ (middle panel), and to $0.82 \mathrm{Jy} \mathrm{beam}^{-1}$ (bottom panel), in steps of $0.12 \mathrm{Jy}^{\text {beam }^{-1}}$. The synthesized beam is shown in the lower left-hand corner. The white cross marks the position of the $1.4 \mathrm{~mm}$ continuum emission peak, and the white dots the $7 \mathrm{~mm}$ continuum emission peaks detected by Hofner (private communication).

rotating disks, for example IRAS 20126+4104 (Cesaroni et al. 1999) or G24.78+0.08 (Paper I); in the latter the velocity gradient seen in $\mathrm{CH}_{3} \mathrm{CN}$ in core $\mathrm{A} 1$ is clearly perpendicular to the bipolar outflow mapped in ${ }^{12} \mathrm{CO}$ (Furuya et al.2002). We hence believe that the interpretation given in Paper I for the $\mathrm{CH}_{3} \mathrm{CN}$ emission in $\mathrm{G} 31.41+0.31$ is to be preferred to that of Gibb et al. (2004), and consider the velocity gradient detected a signature of rotation. Only high-angular resolution observations in unambiguous outflow tracers such as ${ }^{12} \mathrm{CO}$ may definitely prove which interpretation is correct.

The velocity shift measured over an extent of $\sim 17000 \mathrm{AU}$ is $4.2 \mathrm{~km} \mathrm{~s}^{-1}$ (Paper I), which corresponds to a velocity

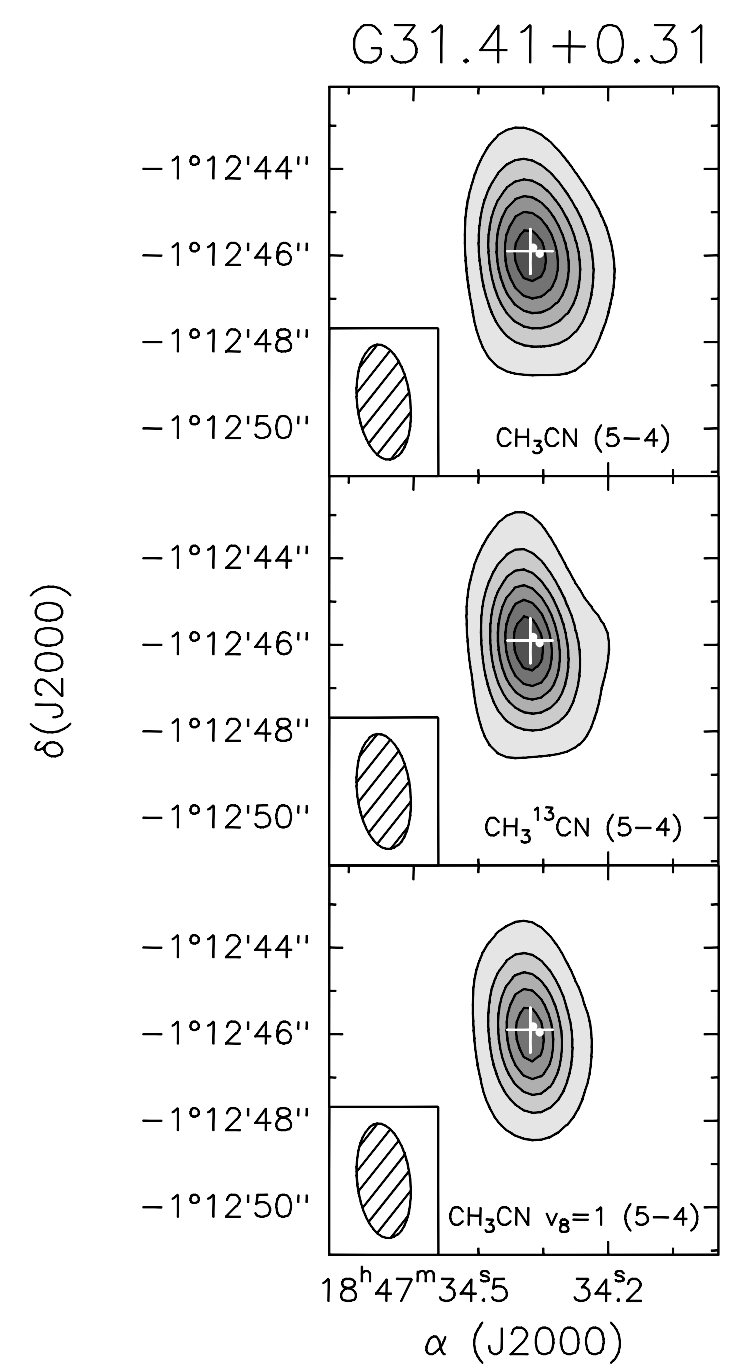

Fig. 4. $\mathrm{PdBI}$ intensity maps of the $\mathrm{CH}_{3} \mathrm{CN}$ (5-4) (top panel) and the $\mathrm{CH}_{3}{ }^{13} \mathrm{CN}$ (middle panel) emission averaged under the $K=0$ to 4 components, and of the $v_{8}=1$ emission (bottom panel) averaged under the $(1,1),(2,1),(0,1),(3,1),(1,-1),(4,1),(2,-1),(3,-1),(4,-1)$, and $(1,1)$ transitions toward G31.41+0.31. The contour levels range from 0.1 to $0.70 \mathrm{Jy}$ beam $^{-1}$ in steps of $0.12 \mathrm{Jy} \mathrm{beam}^{-1}$ (top panel), from 0.02 to $0.22 \mathrm{Jy} \mathrm{beam}^{-1}$ (middle panel), and to $0.18 \mathrm{Jy}$ beam $^{-1}$ (bottom panel) in steps of $0.04 \mathrm{Jy}_{\text {beam }}{ }^{-1}$. The synthesized beam is shown in the lower left-hand corner. The white cross marks the position of the $1.4 \mathrm{~mm}$ continuum emission peak, and the white dots the $7 \mathrm{~mm}$ continuum emission peaks detected by Hofner (private communication).

gradient of about $50 \mathrm{~km} \mathrm{~s}^{-1} \mathrm{pc}^{-1}$. The size and the mass of $\sim 500 M_{\odot}$ suggest that this toroid could be hosting not a single massive YSO but a cluster of YSOs. This is also suggested by the continuum emission at $7 \mathrm{~mm}$ toward the center, which reveals two point-like sources oriented in the NE-SW direction (Hofner, private communication), i.e. just along the toroid plane. It is hence tempting to speculate that collapse in the toroid has led to their formation.

The kinematics of the gas toward the core of G31.41+0.31 can be seen in the position-velocity (PV) cut of the $\mathrm{CH}_{3} \mathrm{CN}$ (12-11) $K=3$ emission along a direction (PA $\sim 70^{\circ}$ ) where the velocity gradient is maximum (Fig. 8). Clearly, Keplerian rotation, which has been detected toward lower luminous 


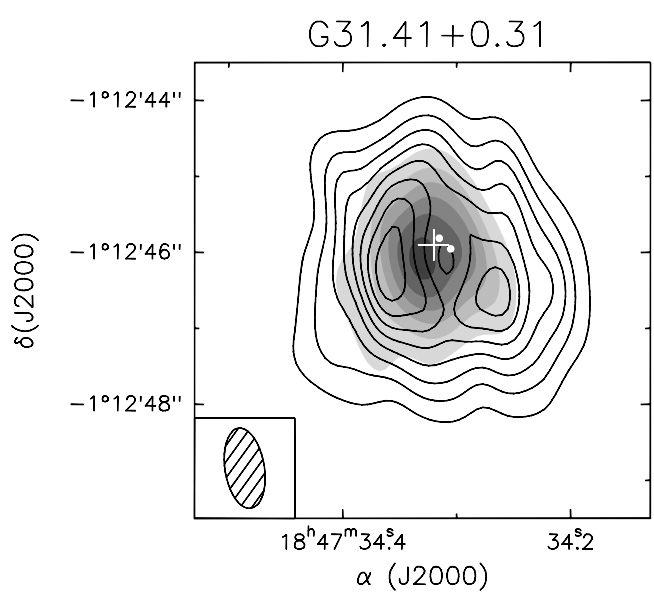

Fig. 5. Overlay of the $\mathrm{PdBI} \mathrm{CH}_{3} \mathrm{CN}$ (12-11) emission averaged under the $K=0,1$, and 2 components (contours) on the emission under $K=8($ greyscale $)$ toward G31.41+0.31. Contour levels range from 0.1 to $0.94 \mathrm{Jy}_{\text {beam }}{ }^{-1}$ in steps of $0.12 \mathrm{Jy}_{\text {beam }}{ }^{-1}$. Greyscale levels range from 0.1 to $0.70 \mathrm{Jy} \mathrm{beam}^{-1}$ in steps of $0.12 \mathrm{Jy} \mathrm{beam}^{-1} \mathrm{~km} \mathrm{~s}^{-1}$. The synthesized beam is shown in the lower left-hand corner. The white cross marks the position of the $1.4 \mathrm{~mm}$ continuum emission peak, and the white dots the $7 \mathrm{~mm}$ continuum emission peaks detected by Hofner (private communication).

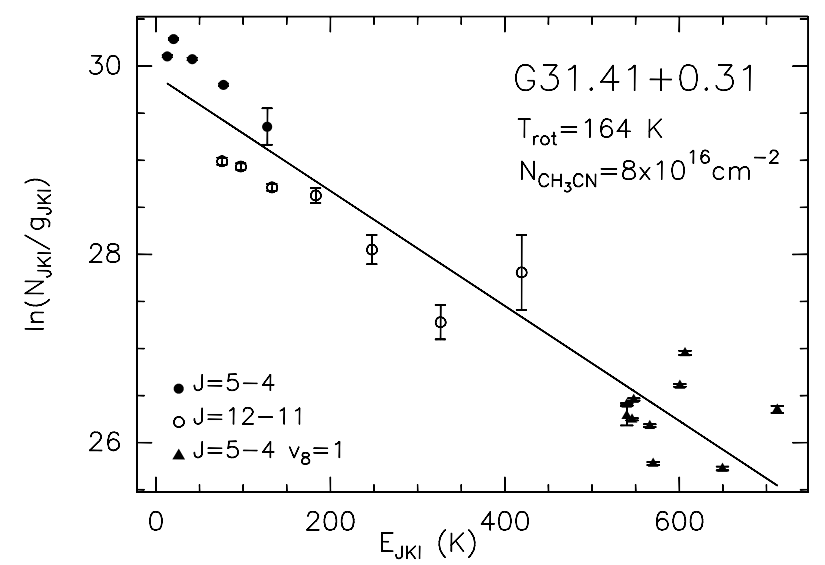

Fig. 6. Rotation diagram for G31.41+0.31 with superimposed fit. Filled circles, open circles, and filled triangles correspond to the $\mathrm{CH}_{3}{ }^{13} \mathrm{CN}$ (5-4), the $\mathrm{CH}_{3}{ }^{13} \mathrm{CN}$ (12-11), and the $\mathrm{CH}_{3} \mathrm{CN}$ (5-4) $v_{8}=1$ transition, respectively.

objects such as IRAS 20126+4104 (Cesaroni et al. 1997, 1999; Zhang et al. 1998), NGC 7538S (Sandell et al. 2003), or M17 (Chini et al. 2004), is not possible as the mass of the toroid is much larger than any reasonable stellar mass. In fact, the PV cut shows no hint of Keplerian rotation. However, it is interesting to see that according to theoretical models (Galli, private communication), discriminating between a velocity field with constant rotation velocity or with constant angular velocity should help to assess whether magnetic braking, and thus magnetic field, plays a crucial role during the process of disk, and hence star, formation. In the absence of magnetic field, a self-gravitating isothermal quasistatic equilibrium situation is reached when the rotation velocity is constant, $v=$ constant $(\gamma=0)$. When a magnetic field is present, it couples the core

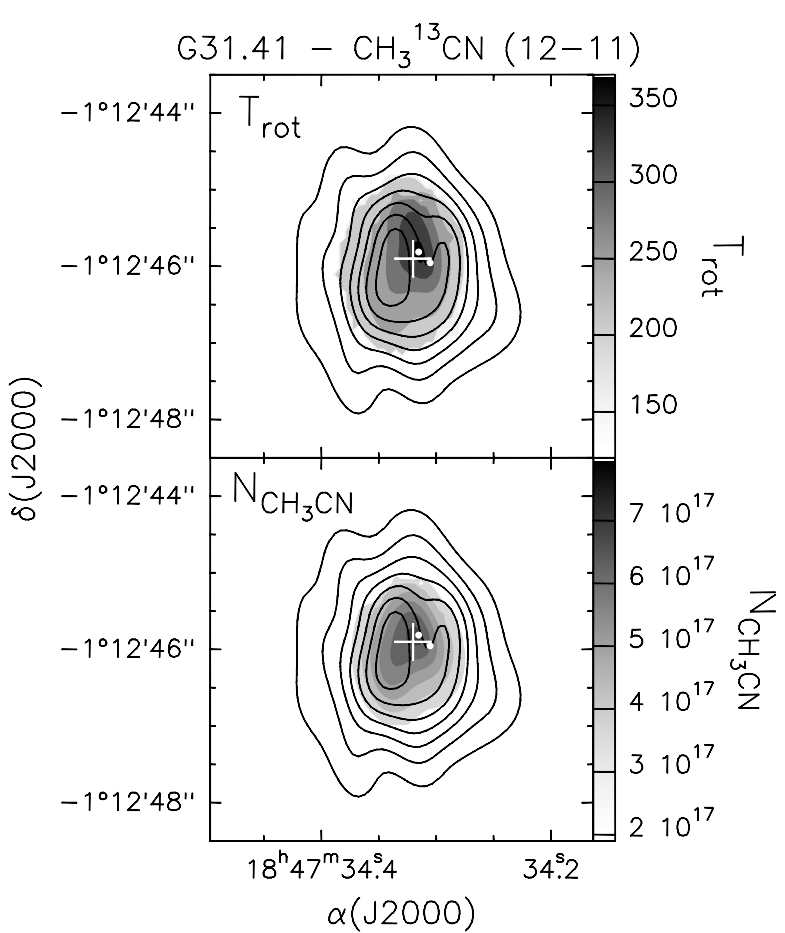

Fig. 7. Overlay of the $\mathrm{PdBI} \mathrm{CH}{ }_{3}{ }^{13} \mathrm{CN}(12-11)$ emission averaged under the $K=1$ and 2 components (contours) on the $T_{\text {rot }}$ map (top panel) and the $N_{\mathrm{CH}_{3} \mathrm{CN}}$ map (bottom panel) in greyscale, derived by fitting the Boltzmann plots at each position, toward G31.41+0.31. The contour levels range from 0.1 to $0.82 \mathrm{Jy}^{\mathrm{beam}^{-1}}$ in steps of $0.12 \mathrm{Jy}_{\text {beam }}^{-1}$. Greyscale levels range from 100 to $350 \mathrm{~K}$ by $50 \mathrm{~K}$ (top panel), and from 2 to $7 \times 10^{17} \mathrm{~cm}^{-2}$ by $1 \times 10^{17} \mathrm{~cm}^{-2}$ (bottom panel). The white cross marks the position of the $1.4 \mathrm{~mm}$ continuum emission peak, and the white dots the $7 \mathrm{~mm}$ continuum emission peaks detected by Hofner (private communication).

with the surrounding medium by means of magnetic braking. This coupling yields a constant angular velocity, $v \propto R(\gamma=1)$, in the whole core, which rotates as a rigid body and leads by ambipolar diffusion to an equilibrium situation. Thus, in order to discriminate between the two possible scenarios, which could give an indication of the stability of the cores and a hint of their evolutionary stage, we modeled the $\mathrm{CH}_{3} \mathrm{CN}$ emission by assuming that the emission arises from a disk-like structure seen edge-on by the observer with an internal radial velocity field $\left(v \propto R^{\gamma}\right)$; a constant rotation velocity field and a constant angular velocity field.

The models were computed by adopting a power law dependence on the distance from the core center also for the temperature $T \propto R^{-q}$ of the emitting gas. According to accretion disk theory for geometrically thin disks the dependence of the temperature on the radius is $T \propto R^{-3 / 4}$ (see Natta 2000, and references therein). Although geometrically thick, these toroids resemble more 2D structures like circumstellar disks than 3D spherically symmetric cores. Therefore, we have adopted a temperature profile $T \propto R^{-3 / 4}$ in our models. This is also indicated by the findings of Cesaroni et al. (1998), who have properly modeled the $\mathrm{NH}_{3}(4,4)$ emission toward $\mathrm{G} 31.41+0.31$ with $T \propto R^{-3 / 4}$. For simplicity we adopted a constant density in the 


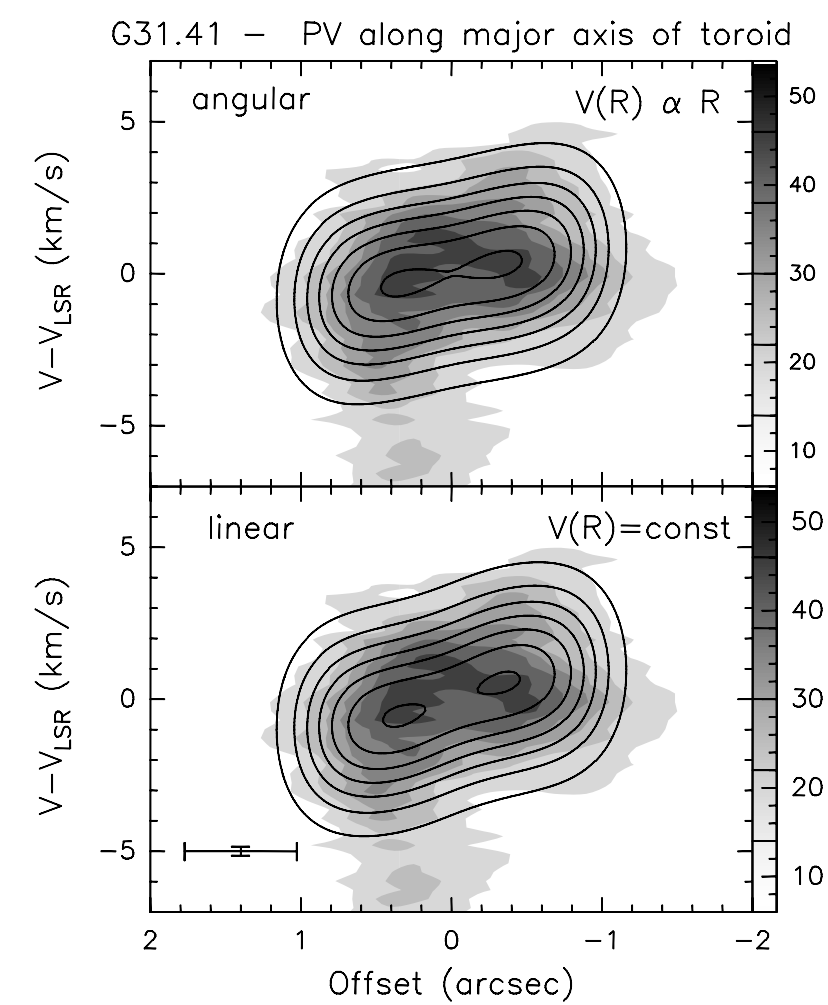

Fig. 8. Overlay of the PV plot of the $\mathrm{CH}_{3} \mathrm{CN}(12-11) K=3$ emission (greyscale) along the major axis of the toroid $\left(\mathrm{PA} \sim 70^{\circ}\right)$ in $\mathrm{G} 31.41+0.31$, and the synthetic emission PV plot of a model (contours) with constant angular velocity (top panel), and with constant rotation velocity (bottom panel). The $y$-axis plots the difference between the measured velocity and the systemic velocity, which is $97 \mathrm{~km} \mathrm{~s}^{-1}$ for $\mathrm{G} 31.41+0.31$. The contour levels range from 6 to $54 \mathrm{Jy} \mathrm{beam}^{-1}$ in steps of $8 \mathrm{Jy} \mathrm{beam}^{-1}$. The error bars in the lower left-hand corner indicate the angular and spectral resolution of the data and the models.

core. The synthetic PV diagrams were computed along the projected major axis of the toroid, and convolved with a Gaussian in the PV plane to obtain the same angular and spectral resolutions as those of the data. The parameters of the models are the inner and outer radius of the toroid, $R_{\text {inn }}$ and $R_{\text {out }}$, respectively, a parameter related to the column density at the peak of the emission, the observed line width, $\Delta V$, the power-law index $p$ of the density, the temperature at the outer radius, $T_{\text {out }}$, the power-law index $q$ of the temperature, and the rotation velocity measured at the edge of the toroid, $v_{\text {rot }}$. The initial guesses for the input parameters such as the size of the toroid or the rotation velocity were derived from the observations (see Paper I).

The observed and synthetic PV diagrams for both scenarios, constant angular velocity (angular) and constant rotation velocity (linear), can be seen in Fig. 8. From the modeling itself it is not possible to distinguish between constant angular ( $v \propto R$; top panel) or constant rotation velocity $(v=$ constant; bottom panel), because both velocity fields suitably fit the data. The models were computed with and without temperature gradients. However, only those models with $T \propto R^{-3 / 4}$ can fit properly the observed PV plots and reproduce the "inner hole" seen in the data. A similar result could be also obtained with a bigger $R_{\text {inn }}$. However, in this case the hole at the center of the toroid would be also visible in the millimeter continuum emission maps. The best fit model parameters are given in Table 6. The value of $R_{\text {inn }}$ is $0{ }^{\prime} 17$ (1340 AU), which is roughly the separation between the two sources detected at $7 \mathrm{~mm}$ (Hofner, private communication). The values of $v_{\text {rot }}$ and $R_{\text {out }}$ are consistent with those derived directly from the velocity gradient and the $1.4 \mathrm{~mm}$ continuum emission at $50 \%$ of the peak, respectively (see Table 1 in Paper I). The value of the column density at the peak of the $\mathrm{CH}_{3} \mathrm{CN}$ emission, $N_{\mathrm{CH}_{3} \mathrm{CN}}^{\text {peak }}$, is $\sim 20$ times lower than the column density derived by means of the RD method (see previous section). This is due to the fact that the models do not take into account the clumpiness of the region (i.e. a beam filling factor effect). The models also support the scenario according to which the lack of emission at the center of the toroid is not a geometric effect but is due to selfabsorption, which is caused by the high temperatures and optical depths reached toward the center of the core, as suggested by the $\mathrm{CH}_{3} \mathrm{CN}$ and $\mathrm{CH}_{3}{ }^{13} \mathrm{CN}$ integrated emission plots and by the column density distribution obtained from the Boltzman plots (see Sects. 3.1.2 and 3.2.1). In fact, optically thin models with a temperature gradient cannot fit the data because in such a case, the external layers of the toroid become too optically thin to absorb enough photons from the center to reproduce the lack of emission or self-absorption visible in $\mathrm{CH}_{3} \mathrm{CN}$. Thus, the models indicate that the emission in $\mathrm{G} 31.41+0.31$ has to be optically thick and that a temperature gradient is present in the core.

The dynamical mass needed for equilibrium, $M_{\mathrm{dyn}}$, has been derived from the expression $M_{\mathrm{dyn}}=v_{\mathrm{rot}}^{2} R_{\mathrm{out}} / G \sin ^{2} i$, where $i$ is the inclination angle assumed to be $90^{\circ}$ for an edgeon toroid. Note that this expression in Paper I is wrong: $\sin ^{2} i$ should be dividing, but the values of $M_{\text {dyn }}$ given in Table 1 were correctly computed. The value obtained is $M_{\text {dyn }} \sim 44 M_{\odot}$. The fact that $M_{\mathrm{dyn}}$ is much smaller than the mass of the core suggests, as already pointed out in Paper I, that such structure is unstable against gravitational collapse. Note that the magnetic field required to stabilize it would be of a few $20-40 \mathrm{mG}$, values too large to be plausible even in regions as dense as $10^{8} \mathrm{~cm}^{-3}$ (see Fig. 1 of Crutcher 1999).

In case that the toroid is undergoing collapse the gas should be more perturbed toward the center, where the $1.4 \mathrm{~mm}$ continuum source is embedded, and the velocity dispersion should increase inward. Thus, one would expect the line width, $\Delta V$, to increase toward the center of the toroid. Indeed Fig. 9 shows that $\Delta V$, which has been obtained by fitting the optically thin $\mathrm{CH}_{3}{ }^{13} \mathrm{CN}$ (12-11) line profiles at each position with a Gaussian, increases toward the center of the core, where the YSO(s) is embedded. One can obtain an estimate of the infall velocity, $v_{\text {inf }}$, assuming that the only contribution to the variation of $\Delta V$ is infall and that the contribution of turbulence is negligible. In such a case, $v_{\text {inf }}$ is given by the difference between $\Delta V$ at the position of the $1.4 \mathrm{~mm}$ continuum source and that at the edge of the toroid, $v_{\text {inf }} \simeq\left(\Delta V_{\text {inn }}-\Delta V_{\text {out }}\right) / 2$. Interestingly, the value obtained is $v_{\text {inf }} \simeq 2 \mathrm{~km} \mathrm{~s}^{-1}$, consistent with the $v_{\text {rot }}$ obtained from the models. Such agreement between $v_{\text {inf }}$ and $v_{\text {rot }}$ has been predicted by some recent theoretical studies (see e.g. Allen et al. 2003). Following the expression given in Paper I we estimated the accretion rate and obtained $\dot{M}_{\text {acc }} \sim 3 \times 10^{-2} M_{\odot} \mathrm{yr}^{-1}$. Accretion rates that large have been 
Table 6. Best fit model parameters for G31.41+0.31 and core A1 in G24.78+0.08.

\begin{tabular}{|c|c|c|c|c|c|c|c|c|c|}
\hline \multirow[b]{2}{*}{ Core } & \multicolumn{2}{|c|}{$R_{\text {inn }}$} & \multicolumn{2}{|c|}{$R_{\text {out }}$} & \multirow{2}{*}{$\begin{array}{l}T_{\text {out }} \\
(\mathrm{K})\end{array}$} & \multirow{2}{*}{$\begin{array}{c}N_{\mathrm{CH}_{3} \mathrm{CN}}^{\text {peak }} \\
\left(\mathrm{cm}^{-2}\right)\end{array}$} & \multirow{2}{*}{$\begin{array}{c}v_{\text {rot }} \\
\left(\mathrm{km} \mathrm{s}^{-1}\right)\end{array}$} & \multirow{2}{*}{$\begin{array}{c}\Delta V \\
\left(\mathrm{~km} \mathrm{~s}^{-1}\right)\end{array}$} & \multirow[t]{2}{*}{$q$} \\
\hline & $\left({ }^{\prime \prime}\right)$ & $(\mathrm{AU})$ & $\left({ }^{\prime \prime}\right)$ & $(\mathrm{AU})$ & & & & & \\
\hline G31 & 0.17 & 1340 & 1.7 & 13400 & 100 & $5 \times 10^{15}$ & 1.7 & 6.8 & -0.75 \\
\hline G24 A1 & 0.30 & 2300 & 1.0 & 7700 & 100 & $5 \times 10^{15}$ & 2.0 & 5.6 & -0.75 \\
\hline
\end{tabular}

${ }^{a}$ Column density at the peak of the $\mathrm{CH}_{3} \mathrm{CN}$ emission.

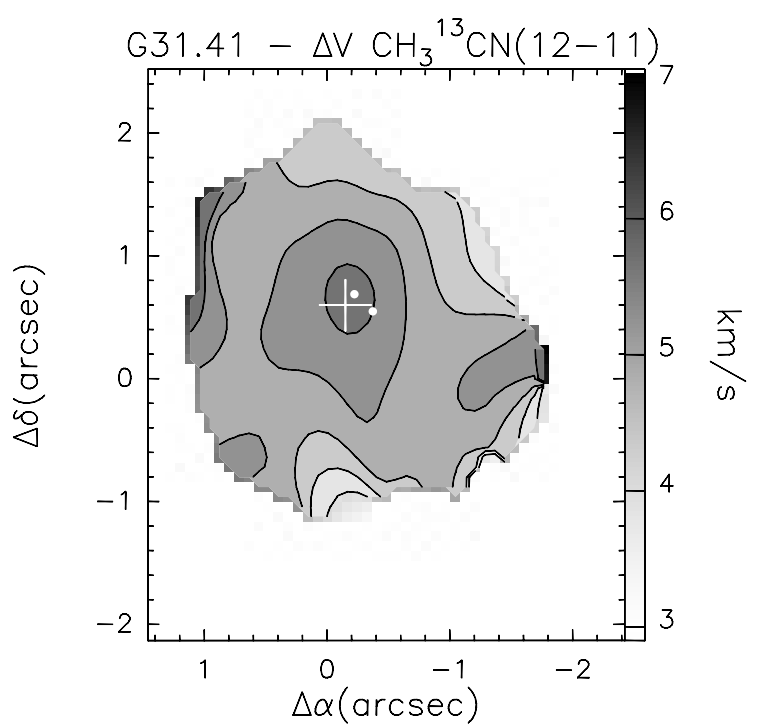

Fig. 9. Line width plot of $\mathrm{CH}_{3}{ }^{13} \mathrm{CN}(12-11)$ toward $\mathrm{G} 31.41+0.31$. The contour levels range from 3 to $7 \mathrm{~km} \mathrm{~s}^{-1}$ in steps of $1 \mathrm{~km} \mathrm{~s}^{-1}$. The white cross marks the position of the $1.4 \mathrm{~mm}$ continuum emission peak, and the white dots the $7 \mathrm{~mm}$ continuum emission peaks detected by Hofner (private communication).

estimated for the massive Class 0 object IRAS 23385+6053 (Molinari et al. 1998), and for the parsec-scale clumps in which high-mass star formation is observed (Fontani et al. 2002), and could support the theories that predict that high-mass stars might form through non-spherical accretion with large accretion rates.

\section{4. $\mathrm{G} 24.78+0.08$}

$\mathrm{G} 24.78+0.08$ is a high-mass star forming region that contains a cluster of massive YSOs in different evolutionary stages. Furuya et al. (2002) firstly detected four molecular cores in the region, identified as A, B, C, and D in Fig. 10, with two of them, core A and C, powering a bipolar molecular outflow. When observed with higher angular resolution, the continuum and methyl cyanide emission toward core $\mathrm{A}$ is resolved into two separate cores, named A1 and A2 in Paper I. Although one cannot rule out the possibility that the continuum and methyl cyanide peaks in A2 could be the result of the interaction of the outflow with the surrounding dense gas, we consider them as two distinct cores in our study (see a more detailed discussion in Sects. 4.2.2 and 4.2.3).

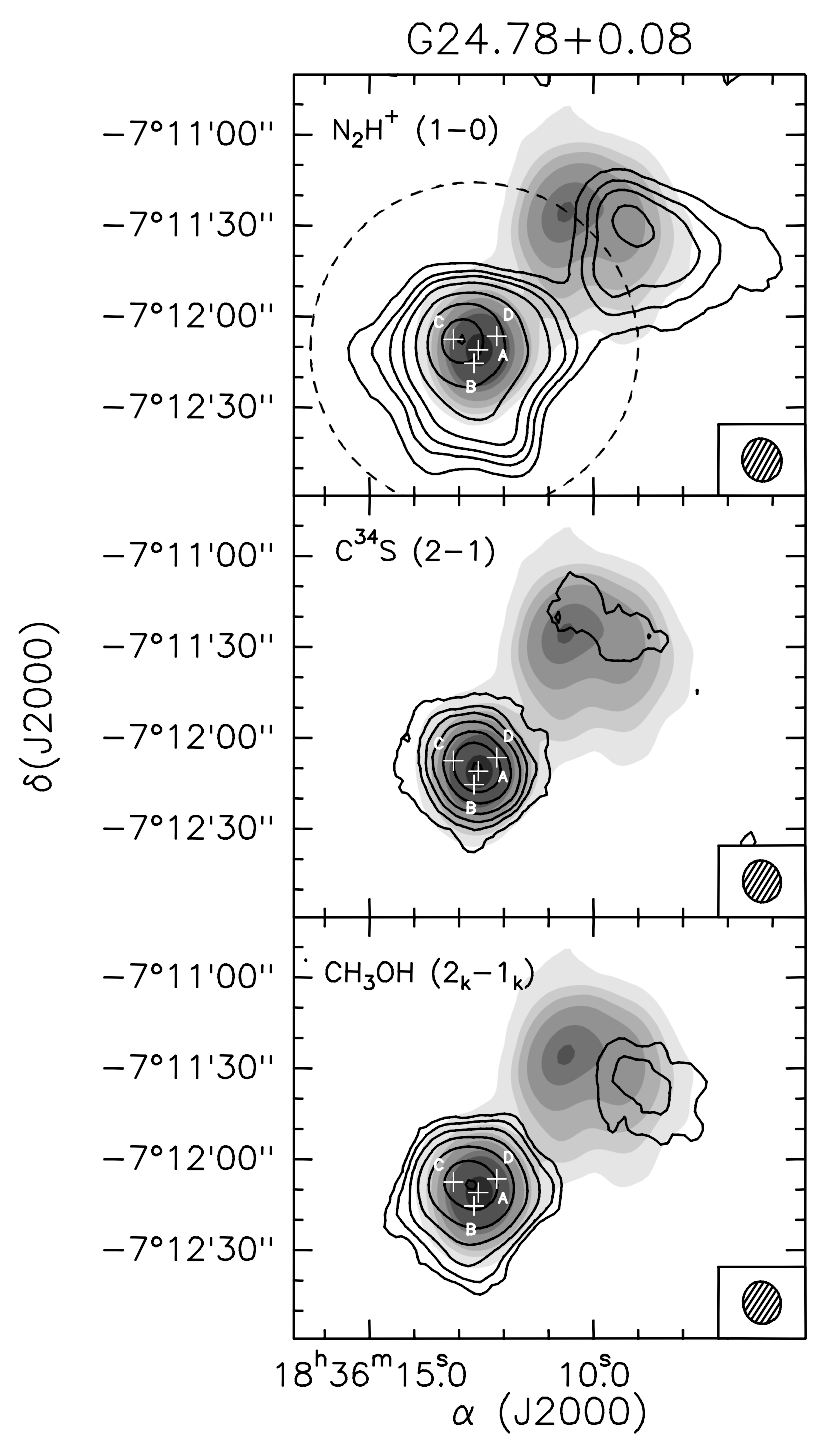

Fig. 10. Overlay of the BIMA integrated intensity maps of the $\mathrm{N}_{2} \mathrm{H}^{+}$ (1-0) emission (contours; top panel), the $\mathrm{C}^{34} \mathrm{~S}(2-1)$ emission (contours; middle panel), and the $\mathrm{CH}_{3} \mathrm{OH}\left(2_{k}-1_{k}\right)$ emission (contours; bottom panel) on the BIMA $3.16 \mathrm{~mm}$ continuum emission (greyscale) toward G24.78+0.08. The contours are 3, 9, 15, 25, 45, 75, 120, and $150 \mathrm{Jy} \mathrm{beam}^{-1} \mathrm{~km} \mathrm{~s}^{-1}$ for $\mathrm{N}_{2} \mathrm{H}^{+}, 1.2,3.6,6,10,18,30$, and $48 \mathrm{Jy}$ beam $^{-1} \mathrm{~km} \mathrm{~s}^{-1}$ for $\mathrm{C}^{34} \mathrm{~S}$, and $3,6,12,24,48,96$, and 144 $\mathrm{Jy} \mathrm{beam}^{-1} \mathrm{~km} \mathrm{~s}^{-1}$ for $\mathrm{CH}_{3} \mathrm{OH}\left(2_{k}-1_{k}\right)$, and $3,9,15,25,45,60$, and 120 times $2.5 \mathrm{mJy} \mathrm{beam}^{-1}$ for the $3.16 \mathrm{~mm}$ continuum emission. The dashed circumference in the top panel represents the BIMA primary beam (50\% attenuation level). The synthesized beams are shown in the lower right-hand corner. The crosses pinpoint the positions of the four YSOs identified by Furuya et al. (2002). 


\subsection{Results}

In this section we analyze the line and continuum observations carried out with the BIMA and PdBI interferometers toward G24.78+0.08. Subsequently to the lower angular resolution BIMA data, we present the higher angular resolution PdBI data. By presenting the results in such a way, the analysis of G24.78+0.08 starts with the study of the large scale molecular clumps, and then continues with the study of the smaller scale embedded cores.

\subsection{1. $\mathrm{N}_{2} \mathrm{H}^{+}, \mathrm{C}^{34} \mathrm{~S}$ and $\mathrm{CH}_{3} \mathrm{OH}$}

$\mathrm{N}_{2} \mathrm{H}^{+}(1-0), \mathrm{C}^{34} \mathrm{~S}(2-1)$, and $\mathrm{CH}_{3} \mathrm{OH}\left(2_{-1}-1_{-1}\right)$ E, $\left(2_{0}-1_{0}\right)$ $\mathrm{A}^{+},\left(2_{0}-1_{0}\right) \mathrm{E}$ were observed toward the hot core $\mathrm{G} 24.78+0.08$ with the BIMA interferometer. Figure 10 shows the integrated emission around the systemic velocity of $111 \mathrm{~km} \mathrm{~s}^{-1}$, overlaid on the $3.16 \mathrm{~mm}$ continuum emission, observed also with BIMA. As may be seen in this figure, a well defined molecular core is found in association with G24.78+0.08, although the low angular resolution of the BIMA maps $\left(\theta \sim 14^{\prime \prime}\right)$ makes it impossible to disentangle the emission of each core identified in the region by Furuya et al. (2002). This molecular clump has also been detected in several rotational transitions of CO and its isotopomers, and in CS (3-2) (Cesaroni et al. 2003). In Fig. 10 one can see that each species peaks at different positions: $\mathrm{C}^{34} \mathrm{~S}$ toward core $\mathrm{A}$, and $\mathrm{CH}_{3} \mathrm{OH}$ and $\mathrm{N}_{2} \mathrm{H}^{+}$ between cores $\mathrm{C}$ and $\mathrm{A}$, with the $\mathrm{N}_{2} \mathrm{H}^{+}$emission peaking much closer to core $\mathrm{C}$. This suggests that each species could be tracing different physical conditions and/or chemical evolution of the molecular gas toward the YSOs. The lower resolution of our observations, however, did not allow us to investigate this in more detail. Taking into account that we have detected rotating structures associated with some of the individual cores embedded in G24.78+0.08 (see Paper I), we also searched for the presence of a global velocity gradient that could be indicative of large scale rotation of the whole cloud. To do this we fitted the $C^{34} \mathrm{~S}(2-1)$ lines with a Gaussian at each point in order to measure shifts from the systemic velocity of the cloud, and we also minimized the $\chi^{2}$ measure of the difference between a model with a linear velocity gradient and the $C^{34} S(2-1)$ data at each point. However, no significant rotation was detected by either of the two methods, except for the central part of the cloud where the velocity gradient is more evident and consistent with that found on a smaller scale in $\mathrm{CH}_{3} \mathrm{CN}$.

A second molecular clump has clearly been detected in $\mathrm{N}_{2} \mathrm{H}^{+}$and is also visible in $\mathrm{CH}_{3} \mathrm{OH}$ and marginally in $\mathrm{C}^{34} \mathrm{~S}$, toward the northwest of G24.78+0.08 (see Fig. 10). Millimeter continuum emission has also been detected in our $3.16 \mathrm{~mm}$ low angular resolution $\left(\theta \sim 18^{\prime \prime}\right)$ BIMA observations toward the northwest. This continuum emission peaks at the position of an extended HII region visible at $20 \mathrm{~cm}$ in the NVSS survey (Condon et al. 1998), and is probably associated with it. However, as can be seen in the figure, there is also some continuum emission associated with the $\mathrm{N}_{2} \mathrm{H}^{+}$clump. Submillimeter emission associated with this clump has also been mapped at 850 and $450 \mu \mathrm{m}$ with the Submillimeter Common-User Bolometric Array (SCUBA) by Walsh et al. (2003). In their

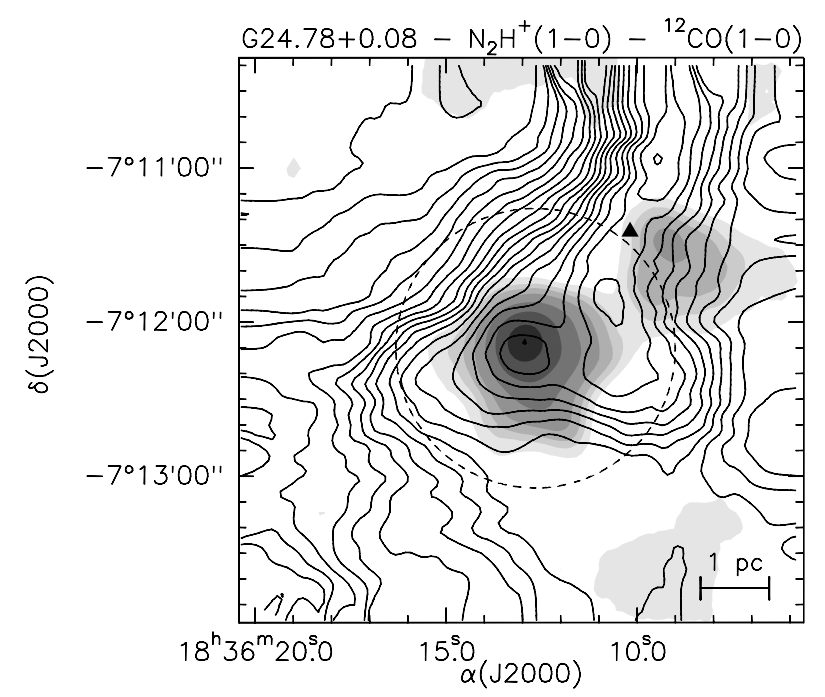

Fig. 11. Overlay of the BIMA $\mathrm{N}_{2} \mathrm{H}^{+}(1-0)$ integrated emission (greyscale), and the ${ }^{12} \mathrm{CO}(1-0)$ integrated emission obtained with the IRAM 30-m telescope by Cesaroni et al. (2003; contours) toward G24.78+0.08. The ${ }^{12} \mathrm{CO}$ emission has been integrated under the line, and the contour levels range from 4 to $194 \mathrm{~K} \mathrm{~km} \mathrm{~s}^{-1}$ in steps of $10 \mathrm{~K} \mathrm{~km} \mathrm{~s}^{-1}$. Contours for $\mathrm{N}_{2} \mathrm{H}^{+}$are the same as in Fig. 10. The triangle pinpoints the position of the peak of the $20 \mathrm{~cm}$ continuum emission extracted from the NVSS database. The dashed circumference represents the BIMA primary beam (50\% attenuation level).

maps lower-level extended emission is also visible connecting the two clumps in the region. The clump has also been detected in several rotational transitions of $\mathrm{CO}$ and its isotopomers (Cesaroni et al. 2003). However, the peak of the ${ }^{12} \mathrm{CO}$ emission is displaced from the position of the $\mathrm{N}_{2} \mathrm{H}^{+}$emission peak (see Fig. 11), unlike the molecular clump at the center of the map for which both $\mathrm{CO}$ and $\mathrm{N}_{2} \mathrm{H}^{+}$peak at the same position. The lack of $\mathrm{CO}$ emission toward this northern clump could be due to an opacity effect or to molecular depletion. For low-mass starless cores, the abundance of some molecules, including CO and CS, dramatically decreases toward the core center, with respect to the outer region. On the other hand, molecules such as $\mathrm{N}_{2} \mathrm{H}^{+}$are found to have constant abundances (e.g. Tafalla et al. 2002). These results are explained by the depletion of carbonand sulfur-bearing molecules onto dust grains at high densities (a few times $10^{4} \mathrm{~cm}^{-3}$ ) and low temperatures occurring in dense core interiors (e.g. Bergin \& Langer 1997). Nitrogen bearing molecules are instead unaffected by this process up to densities of several times $10^{5} \mathrm{~cm}^{-3}$, or even $10^{6} \mathrm{~cm}^{-3}$. In order to assess whether depletion is the cause of the lack of CO emission we need to estimate the opacity and the temperature of this northern clump. However, only the CO (1-0) and (2-1) lines have been observed toward this clump: therefore additional observations of an optically thin CO isotopomer and of a good temperature tracer are needed. Unfortunately, the energy of the three $\mathrm{CH}_{3} \mathrm{OH}\left(2_{k}-1_{k}\right)$ transitions observed with BIMA are too similar to derive any temperature by means of the RD method. If the $\mathrm{CO}$ freeze-out is confirmed, the northern clump would represent the first example of a chemically depleted high-mass clump. 


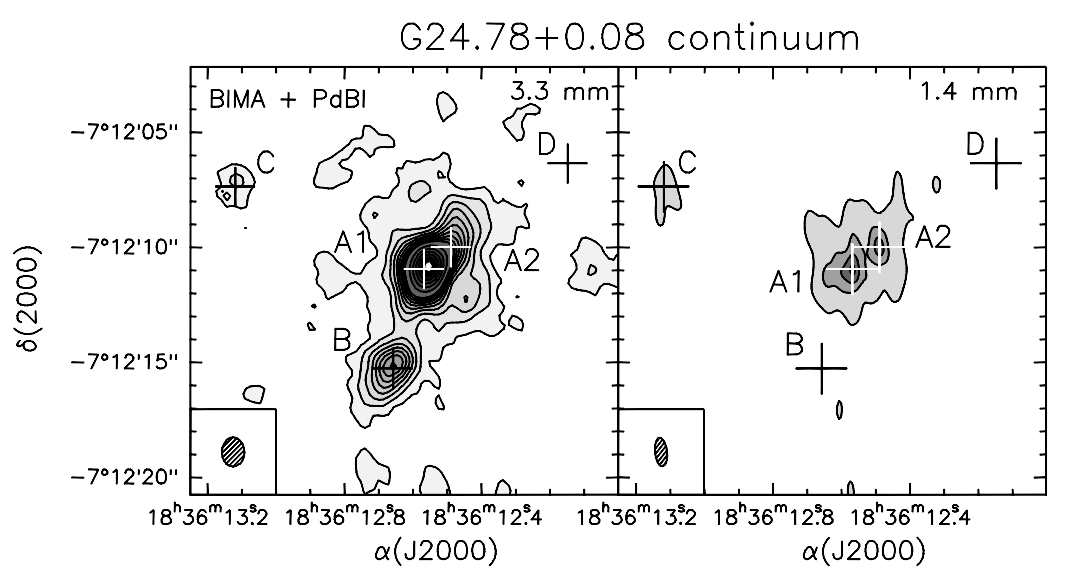

Fig. 12. Combined BIMA+PdBI map of the $3.3 \mathrm{~mm}$ continuum emission (left panel), and PdBI map of the 1.4 mm continuum emission (right panel) continuum emission toward the core of G24.78+0.08. The contour levels range from 2 to $20 \mathrm{mJy}_{\text {beam }}^{-1}$ in steps of $2 \mathrm{mJy}^{-1}$ beam ${ }^{-1}$ and from 20 to $80 \mathrm{mJy}_{\text {beam }}^{-1}$ in steps of $10 \mathrm{mJy}_{\text {beam }}^{-1}$ for the $3.3 \mathrm{~mm}$ map, and from 20 to $200 \mathrm{mJy} \mathrm{beam}^{-1}$ in steps of $60 \mathrm{mJy} \mathrm{beam}^{-1}$ for the $1.4 \mathrm{~mm}$ map. The synthesized beam is shown in the lower left-hand corner. The crosses mark the position of the YSOs identified in the region (Furuya et al. 2002; Paper I; see Sect. 4.1.2).

\subsubsection{Continuum emission}

In Fig. 12 we show the maps of the combined BIMA + PdBI $3.3 \mathrm{~mm}$ continuum emission and the PdBI $1.4 \mathrm{~mm}$ continuum emission toward G24.78+0.08. As mentioned in Sect. 4.1.1, $3.16 \mathrm{~mm}$ continuum low angular resolution $\left(\theta \sim 18^{\prime \prime}\right)$ BIMA maps are shown in Fig. 10. The highest angular resolution $\left(\theta \sim 1^{\prime \prime}\right)$ observations at $3.3 \mathrm{~mm}$ have resolved the continuum emission into three sources, A, B, and C, already detected at millimeter wavelengths by Furuya et al. (2002). The continuum emission at $3.3 \mathrm{~mm}$ is dominated by an extended structure surrounding cores $\mathrm{A}$ and $\mathrm{B}$. The strongest core detected at the $3.3 \mathrm{~mm}$ wavelength in the region is core A. The emission clearly shows two components, a centrally peaked source, plus an extended component elongated toward the northwest. The $1.4 \mathrm{~mm}$ dust emission associated with core $\mathrm{A}$ has been resolved into two separate cores, cores A1 and A2 (see Fig. 12; and Paper I), with core A2 located in the direction of the elongation toward NW seen at $3.3 \mathrm{~mm}$. The two cores are separated by $\sim 1$ '. 5 or $\sim 11500 \mathrm{AU}$. The positions, fluxes and deconvolved diameters of the cores are given in Table 3. The other core detected at both wavelengths is $\mathrm{C}$. The continuum emission associated with this YSO is much less extended, and it has only been resolved at $1.4 \mathrm{~mm}$. The measured flux densities of cores $\mathrm{A}$ and $\mathrm{C}$ at $3.3 \mathrm{~mm}$ are consistent with the previous measurements by Furuya et al. (2002; see their Fig. 3). At 1.4 mm, the sum of the flux densities of cores A1 and A2 is consistent with the expected flux density for core $\mathrm{A}$, while for $\mathrm{C}$ the flux that we have measured is slightly lower, but still consistent, with the model predictions of Cesaroni et al. (2003). The third source detected in the region at $3.3 \mathrm{~mm}$ is the UC HII region B (Codella et al. 1997; see Fig 12), which is slightly elongated in the southeast-northwest direction. This has not been detected at $1.4 \mathrm{~mm}$; however, the $1.4 \mathrm{~mm}$ flux expected on the basis of the results of Cesaroni et al. (2003) is lower than the $3 \sigma$ noise level of our map, which is about $20 \mathrm{mJy}_{\text {beam }}^{-1}$. The flux at $3.3 \mathrm{~mm}$ is also consistent with the values of Cesaroni et al. (2003). Finally, we have not detected at any wavelength core $\mathrm{D}$, which has been detected at 2.6 and $2 \mathrm{~mm}$ by

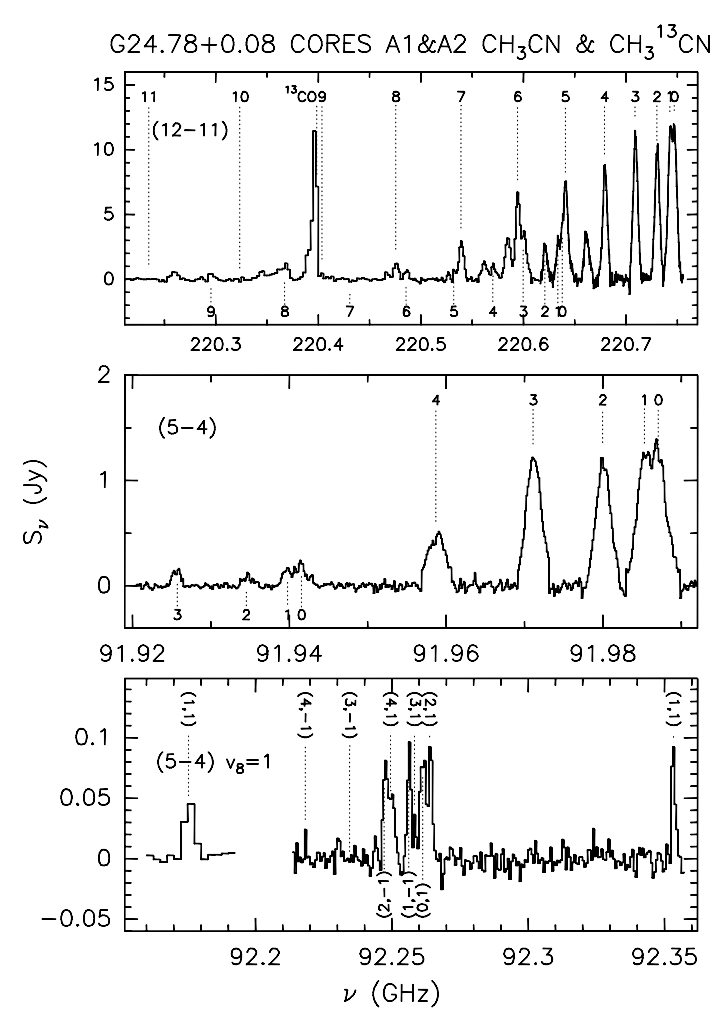

Fig. 13. Same as Fig. 2 for cores $\mathrm{A} 1+\mathrm{A} 2$ in $\mathrm{G} 24.78+0.08$.

Furuya et al. (2002). However, the expected flux of this core at 3.3 and $1.4 \mathrm{~mm}$, according to the predictions of Cesaroni et al. (2003), falls below the $3 \sigma$ noise level of our maps, 2 and $20 \mathrm{mJy}$, respectively.

\subsection{3. $\mathrm{CH}_{3} \mathrm{CN}$ and $\mathrm{CH}_{3}^{13} \mathrm{CN}$}

PdBI spectra of the $\mathrm{CH}_{3} \mathrm{CN}(12-11)$ and $(5-4), \mathrm{CH}_{3}{ }^{13} \mathrm{CN}$ (12-11) and (5-4), and $\mathrm{CH}_{3} \mathrm{CN}$ (5-4) $v_{8}=1$ lines toward cores A1 and A2 in G24.78+0.08 are shown in Fig. 13. 


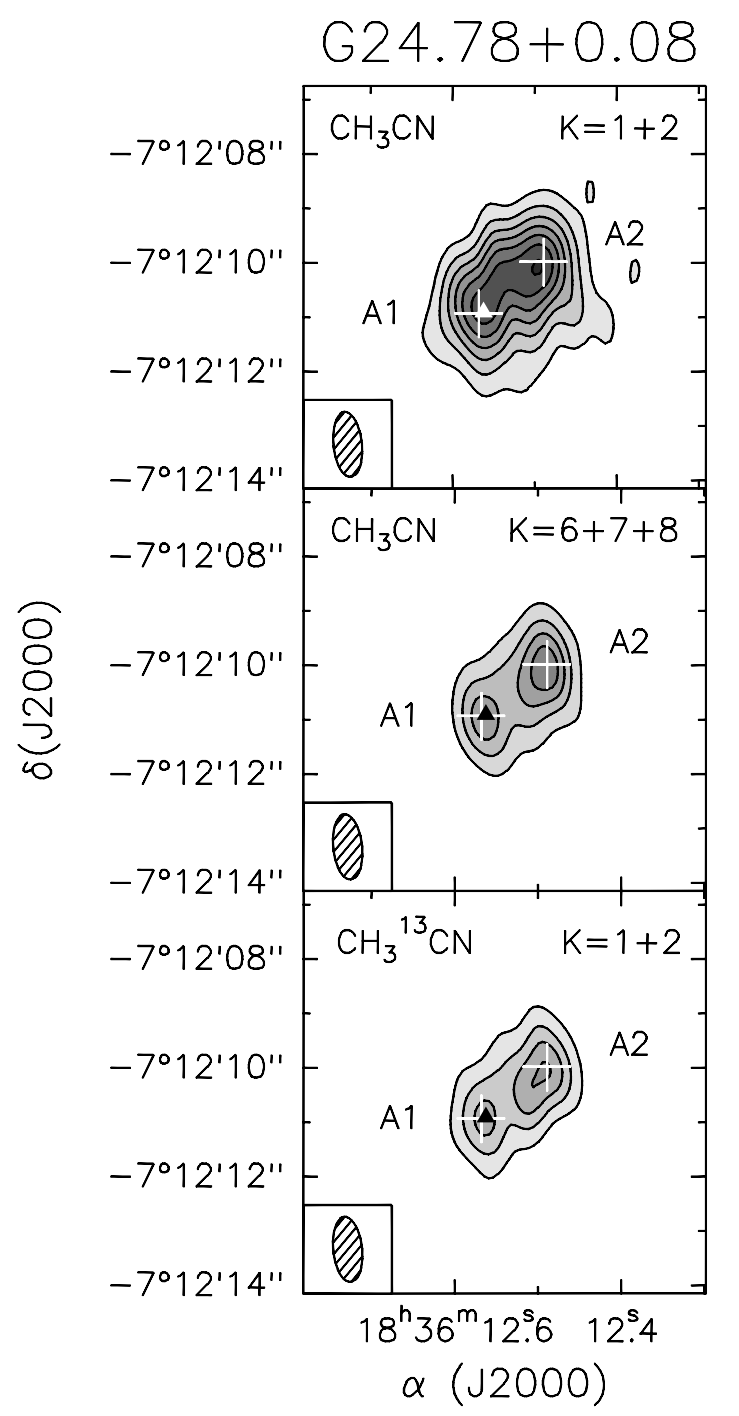

Fig. 14. $\mathrm{PdBI}$ intensity maps of the $\mathrm{CH}_{3} \mathrm{CN}(12-11)$ emission averaged under the $K=1$ and 2 components (top panel), under the $K=6$, 7 , and 8 components (middle panel), and of the $\mathrm{CH}_{3}{ }^{13} \mathrm{CN}(12-11)$ emission under the $K=1$ and 2 components (bottom panel) toward cores $\mathrm{A} 1$ and $\mathrm{A} 2$ in $\mathrm{G} 24.78+0.08$. The contour levels range from 0.14 to $0.98 \mathrm{Jy} \mathrm{beam}^{-1}$ (top panel), and to $0.56 \mathrm{Jy}^{\text {beam }}{ }^{-1}$ (middle and bottom panels), in steps of $0.14 \mathrm{Jy}^{\text {beam }^{-1}}$. The synthesized beam is shown in the lower left-hand corner. The white crosses denote the position of the $1.4 \mathrm{~mm}$ continuum emission peaks. The triangle marks the position of the VLA $1.3 \mathrm{~cm}$ continuum emission peak (Codella et al. 1997).

The spectra have been obtained averaging the emission over the $3 \sigma$ contour level area, which surrounds both cores A1 and A2. Figure 14 shows the PdBI maps of the $\mathrm{CH}_{3} \mathrm{CN}$ (12-11) emission averaged under the $K=1$ and 2 components and under the $K=6,7$ and 8 components, and the $\mathrm{CH}_{3}{ }^{13} \mathrm{CN}$ emission averaged under the $K=1$ and 2 components. Figure 15 shows the maps of the $\mathrm{CH}_{3} \mathrm{CN}$ (5-4) emission averaged under the $K=0$ to 4 components, the $\mathrm{CH}_{3}{ }^{13} \mathrm{CN}$ emission averaged under the $K=0$ to 3 components and the $\mathrm{CH}_{3} \mathrm{CN} v_{8}=1$ emission averaged under all observed transitions.

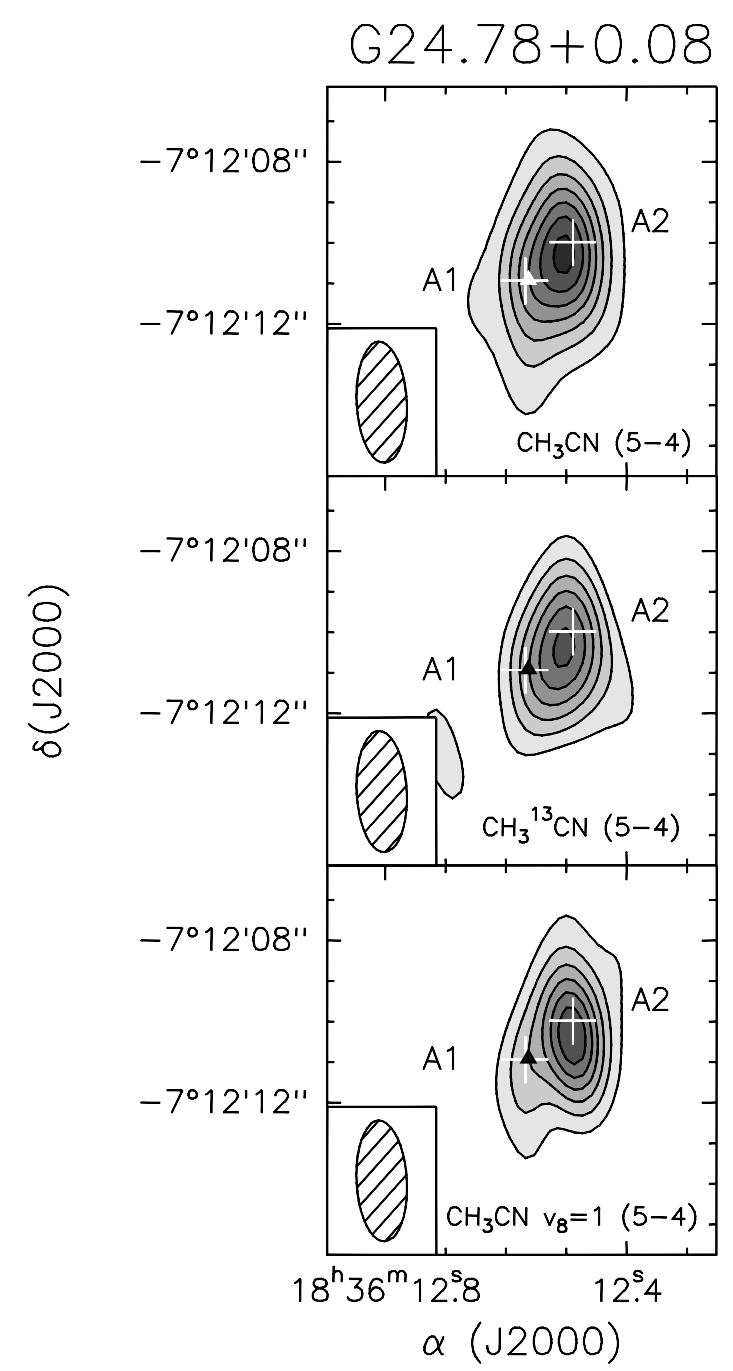

Fig. 15. $\mathrm{PdBI}$ intensity maps of the $\mathrm{CH}_{3} \mathrm{CN}$ (5-4) (top panel) emission averaged under the $K=0$ to 4 components, the $\mathrm{CH}_{3}{ }^{13} \mathrm{CN}$ (middle panel) emission averaged under the $K=0$ to 3 components, and of the $v_{8}=1$ emission (bottom panel) averaged under the $(1,1),(2,1),(0,1)$, $(3,1),(1,-1),(4,1),(2,-1),(3,-1),(4,-1)$, and $(1,1)$ transitions toward cores A1 and A2 in G24.78+0.08. The contour levels range from 0.05 to $0.35 \mathrm{Jy} \mathrm{beam}^{-1}$ in steps of $0.05 \mathrm{Jy}_{\text {beam }}^{-1}$ (top panel), from 0.01 to $0.06 \mathrm{Jy}$ beam $^{-1}$ in steps of $0.01 \mathrm{Jy} \mathrm{beam}^{-1}$ (middle panel), and from 0.005 to $0.030 \mathrm{Jy}^{\text {beam }}{ }^{-1}$ in steps of $0.005 \mathrm{Jy}^{\text {beam }}{ }^{-1}$ (bottom panel). The synthesized beam is shown in the lower left-hand corner. The white crosses denote the position of the $1.4 \mathrm{~mm}$ continuum emission peaks. The triangle marks the position of the VLA $1.3 \mathrm{~cm}$ continuum emission peak (Codella et al. 1997).

The methyl cyanide emission of the two cores has not been resolved at $3.3 \mathrm{~mm}$ because the separation between them is smaller than the synthesized beam, whereas they have been resolved at $1.4 \mathrm{~mm}$ (Figs. 14 and 15). For the sake of comparison with the $\mathrm{CH}_{3} \mathrm{CN}$ (5-4) lines, for the $\mathrm{CH}_{3} \mathrm{CN}$ (12-11) emission we also decided to plot the spectra integrated over a region surrounding both cores. As can be seen in Fig. 13, several $K$-components of the different rotational transitions of methyl cyanide were clearly detected. No methyl cyanide emission has been detected toward cores B or D with the PdBI. For 
Table 7. $\mathrm{CH}_{3} \mathrm{CN}$ and $\mathrm{CH}_{3}{ }^{13} \mathrm{CN}(12-11)$ line parameters for core $\mathrm{A} 1$ in $\mathrm{G} 24.78+0.08$.

\begin{tabular}{|c|c|c|c|c|c|}
\hline $\begin{array}{c}\text { Line } \\
K\end{array}$ & $\begin{array}{c}V_{\mathrm{LSR}} \\
\left(\mathrm{km} \mathrm{s}^{-1}\right)\end{array}$ & $\begin{array}{c}F W H M \\
\left(\mathrm{~km} \mathrm{~s}^{-1}\right)\end{array}$ & $\begin{array}{l}T_{\mathrm{B}}^{a} \\
(\mathrm{~K})\end{array}$ & $\begin{array}{c}\int_{\left(\mathrm{K} \mathrm{km} \mathrm{s}^{-1}\right)} T_{\mathrm{B}} \mathrm{d} V \\
\end{array}$ & $\begin{array}{c}\mathrm{rms} \\
\left(\mathrm{K} \mathrm{km} \mathrm{s}^{-1}\right)\end{array}$ \\
\hline & \multicolumn{5}{|c|}{$\mathrm{CH}_{3} \mathrm{CN}$} \\
\hline 0 & $110.82 \pm 0.02$ & $7.25 \pm 0.03$ & $26.8 \pm 1.5$ & $207 \pm 3$ & 0.9 \\
\hline 1 & $"$ & $"$ & $27.6 \pm 1.5$ & $213 \pm 3$ & $"$ \\
\hline 2 & $"$ & $"$ & $28.3 \pm 1.5$ & $219 \pm 3$ & $"$ \\
\hline 3 & $"$ & $"$ & $30.3 \pm 1.5$ & $234 \pm 2$ & $"$ \\
\hline 4 & $110.82 \pm 0.05$ & $7.12 \pm 0.07$ & $23.6 \pm 1.3$ & $179 \pm 3$ & 1.0 \\
\hline 5 & $"$ & $"$ & $18.2 \pm 1.3$ & $207 \pm 3$ & $"$ \\
\hline 6 & $110.64 \pm 0.12$ & $6.90 \pm 0.16$ & $17.0 \pm 0.8$ & $125 \pm 5$ & 0.8 \\
\hline 7 & $"$ & $"$ & $7.7 \pm 0.8$ & $56 \pm 3$ & 0.8 \\
\hline 8 & $"$ & $"$ & $3.4 \pm 0.8$ & $25 \pm 3$ & $"$ \\
\hline 9 & $"$ & $"$ & $b$ & $b$ & $"$ \\
\hline 10 & $"$ & $"$ & $<0.8$ & & $"$ \\
\hline \multirow[t]{2}{*}{11} & $"$ & $"$ & $<0.8$ & & $"$ \\
\hline & \multicolumn{5}{|c|}{$\mathrm{CH}_{3}{ }^{13} \mathrm{CN}$} \\
\hline 0 & $110.82 \pm 0.05$ & $7.12 \pm 0.07$ & $\bar{c}$ & $\bar{c}$ & 1.0 \\
\hline 1 & $"$ & $"$ & $7.0 \pm 1.3$ & $53 \pm 3$ & $"$ \\
\hline 2 & $"$ & $"$ & $6.6 \pm 1.3$ & $50 \pm 2$ & $"$ \\
\hline 3 & $110.64 \pm 0.12$ & $6.90 \pm 0.16$ & $8.7 \pm 0.8$ & $64 \pm 5$ & 0.8 \\
\hline 4 & $110.64 \pm 0.10$ & $6.92 \pm 0.14$ & $2.6 \pm 0.8$ & $19 \pm 3$ & 0.7 \\
\hline 5 & $"$ & $"$ & $0.9 \pm 0.8^{d}$ & $6 \pm 3^{d}$ & $"$ \\
\hline 6 & $110.64 \pm 0.12$ & $6.90 \pm 0.16$ & $1.8 \pm 0.8$ & $13 \pm 3$ & 0.8 \\
\hline 7 & $110.68 \pm 0.72$ & $7.0^{e}$ & $<0.8$ & & 0.8 \\
\hline 8 & " & $"$ & $3.4 \pm 0.5^{f}$ & $26 \pm 5^{f}$ & $"$ \\
\hline 9 & $"$ & $"$ & $1.3 \pm 0.5$ & $10 \pm 5$ & $"$ \\
\hline
\end{tabular}

\footnotetext{
${ }^{a}$ Brightness temperature integrated over a $3 \sigma$ emission level area around core $\mathrm{A} 1$.

${ }^{b}$ Blended with ${ }^{13} \mathrm{CO}(2-1)$.

${ }^{c}$ Blended with $\mathrm{CH}_{3} \mathrm{CN} K=5$.

${ }^{d}$ Blended with $\mathrm{CH}_{3} \mathrm{CN} \mathrm{K}=7$.

${ }^{e}$ This parameter was held fixed in the fit.

${ }^{f}$ Possibly blended with wing emission of ${ }^{13} \mathrm{CO}(2-1)$.
}

core $\mathrm{C}$, only the $\mathrm{CH}_{3} \mathrm{CN}$ (5-4) emission has been barely detected, with an averaged intensity peak of $\sim 30 \mathrm{mJy}^{\text {beam }}{ }^{-1}$. Cores $\mathrm{B}$ and $\mathrm{C}$ have been detected in the $\mathrm{CH}_{3} \mathrm{CN}(8-7)$ transition by Furuya et al. (2002), hence the fact that they are not detected in the (12-11) lines could be due to these cores being significantly colder than cores A1 and A2. As done for the spectra toward $\mathrm{G} 31.41+0.31$, we fitted the multiple $K$-components of the different $\mathrm{CH}_{3} \mathrm{CN}$ and $\mathrm{CH}_{3}{ }^{13} \mathrm{CN}$ rotational transitions (see Sect. 3.1.2 for details of the fitting). The $\mathrm{CH}_{3} \mathrm{CN}$ and $\mathrm{CH}_{3}{ }^{13} \mathrm{CN}$ (12-11) line parameters for cores $\mathrm{A} 1$ and $\mathrm{A} 2$, individually, are given in Tables 7 and 8 , respectively, while the $\mathrm{CH}_{3} \mathrm{CN}$ and $\mathrm{CH}_{3}{ }^{13} \mathrm{CN}$ (5-4), and $\mathrm{CH}_{3} \mathrm{CN}$ (5-4) $v_{8}=1$ line parameters for the emission surrounding both cores A1 and A2 (hereafter A1+A2) are given in Tables 9 and 10 . As can be seen in Tables 7 and 8 the systemic velocities and the line widths derived for the (12-11) transition are quite similar for both cores: $V_{\mathrm{LSR}} \simeq 110.6-110.9 \mathrm{~km} \mathrm{~s}^{-1}$, and $F W H M \simeq 7 \mathrm{~km} \mathrm{~s}^{-1}$. The systemic velocity is also consistent with that found for the ground state and $v_{8}=1(5-4)$ transitions in cores $\mathrm{A} 1+\mathrm{A} 2$, although those derived from the ground state lines are slightly higher $\left(111.2-111.6 \mathrm{~km} \mathrm{~s}^{-1}\right)$. Regarding the line widths, the values derived for the (5-4) transitions are quite different, with $\sim 7.8 \mathrm{~km} \mathrm{~s}^{-1}$ for $\mathrm{CH}_{3} \mathrm{CN}$ and $\sim 4.6 \mathrm{~km} \mathrm{~s}^{-1}$ for $\mathrm{CH}_{3}{ }^{13} \mathrm{CN}$. As done for $\mathrm{G} 31.41+0.31$ (see Sect. 3.1.2), one can derive the ratio between the observed and the intrinsic line widths, which for $\mathrm{G} 24.78+0.08$ is $1.7 \pm 0.04$. On the other hand, the ratio derived from the optical depths of the $\mathrm{CH}_{3} \mathrm{CN}$ and $\mathrm{CH}_{3}{ }^{13} \mathrm{CN}$ (5-4) lines (assuming a relative abundance $\left[\mathrm{CH}_{3} \mathrm{CN}\right] /\left[\mathrm{CH}_{3}{ }^{13} \mathrm{CN}\right]=40$; see Wilson \& Rood 1994) ranges from 1.5 to 1.9 . Thus, also in this case, optical depth effects could be responsible for the variation of the line widths. The line widths found for (5-4) $v_{8}=1$ are slightly smaller but still consistent with those found for the (12-11) transition.

Both core A1 and A2 are clearly visible in Fig. 14, the emission associated with core A2 being the strongest for all the transitions. The emission of both cores has not been resolved at $3 \mathrm{~mm}$ (Fig. 15). As can be seen in Fig. 14, the morphology of the emission is quite similar for the different $K$-components of $\mathrm{CH}_{3} \mathrm{CN}$ and $\mathrm{CH}_{3}{ }^{13} \mathrm{CN}$ : one sees two compact cores, roughly coincident with the two $1.4 \mathrm{~mm}$ continuum cores A1 and A2, plus an extended component. Such a good agreement between the gas and the dust emission indicates that they are tracing 
Table 8. $\mathrm{CH}_{3} \mathrm{CN}$ and $\mathrm{CH}_{3}{ }^{13} \mathrm{CN}(12-11)$ line parameters for core $\mathrm{A} 2$ in $\mathrm{G} 24.78+0.08$.

\begin{tabular}{|c|c|c|c|c|c|}
\hline $\begin{array}{c}\text { Line } \\
K\end{array}$ & $\begin{array}{c}V_{\mathrm{LSR}} \\
\left(\mathrm{km} \mathrm{s}^{-1}\right)\end{array}$ & $\begin{array}{c}F W H M \\
\left(\mathrm{~km} \mathrm{~s}^{-1}\right)\end{array}$ & $\begin{array}{l}T_{\mathrm{B}}^{a} \\
(\mathrm{~K})\end{array}$ & $\begin{array}{c}\int_{\left(\mathrm{K} \mathrm{km} \mathrm{s}^{-1}\right)} T_{\mathrm{B}} \mathrm{d} V \\
\mathrm{~km}^{-1}\end{array}$ & $\begin{array}{c}\mathrm{rms} \\
\left(\mathrm{K} \mathrm{km} \mathrm{s}^{-1}\right)\end{array}$ \\
\hline & \multicolumn{5}{|c|}{$\mathrm{CH}_{3} \mathrm{CN}$} \\
\hline 0 & $110.68 \pm 0.03$ & $7.25 \pm 0.03$ & $22.0 \pm 1.6$ & $170 \pm 3$ & 1.1 \\
\hline 1 & $"$ & $"$ & $22.6 \pm 1.6$ & $174 \pm 3$ & $"$ \\
\hline 2 & " & " & $23.8 \pm 1.6$ & $184 \pm 3$ & $"$ \\
\hline 3 & $"$ & " & $26.2 \pm 1.6$ & $202 \pm 2$ & $"$ \\
\hline 4 & $110.59 \pm 0.07$ & $7.18 \pm 0.10$ & $20.8 \pm 0.9$ & $159 \pm 3$ & 1.3 \\
\hline 5 & $"$ & $"$ & $16.0 \pm 0.9$ & $123 \pm 5$ & $"$ \\
\hline 6 & $110.87 \pm 0.08$ & $6.74 \pm 0.11$ & $16.3 \pm 1.1$ & $117 \pm 3$ & 0.6 \\
\hline 7 & " & $"$ & $7.9 \pm 1.1$ & $57 \pm 2$ & $"$ \\
\hline 8 & $110.88 \pm 0.10$ & $6.66 \pm 0.13$ & $4.3 \pm 1.0$ & $30 \pm 3$ & 0.7 \\
\hline 9 & $110.50 \pm 0.60$ & $7.0^{b}$ & $c$ & $c$ & 0.8 \\
\hline 10 & $"$ & $"$ & $<0.8$ & & $"$ \\
\hline \multirow[t]{2}{*}{11} & $"$ & $"$ & $<0.8$ & & " \\
\hline & \multicolumn{5}{|c|}{$\mathrm{CH}_{3}{ }^{13} \mathrm{CN}$} \\
\hline 0 & $110.59 \pm 0.07$ & $7.18 \pm 0.10$ & $d$ & $d$ & 1.3 \\
\hline 1 & $"$ & $"$ & $6.1 \pm 0.9$ & $47 \pm 3$ & $"$ \\
\hline 2 & $"$ & $"$ & $6.0 \pm 0.9$ & $46 \pm 3$ & $"$ \\
\hline 3 & $110.87 \pm 0.08$ & $6.74 \pm 0.11$ & $9.3 \pm 1.1$ & $67 \pm 3$ & 0.6 \\
\hline 4 & $"$ & $"$ & $3.3 \pm 1.1$ & $24 \pm 2$ & $"$ \\
\hline 5 & $"$ & $"$ & $1.8 \pm 1.1^{e}$ & $13 \pm 3^{e}$ & " \\
\hline 6 & $110.88 \pm 0.10$ & $6.66 \pm 0.13$ & $2.1 \pm 1.0$ & $15 \pm 3$ & 0.7 \\
\hline 7 & $110.50 \pm 0.60$ & $7.0^{b}$ & $<0.8$ & & 0.8 \\
\hline 8 & $"$ & $"$ & $3.7 \pm 0.6^{f}$ & $27 \pm 5^{f}$ & $"$ \\
\hline 9 & $"$ & $"$ & $1.2 \pm 0.6$ & $9 \pm 5$ & $"$ \\
\hline
\end{tabular}

${ }^{a}$ Brightness temperature integrated over a $3 \sigma$ emission level area around core A2.

${ }^{b}$ This parameter was held fixed in the fit.

${ }^{c}$ Blended with ${ }^{13} \mathrm{CO}(2-1)$.

${ }^{d}$ Blended with $\mathrm{CH}_{3} \mathrm{CN} K=5$.

${ }^{e}$ Blended with $\mathrm{CH}_{3} \mathrm{CN} K=7$.

${ }^{f}$ Dominated by wing emission of ${ }^{13} \mathrm{CO}(2-1)$.

the same material. In addition, there is no clear evidence of any emission dip for lower $K$-components of $\mathrm{CH}_{3} \mathrm{CN}$ toward the position of the embedded sources in the two cores, as for $\mathrm{G} 31.41+0.31$. This suggests that the temperature gradient in cores A1 and A2 in G24.78+0.08 is less prominent than in $\mathrm{G} 31.41+0.31$. This is confirmed by the $T_{\text {rot }}$ measured in each core, as illustrated in Sect. 4.2.1. In Fig. 14 we also mark the position of the peak of the $1.3 \mathrm{~cm}$ continuum emission of the UC HII region detected with the VLA by Codella et al. (1997). Note that this HII region is associated with core A1, being located at $\sim 0$ '. 1 ( $\sim 800 \mathrm{AU}$ at the distance of the region) of the millimeter continuum source, whereas no free-free emission is reported toward core A2.

Figure 16 shows the BIMA integrated $\mathrm{CH}_{3} \mathrm{CN}(5-4)$ emission under the $K=0,1,2$, and 3 components toward cores A1 and A2. The cores have been clearly resolved in these observations $\left(\theta \sim 00^{\prime} 7\right)$. As can be seen in the figure the strongest emission comes from core A2, with the peak slightly displaced from that of the $1.4 \mathrm{~mm}$ continuum emission. On the other hand, the emission from core A1 is barely detected. This could be due to a temperature effect, core A1 being probably

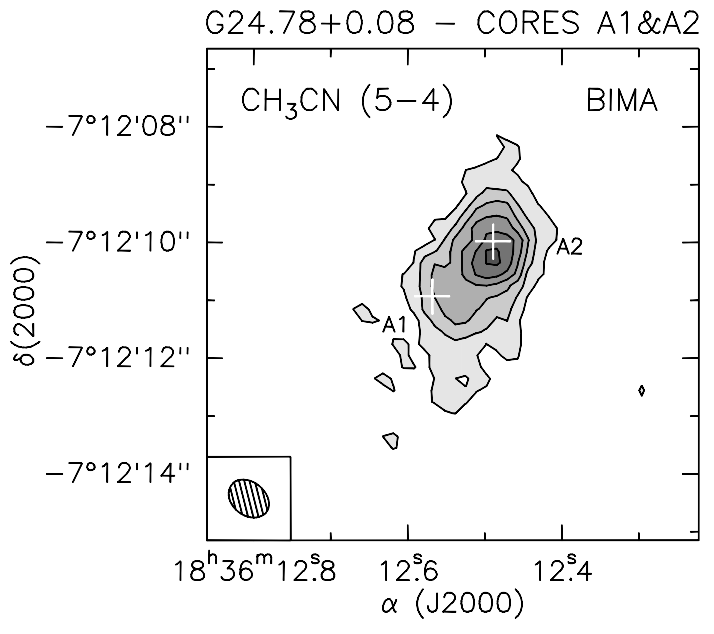

Fig. 16. BIMA integrated intensity map of the $\mathrm{CH}_{3} \mathrm{CN}$ (5-4) emission under the $K=0,1,2$, and 3 components toward cores A1 and A2 in G24.78+0.08. The contour levels are 3, 6, 9, 12, 15, and 18 times $0.35 \mathrm{Jy}_{\text {beam }}{ }^{-1} \mathrm{~km} \mathrm{~s}^{-1}$. The synthesized beam is shown in the lower left-hand corner. The white crosses mark the position of the $1.4 \mathrm{~mm}$ continuum emission peaks. 
Table 9. $\mathrm{CH}_{3} \mathrm{CN}$ and $\mathrm{CH}_{3}{ }^{13} \mathrm{CN}(5-4)$ line parameters for core $\mathrm{A} 1+\mathrm{A} 2$ in $\mathrm{G} 24.78+0.08^{a}$.

\begin{tabular}{|c|c|c|c|c|c|}
\hline $\begin{array}{c}\text { Line } \\
K\end{array}$ & $\begin{array}{c}V_{\mathrm{LSR}} \\
\left(\mathrm{km} \mathrm{s}^{-1}\right)\end{array}$ & $\begin{array}{c}F W H M \\
\left(\mathrm{~km} \mathrm{~s}^{-1}\right)\end{array}$ & $\begin{array}{l}T_{\mathrm{B}}^{b} \\
(\mathrm{~K})\end{array}$ & $\begin{array}{c}\int_{\left(\mathrm{K} \mathrm{km} \mathrm{s}^{-1}\right)} T_{\mathrm{B}} \mathrm{d} V \\
\mathrm{~km}^{-1}\end{array}$ & $\begin{array}{c}\mathrm{rms} \\
\left(\mathrm{K} \mathrm{km} \mathrm{s}^{-1}\right)\end{array}$ \\
\hline & \multicolumn{5}{|c|}{$\mathrm{CH}_{3} \mathrm{CN}$} \\
\hline 0 & $111.61 \pm 0.02$ & $7.74 \pm 0.03$ & $10.6 \pm 1.0$ & $87 \pm 1$ & 0.3 \\
\hline 1 & " & $"$ & $8.9 \pm 1.0$ & $70 \pm 1$ & $"$ \\
\hline 2 & " & " & $10.8 \pm 1.0$ & $89 \pm 1$ & $"$ \\
\hline 3 & $"$ & $"$ & $11.6 \pm 1.0$ & $96 \pm 1$ & $"$ \\
\hline \multirow[t]{2}{*}{4} & $"$ & $"$ & $4.9 \pm 1.0$ & $40 \pm 1$ & $"$ \\
\hline & \multicolumn{5}{|c|}{$\mathrm{CH}_{3}{ }^{13} \mathrm{CN}$} \\
\hline 0 & $111.20 \pm 0.08$ & $4.58 \pm 0.09$ & $2.00 \pm 0.27$ & $9.8 \pm 0.4$ & 0.18 \\
\hline 1 & $"$ & $"$ & $1.51 \pm 0.27$ & $7.39 \pm 0.35$ & $"$ \\
\hline 2 & $"$ & " & $0.86 \pm 0.27$ & $4.21 \pm 0.35$ & $"$ \\
\hline 3 & " & $"$ & $1.33 \pm 0.27$ & $6.50 \pm 0.35$ & " \\
\hline
\end{tabular}

${ }^{a}$ Emission integrated over a region surrounding cores A1 and A2 in G24.78+0.08.

${ }^{b}$ Brightness temperature integrated over a $3 \sigma$ emission level area.

Table 10. $\mathrm{CH}_{3} \mathrm{CN}(5-4) v_{8}=1$ line parameters for core $\mathrm{A} 1+\mathrm{A} 2$ in $\mathrm{G} 24.78+0.08^{a}$.

\begin{tabular}{lccccc}
\hline \hline Line & $\begin{array}{c}V_{\mathrm{LSR}} \\
\left(\mathrm{km} \mathrm{s}^{-1}\right)\end{array}$ & $\begin{array}{c}F W H M \\
\left(\mathrm{~km} \mathrm{~s}^{-1}\right)\end{array}$ & $\begin{array}{c}T_{\mathrm{B}}^{b} \\
(\mathrm{~K})\end{array}$ & $\begin{array}{c}\int T_{\mathrm{B}} \mathrm{d} V \\
\left(\mathrm{~K} \mathrm{~km} \mathrm{~s}^{-1}\right)\end{array}$ & $\begin{array}{c}\mathrm{rms} \\
\left(\mathrm{K} \mathrm{km} \mathrm{s}^{-1}\right)\end{array}$ \\
\hline$(1,1)$ & $110.61 \pm 0.18$ & $6.26 \pm 0.19$ & $0.77 \pm 0.14$ & $5.15 \pm 0.34$ & 0.07 \\
$(2,1)$ & $110.84 \pm 0.12$ & $5.78 \pm 0.13$ & $0.82 \pm 0.14$ & $5.04 \pm 0.32$ & 0.07 \\
$(0,1)$ & $"$ & $"$ & $0.91 \pm 0.14$ & $5.61 \pm 0.32$ & $"$ \\
$(3,1)$ & $"$ & $"$ & $0.22 \pm 0.14$ & $1.38 \pm 0.31$ & $"$ \\
$(1,-1)$ & $"$ & $"$ & $0.83 \pm 0.14$ & $5.11 \pm 0.32$ & $"$ \\
$(4,1)$ & $110.61 \pm 0.18$ & $6.26 \pm 0.19$ & $0.60 \pm 0.14$ & $3.98 \pm 0.34$ & 0.07 \\
$(2,-1)$ & $"$ & $"$ & $0.73 \pm 0.14$ & $4.84 \pm 0.34$ & $"$ \\
$(3,-1)$ & $"$ & $"$ & $<0.07$ & & $"$ \\
$(4,-1)$ & $110.61 \pm 0.18$ & $6.26 \pm 0.19$ & $0.11 \pm 0.14$ & $0.71 \pm 0.32$ & 0.07 \\
$(1,1)$ & $110.61^{c}$ & $6.26^{c}$ & $0.89 \pm 0.09^{d}$ & $5.94 \pm 0.52^{e}$ & 0.04 \\
\hline
\end{tabular}

${ }^{a}$ Emission integrated over a region surrounding G24.78+0.08 cores A1 and A2.

${ }^{b}$ Brightness temperature integrated over a $3 \sigma$ emission level area.

${ }^{c}$ This parameter was held fixed in the fit.

${ }^{d}$ Computed from the integrated line intensity.

${ }^{e}$ Measured with the command print area of CLASS.

hotter than core A2. We checked whether it could be an opacity effect by computing the optical depth in the cores from the ratio of $\mathrm{CH}_{3}{ }^{13} \mathrm{CN}$ and $\mathrm{CH}_{3} \mathrm{CN}(12-11) \mathrm{K}=2$ component (see Sect. 3.1.2). However, the optical depths obtained are similar for both cores, $\tau_{12} \sim 12$, suggesting that opacity cannot account for the difference in $\mathrm{CH}_{3} \mathrm{CN}$ (5-4) emission between both cores. In Fig. 17 we compare the $\mathrm{CH}_{3} \mathrm{CN}$ (5-4) maps obtained with BIMA and the PdBI. To compare them we have first reduced the resolution of the BIMA map by restoring the "clean" BIMA map with the same beam as the PdBI observations. As can be seen in this figure there is an excellent agreement between the two maps, which confirms that most of the $\mathrm{CH}_{3} \mathrm{CN}$ (5-4) emission is associated with core A2. Note that some extended emission is not shown by the BIMA map, probably due to the fact that it is filtered out by the interferometer due to the lack of short spacings. In Fig. 18 the same comparison is shown for the spectra obtained integrating the emission over the same region. Again, taking into account that some of the emission is probably filtered out by the BIMA interferometer, one can say that the agreement between both spectra is remarkable.

\subsection{Discussion}

\subsubsection{Temperature and column density}

We derived the rotational temperature and total column density of the methyl cyanide molecules by means of the RD method (see Sect. 3.1.2) for cores A1+A2 in G24.78+0.08 (see Fig. 19). The insufficient angular resolution of the $3 \mathrm{~mm}$ observations makes one unable to separate the emission from the two cores, and thus the emission has been integrated over an area of $\sim 4^{\prime \prime}$ of diameter $\left(\sim 12 \operatorname{arcsec}^{2}\right)$ that covers both core A1 and A2. As stated before, such a region would correspond to 


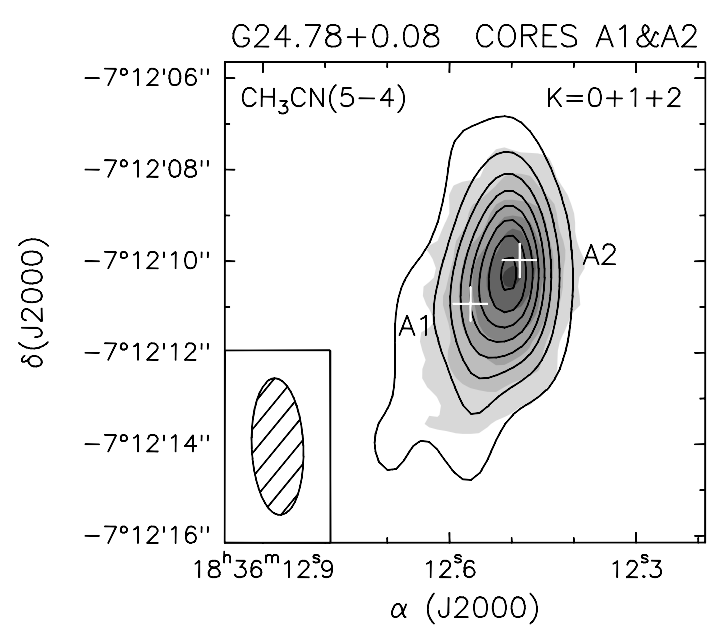

Fig. 17. Overlay of the integrated intensity map of the $\mathrm{CH}_{3} \mathrm{CN}(5-4)$ emission under the $K=0,1$, and 2 components obtained with the PdBI (contours) on the map obtained with BIMA (greyscale). The contour levels range from 0.05 to $0.35 \mathrm{Jy} \mathrm{beam}^{-1} \mathrm{~km} \mathrm{~s}^{-1}$ (greyscale) and to $0.40 \mathrm{Jy}^{\text {beam }}{ }^{-1} \mathrm{~km} \mathrm{~s}^{-1}$ (contours) in steps of $0.05 \mathrm{Jy} \mathrm{beam}^{-1} \mathrm{~km} \mathrm{~s}^{-1}$. The BIMA map has been obtained by restoring the "clean" map with the same beam than that of the PdBI observations (see text). The synthesized beam is shown in the lower left-hand corner.The white crosses mark the position of the $1.4 \mathrm{~mm}$ continuum emission peaks.

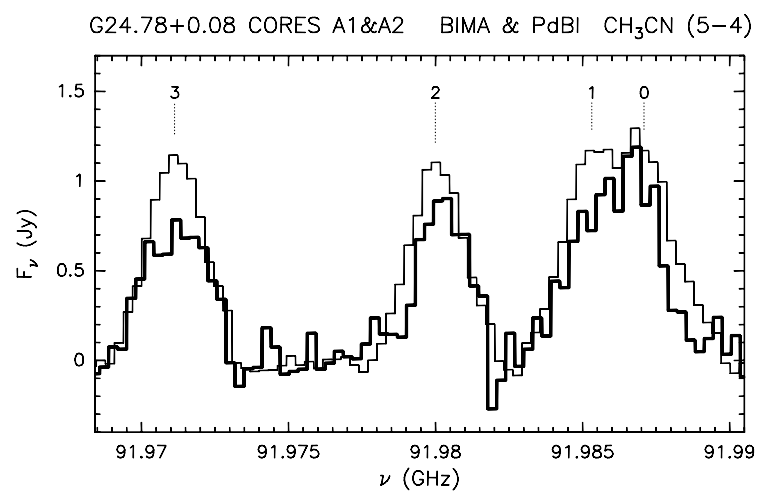

Fig. 18. Comparison between the BIMA (thick line) and the PdBI (thin line) $\mathrm{CH}_{3} \mathrm{CN}$ (5-4) spectra toward cores $\mathrm{A} 1+\mathrm{A} 2$ in $\mathrm{G} 24.78+0.08$. Both spectra have been obtained averaging the emission over the same region. The BIMA channel maps have been obtained by restoring the "clean" map with the same beam than that of the PdBI observations (see text). The number of the different $K$-components are marked with dashed lines in the upper part.

core A of Codella et al. (1997) and Furuya et al. (2002). As already done for $\mathrm{G} 31.41+0.31$, we used only the ground state transitions of the isotopomer $\mathrm{CH}_{3}{ }^{13} \mathrm{CN}$, and those from the $\mathrm{CH}_{3} \mathrm{CN} v_{8}=1$ state in the fit performed to the Boltzmann plot, because the ground level transitions (see Fig. 13) appear to be optically thick. We assumed a relative abundance $\left[\mathrm{CH}_{3} \mathrm{CN}\right] /\left[\mathrm{CH}_{3}{ }^{13} \mathrm{CN}\right]=40$ (see Wilson \& Rood 1994). The $T_{\text {rot }}$ and source averaged $N_{\mathrm{CH}_{3} \mathrm{CN}}$ that we derived by taking into account all transitions are $132 \mathrm{~K}$ and $2 \times 10^{16} \mathrm{~cm}^{-2}$, respectively. The $T_{\text {rot }}$ and $N_{\mathrm{CH}_{3} \mathrm{CN}}$ that we estimated from each set of $J+1 \rightarrow J$ transitions of $\mathrm{CH}_{3}{ }^{13} \mathrm{CN}$ are $102 \pm 5$ and $130 \pm 2 \mathrm{~K}$, and $1 \times 10^{16}$ and $2 \times 10^{16} \mathrm{~cm}^{-2}$ for the (5-4) and

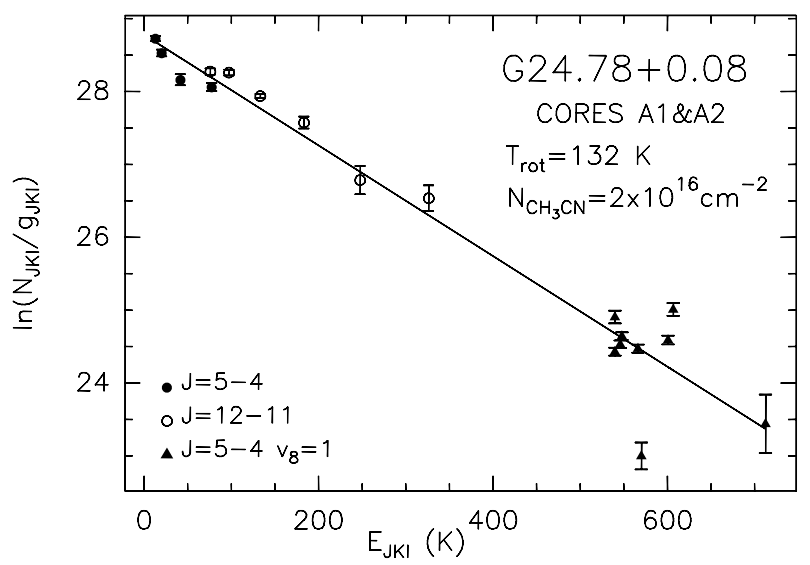

Fig. 19. Same as Fig. 6 for the cores A1+A2 in G24.78+0.08.

(12-11) transitions, respectively. Note that unlike the case of $\mathrm{G} 31.41+0.31$, for $\mathrm{G} 24.78+0.08$ these two estimates are quite similar, which could be indicating that all the transitions are tracing more or less the same gas or, as mentioned before, that the temperature gradient is less evident. From $\mathrm{NH}_{3}$ emission averaged over a similar area Codella et al. (1997) have derived a slightly lower kinetic temperature, $T_{\mathrm{k}}=87 \mathrm{~K}$ : this is not surprising because $\mathrm{NH}_{3}$ is probably tracing a different region, somewhat more extended and colder. The estimate of the gas column density derived by these authors is consistent with ours. Taking into account that with the angular resolution achieved at $1 \mathrm{~mm}$ it is possible to separate the emission from cores A1 and A2, we made rotation diagrams for the $\mathrm{CH}_{3}{ }^{13} \mathrm{CN}$ (12-11) for each of them separately (see Fig. 20). As seen in this plot, the $T_{\text {rot }}$ and source averaged $N_{\mathrm{CH}_{3} \mathrm{CN}}$ are similar for both cores.

In Fig. 21 we show the $T_{\text {rot }}$ map and the $N_{\mathrm{CH}_{3} \mathrm{CN}}$ map overlaid on the $\mathrm{CH}_{3}{ }^{13} \mathrm{CN}$ (12-11) emission averaged under the $K=1$ and 2 components toward cores A1 and A2. We computed the maps as done for $\mathrm{G} 31.41+0.31$ (see Sect. 3.1.2). This figure shows that both cores have similar $T_{\text {rot }}, \sim 120-150 \mathrm{~K}$, consistent with the source averaged temperatures derived in the previous paragraph. In Sect. 4.1.3 we proposed that core A1 could be hotter than A2, because the former is barely detected in $\mathrm{CH}_{3} \mathrm{CN}$ (5-4) (see Figs. 16 and 17). The fact that we derive similar temperatures for both cores by means of the RD method rules out this hypothesis and indicates that the assumption of LTE is not valid in this case. In order to explain the difference in $\mathrm{CH}_{3} \mathrm{CN}$ (5-4) emission, one should take into account the IR radiation field of the UC HII region associated with this core (see Fig. 14). Figure 21 also shows that there is no clear radial temperature variation in the cores, unlike for $\mathrm{G} 31.41+0.31$ (see Fig. 7). Regarding the column density map, $N_{\mathrm{CH}_{3} \mathrm{CN}}$, one can see that there are two maxima roughly at the position of the methyl cyanide and millimeter continuum emission peaks for cores A1 and A2 (Fig.21), confirming that for these two cores, $\mathrm{CH}_{3} \mathrm{CN}$ and dust trace the same material.

\subsubsection{Nature of core $A$ and evolutionary sequence}

Core A was first detected at $1.3 \mathrm{~cm}$ by Codella et al. (1997) as a very compact and dense unresolved $\mathrm{NH}_{3}$ core. The gas and 


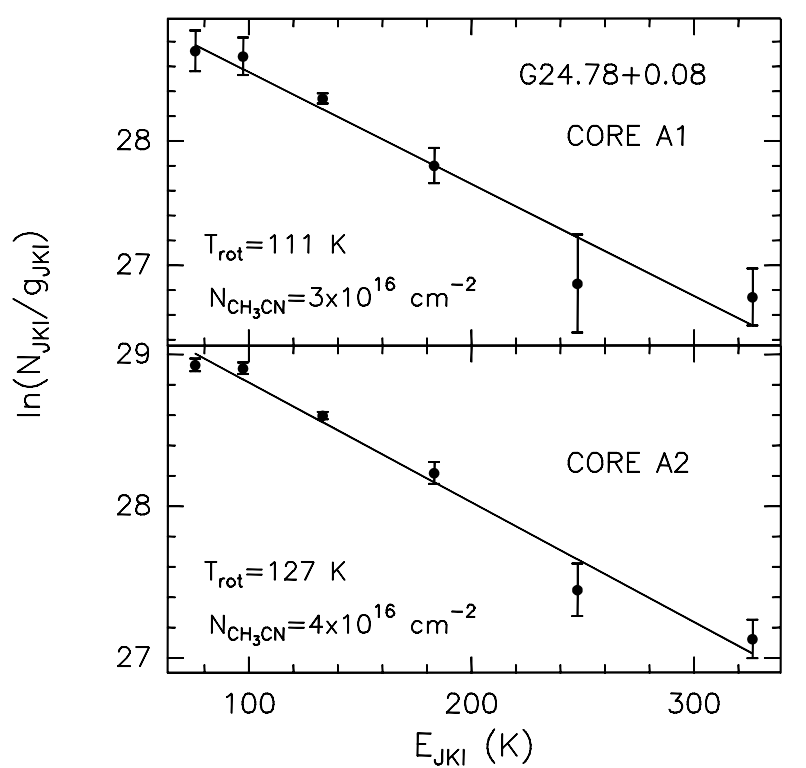

Fig. 20. Rotation diagram of $\mathrm{CH}_{3}{ }^{13} \mathrm{CN}(12-11)$ for cores $\mathrm{A} 1$ (top) and A2 (bottom) in G24.78+0.08, with superimposed fits.

dust millimeter emission from this core is the strongest and most extended of all the cores detected in the region. In addition, Furuya et al. (2002) have mapped a bipolar molecular outflow that is probably powered by a YSO embedded in the core. As already mentioned, thanks to the higher angular resolution of our PdBI millimeter observations, we have resolved core A into two separate cores, A1 and A2 (Paper I). These have been detected in gas and dust emission and both could be hosting high-mass YSOs. In such a case, the YSOs might be in different evolutionary stages, because the two cores hosting them have roughly the same mass $\left(\sim 130 M_{\odot}\right.$ for A1 and $\sim 80 M_{\odot}$ for A2; see Paper I), but only A1 has been found in association with an UC HII region, i.e. a more evolved YSO (see Fig. 14). The UC HII region has a diameter (at $50 \%$ of the peak emission) of $\sim 0$ ' 15 or $\sim 1200$ AU and is hence very small and probably in an early stage of its evolution. Regarding the bipolar outflow detected by Furuya et al. (2002) in association with $\mathrm{A}$, it is not evident which of the cores, $\mathrm{A} 1$ or $\mathrm{A} 2$, hosts the powering source. Core A1 lies slightly closer to the geometrical center of the outflow, but the small separation between the cores and the fact that they are aligned along the outflow axis make it difficult to establish any association unambiguously. Sensitive mid-IR observations would be very helpful to assess the YSO content of each core and possibly constrain their evolutionary state.

Furuya et al. (2002) have concluded that the hot core G24.78+0.08 contains at least 4 high-mass YSOs with different ages: more precisely, $t_{\mathrm{B}}>t_{\mathrm{A}}>t_{\mathrm{C}}>t_{\mathrm{D}}$. In the light of our results, we can now infer that the embedded YSOs could be 5, A1 probably being older than $\mathrm{A} 2$. In addition, the fact that source $\mathrm{C}$ is colder $(T \simeq 30 \mathrm{~K}$; see Codella et al. 1997) than source A2 ( $T \simeq 127 \mathrm{~K}$; see Sect. 4.2.1) suggests that $\mathrm{A} 2$ is probably older than C. Thus, the ages of the YSOs in G24.78+0.08 seem to be in the order $t_{\mathrm{B}}>t_{\mathrm{A} 1}>t_{\mathrm{A} 2}>t_{\mathrm{C}}>t_{\mathrm{D}}$.

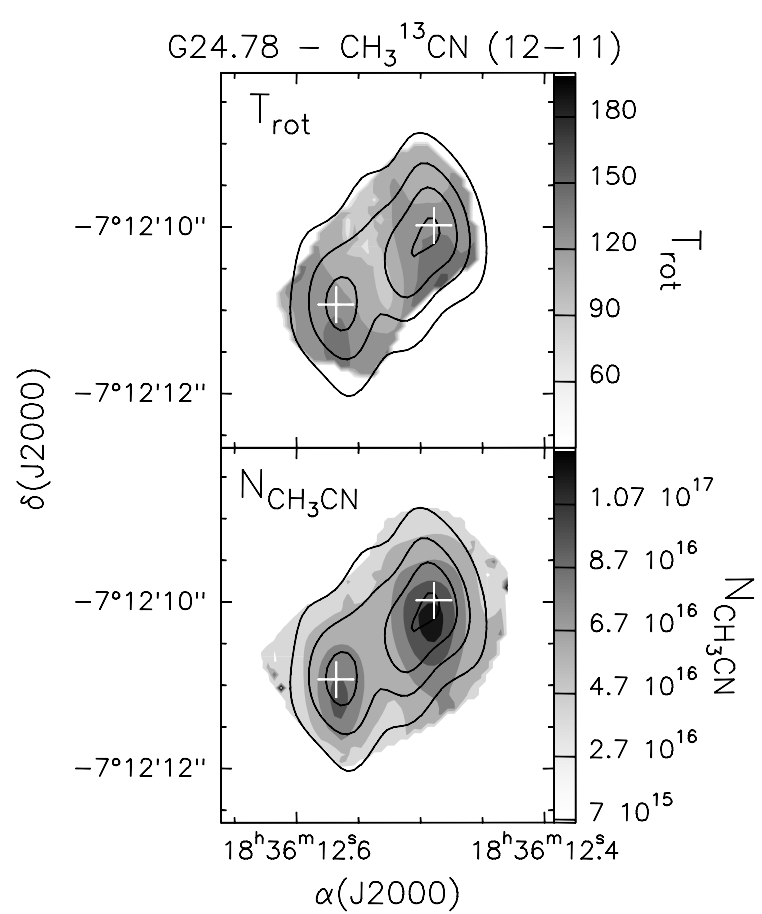

Fig. 21. Overlay of the $\mathrm{PdBI} \mathrm{CH}{ }_{3}{ }^{13} \mathrm{CN}(12-11)$ emission averaged under the $K=1$ and 2 components (contours) on the $T_{\text {rot }}$ map (top panel) and the $N_{\mathrm{CH}_{3} \mathrm{CN}}$ map (bottom panel) in greyscale, derived by fitting the Boltzmann plots at each position, toward cores A1 and A2 in $\mathrm{G} 24.78+0.08$. The contour levels range from 0.14 to $0.56 \mathrm{Jy} \mathrm{beam}^{-1}$ in steps of $0.56 \mathrm{Jy} \mathrm{beam}^{-1}$. Greyscale levels range from 60 to $180 \mathrm{~K}$ by $30 \mathrm{~K}$ (top panel), and from $7 \times 10^{15}$ to by $1.7 \times 10^{17} \mathrm{~cm}^{-2}$ times by $2 \times 10^{16} \mathrm{~cm}^{-2}$ (bottom panel). The white crosses mark the position of the $1.4 \mathrm{~mm}$ continuum emission peaks.

\subsubsection{Velocity field: The rotating toroid}

In Paper I we report on the detection of a clear velocity gradient in both core $\mathrm{A} 1$ and $\mathrm{A} 2$, with $V_{\mathrm{LSR}}$ increasing steadily along a direction at a PA $\sim 50^{\circ}$, which is perpendicular to the axis of the molecular outflow detected toward core A by Furuya et al. (2002). As already discussed in Paper I, the most plausible interpretation for such velocity gradients is that the cores have toroidal structures undergoing rotation about the corresponding outflow axis. In such a scenario, the two cores A1 and A2 would correspond to two distinct rotating toroids. However, the separation of the cores is small, $1^{\prime \prime} .5$ or $12000 \mathrm{AU}$, and we cannot rule out the possibility that they are part of the same geometrically thick rotating toroid, or pipe, elongated along the direction of the outflow. In such a scenario, assuming core A1 as the powering source of the outflow, the velocity gradient detected in the region could be tracing a general rotation of this pipe, related to the rotation associated with the launching of the jet. Taking into account jet models that predict that the flow moves along the magnetic surfaces conserving its angular momentum after the Alfvén surface (e.g. Spruit 1996; Königl \& Pudritz 2000), the jet launching radius should be about half of the radius of the toroid in A1 in order to reach the maximum velocity in A2, which is measured at a distance of $\sim 0.02 \mathrm{pc}$. 


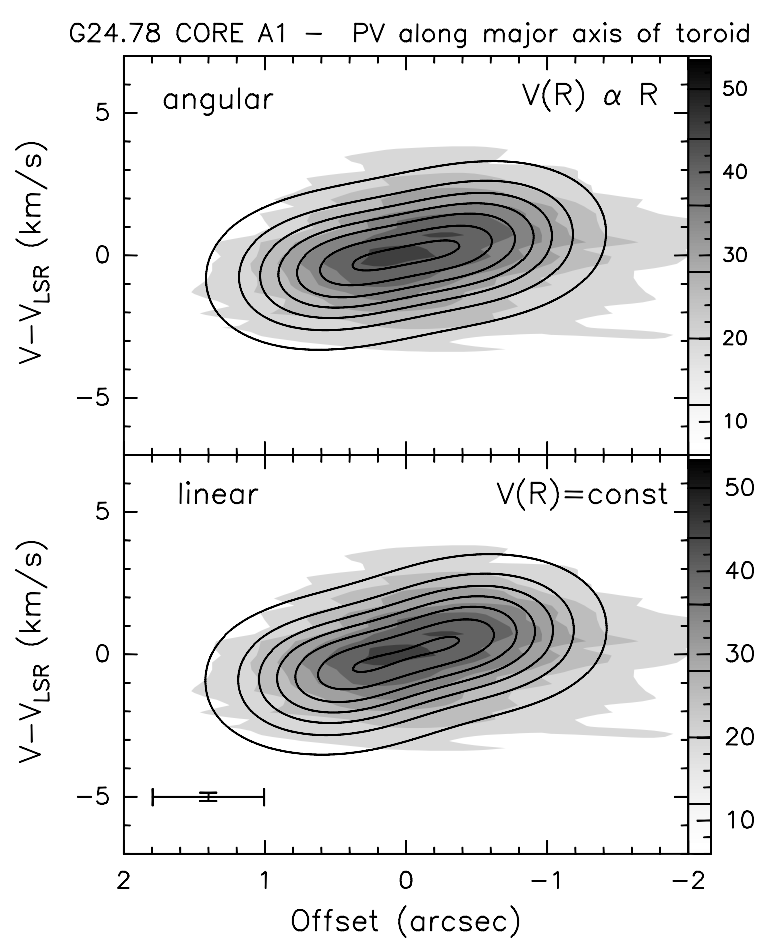

Fig. 22. Same as Fig. 8 along the major axis of the toroid $\left(\mathrm{PA} \sim 50^{\circ}\right)$ in core A1 in G24.78+0.08. The systemic velocity of G24.78+0.08 is $111 \mathrm{~km} \mathrm{~s}^{-1}$. The contour levels are $4,12,20,28,36,44$ and $50 \mathrm{Jy}^{\text {beam }}{ }^{-1}$.

Such a launching radius is consistent with the data, and thus, it calls for further higher angular resolution observations to resolve this question.

Due to the difficulty in establishing which of the two cores, A1 or A2, is powering the bipolar outflow, we arbitrarily assume that it is core A1, as also done in Paper I, because its association with an UC HII region indicates that a YSO has already formed. Thus, we model only the rotating structure associated with core A1. Note that the PV plots for both cores are very similar, and thus, the results and conclusions derived from the modeling, with the exception of the exact value of the model parameters, apply also to core A2. The velocity shift measured over an extent of $\sim 9000$ AU is $\sim 3 \mathrm{~km} \mathrm{~s}^{-1}$, that is, a velocity gradient of about $70 \mathrm{~km} \mathrm{~s}^{-1} \mathrm{pc}^{-1}$. The kinematics of the gas toward core A1 can be investigated with a PV cut of the $\mathrm{CH}_{3} \mathrm{CN}(12-11) \mathrm{K}=3$ emission along a direction $\left(\mathrm{PA} \sim 50^{\circ}\right.$ ) roughly perpendicular to the outflow axis (Fig. 22). As done for G31.41+0.31, we modeled the emission by assuming powerlaw distributions for the temperature and the velocity field in the toroid (see Sect. 3.2.2), and adopted a constant density in the core. The models computed were with constant rotation velocity and with constant angular velocity, because Keplerian rotation is not possible due to the huge mass of the toroid. For the temperature distribution we again adopted a temperature power law $T \propto R^{-3 / 4}$. As can be seen in Fig. 22, from the modeling itself it is not possible to distinguish between constant angular velocity ( $v \propto R$; top panel) or constant rotation $(v=$ const.; bottom panel), because both synthetic PV diagrams fit the data reasonably well. We computed models with and without

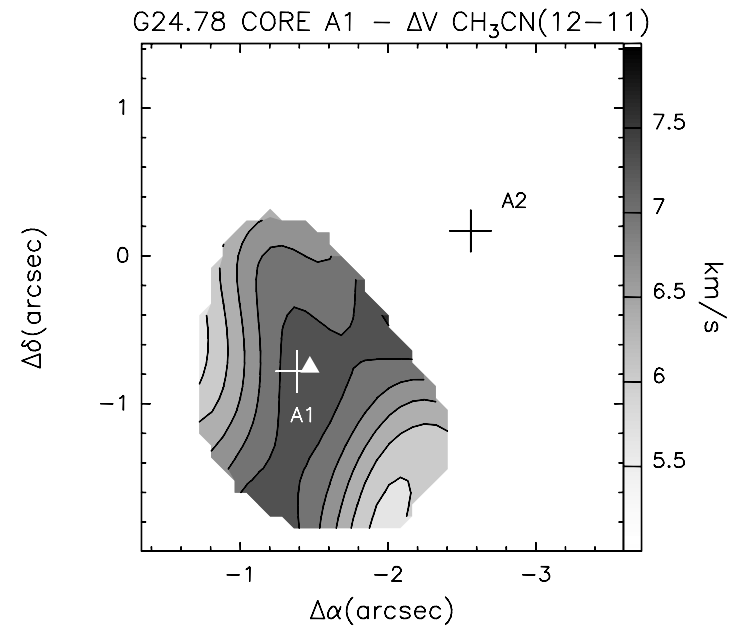

Fig. 23. Line width plot of the $\mathrm{CH}_{3} \mathrm{CN}(12-11)$ toward core $\mathrm{A} 1$ in $\mathrm{G} 24.78+0.08$. The contour levels range from 5.5 to $7.5 \mathrm{~km} \mathrm{~s}^{-1}$ in steps of $0.5 \mathrm{~km} \mathrm{~s}^{-1}$. The crosses mark the positions of the $1.4 \mathrm{~mm}$ continuum emission peaks. The white triangle marks the position of the $1.3 \mathrm{~cm}$ continuum emission peak from the HII region (Codella et al. 1997).

temperature gradients, and both fitted the data equally well. The fact that a molecular outflow and an UC HII region have been detected in association with this core implies the existence of an embedded source powering it. Therefore, although not clearly seen in our data, one may reasonably assume the existence of a temperature gradient driven by the central heating source. Thus, we adopted a temperature distribution $T \propto R^{-3 / 4}$ for the models. The fact that the $\mathrm{G} 24.78+0.08 \mathrm{CH}_{3} \mathrm{CN}$ emission, unlike that of $\mathrm{G} 31.41+0.31$, has no "hole" at the center, is consistent with the gas emission of core A1 in G24.78+0.08 being optically thinner than that of $\mathrm{G} 31.41+0.31$.

The best fit model parameters are given in Table 6 . The value of $R_{\text {inn }}$ is $0 \prime^{\prime} 3(\sim 2300 \mathrm{AU})$, which is the size of the UC HII region at $1.3 \mathrm{~cm}$ at the $3 \sigma$ emission level as recently observed with the VLA in the A configuration (Beltrán, private communication). The value of $v_{\text {rot }}$ is consistent with that derived directly from the velocity gradient in Paper I. The $R_{\text {out }}$ derived from the model is roughly twice the value measured from the $1.4 \mathrm{~mm}$ continuum emission at $50 \%$ of the peak (see Table 1 in Paper I). The value of $N_{\mathrm{CH}_{3} \mathrm{CN}}^{\mathrm{peak}}$ is $\sim 5$ times lower than the column density derived by means of the RD method (see Sect. 4.2.1). As already mentioned for G31.41+0.31, this is due to the fact that the models do not take into account the clumpiness of the region.

The dynamical mass computed with these values assuming that the toroid is seen edge-on by the observer is $M_{\text {dyn }} \sim 34 M_{\odot}$. This value is much smaller than the mass of the core, and, as for $\mathrm{G} 31.41+0.31$, suggests that such a structure is unstable against gravitational collapse. The infall of material onto the embedded millimeter source is also suggested by the line widths, $\Delta V$, measured in the toroid (see Fig. 23). Note that for $\mathrm{G} 24.78+0.08$ the line widths have been measured from $\mathrm{CH}_{3} \mathrm{CN}$, because it has not been possible to properly measure them from $\mathrm{CH}_{3}{ }^{13} \mathrm{CN}$, probably due to an opacity effect. 

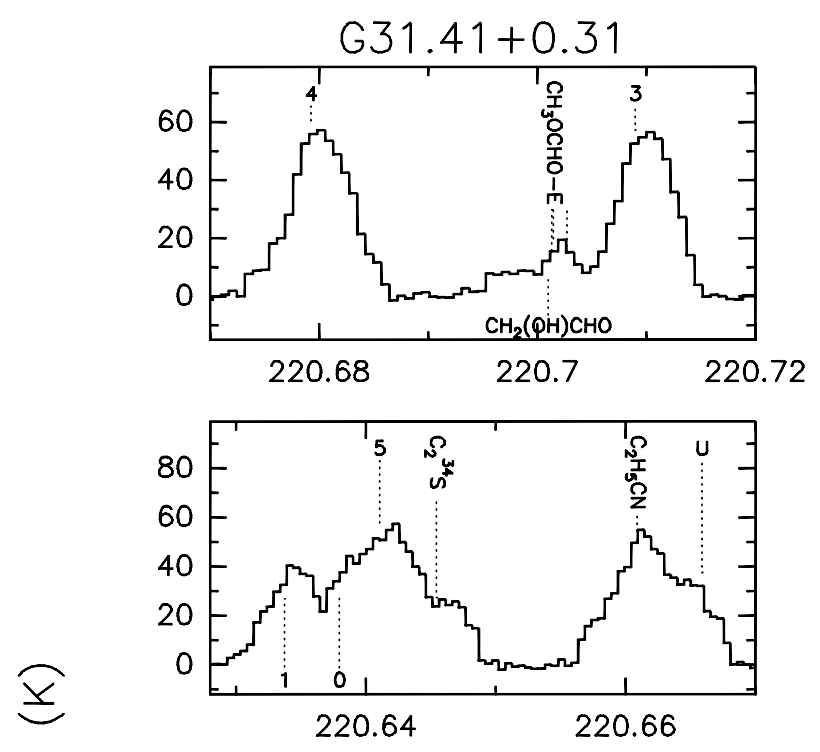

$\stackrel{\infty}{-}$
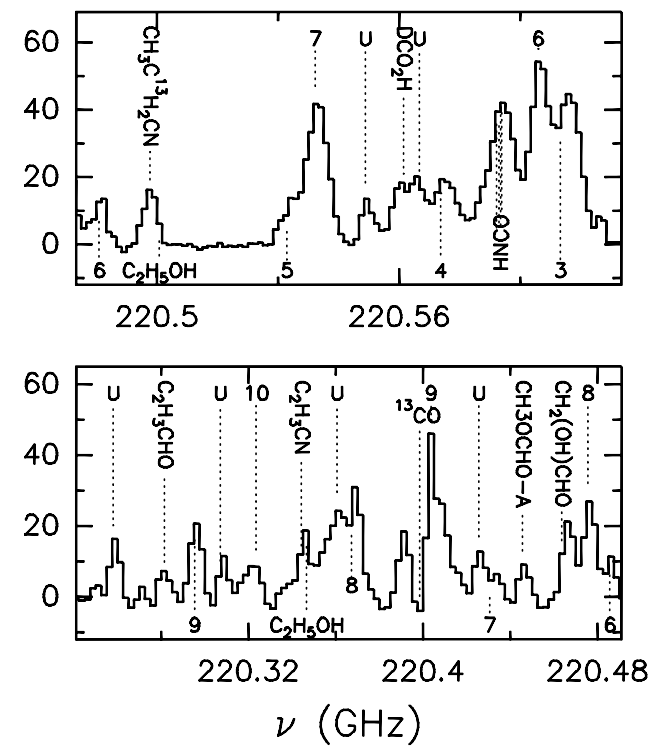

Fig. 24. Serendipitous detections toward the center of G31.41+0.31 obtained with the PdBI. The vertical labels along the profiles point out the identified lines, reported in Tables 11 and 12, and the unidentified peaks, listed in Table 12. The $y$-axis scale is expressed in brightness temperature. The dotted vertical lines are corresponding numbers indicate the position of the $\mathrm{CH}_{3} \mathrm{CN}$ (12-11) $K$-components in the upper part part of each spectra, and of the ${ }^{13} \mathrm{CH}_{3} \mathrm{CN}(12-11) K$-components in the lower part part.

As seen in the figure, $\Delta V$ steadily increases toward the center of the toroid and peaks at the position of the millimeter continuum source. The estimate of $v_{\text {inf }}$ derived from the difference between $\Delta V$ at the position of the $1.4 \mathrm{~mm}$ continuum source and that at the edge of the toroid (see Sect. 3.2.2) is $v_{\text {inf }} \simeq 1.2 \mathrm{~km} \mathrm{~s}^{-1}$ in this case. This value is slightly lower than the $v_{\text {rot }}$ obtained from the models or from the velocity gradient in the toroid. The accretion rate derived for such a $v_{\text {inf }}$ is $\dot{M}_{\text {acc }} \sim 9 \times 10^{-3} M_{\odot} \mathrm{yr}^{-1}$. Such a huge accretion rate supports
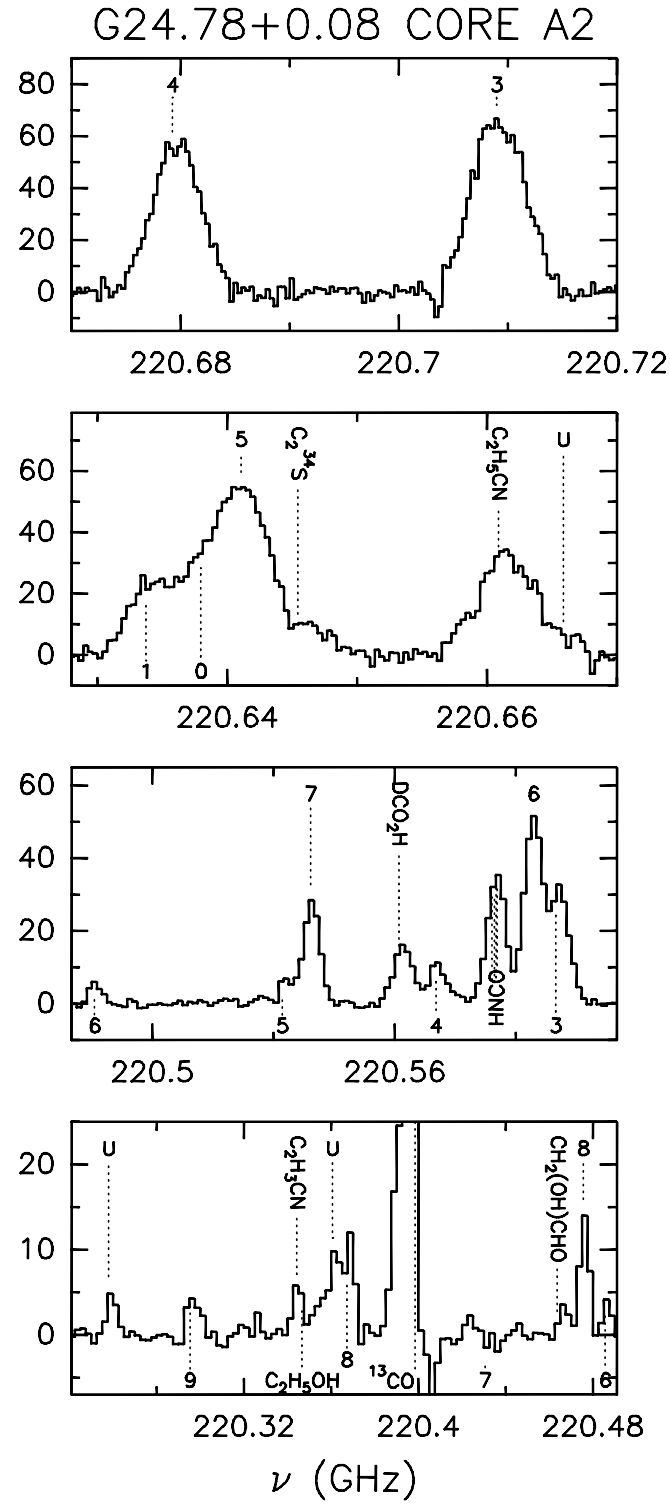

Fig. 25. Serendipitous detections toward the peak position of core A2 in G24.78+0.08 obtained with the PdBI. The vertical labels along the profiles point out the identified lines, reported in Tables 11 and 12, and the unidentified peaks, listed in Table 12. The $y$-axis scale is expressed in brightness temperature. The dotted vertical lines are corresponding numbers indicate the position of the $\mathrm{CH}_{3} \mathrm{CN}$ (12-11) $K$-components in the upper part part of each spectra, and of the ${ }^{13} \mathrm{CH}_{3} \mathrm{CN}(12-11)$ $K$-components in the lower part part.

the disk-accretion scenario also for the formation of the massive YSO embedded in core A1 in G24.78+0.08.

\section{Other molecules in $\mathrm{G} 31.41+0.31$ and G24.78+0.08}

Figures 24 and 25 show some portions of the spectra obtained toward G31.41+0.31 and core A2 in G24.78+0.08, respectively. Taking into account that the spectra for both core A1 and A2 in G24.78+0.08 are quite similar, we have only plotted the spectra toward A2, where the detections are 
Table 11. Serendipitous detections toward G31.41+0.31 and G24.78+0.08 (A1 and A2) ${ }^{a}$.

\begin{tabular}{|c|c|c|c|c|c|c|c|}
\hline Molecule & Transition & $\begin{array}{c}\text { Rest frequency } \\
(\mathrm{MHz})\end{array}$ & $\begin{array}{l}E_{\mathrm{u}} \\
(\mathrm{K})\end{array}$ & $\begin{array}{c}V_{\mathrm{LSR}} \\
\left(\mathrm{km} \mathrm{s}^{-1}\right)\end{array}$ & $\begin{array}{c}F W H M \\
\left(\mathrm{~km} \mathrm{~s}^{-1}\right)\end{array}$ & $\begin{array}{l}T_{\mathrm{B}} \\
(\mathrm{K})\end{array}$ & $\begin{array}{c}\int T_{\mathrm{B}} \mathrm{d} V \\
\left(\mathrm{~K} \mathrm{~km} \mathrm{~s}^{-1}\right)\end{array}$ \\
\hline \multicolumn{8}{|c|}{ G31.41+0.31 } \\
\hline $\mathrm{C}_{2} \mathrm{H}_{5} \mathrm{CN}^{b}$ & $\left(25_{2,24}-24_{2,23}\right)$ & 220660.92 & 143 & $96.3 \pm 0.1$ & $7.7 \pm 0.2$ & $50.6 \pm 3.3$ & $415 \pm 12$ \\
\hline $\mathrm{C}_{2}{ }^{34} \mathrm{~S}^{c}$ & $\left(24_{23}-23_{23}\right)$ & 220645.47 & 187 & $96.1 \pm 0.5$ & $7.2 \pm 0.5$ & $17.4 \pm 4.2$ & $133 \pm 15$ \\
\hline $\mathrm{HNCO}^{d}$ & $\left(10_{1,9}-9_{1,8}\right)$ & $220585.20^{e}$ & 102 & $96.6 \pm 0.4$ & $12.2 \pm 1.2^{e}$ & $39.7 \pm 4.3$ & $518 \pm 39$ \\
\hline $\mathrm{DCO}_{2} \mathrm{H}^{f}$ & $\left(10_{6,4}-9_{6,3}\right)$ & $220561.16^{g}$ & 139 & $98.4 \pm 0.5$ & $6.4 \pm 1.5$ & $14.4 \pm 2.1$ & $98 \pm 44$ \\
\hline $\mathrm{CH}_{2}(\mathrm{OH}) \mathrm{CHO}^{h}$ & $\left(20_{2,18}-19_{3,17}\right)$ & 220463.87 & 120 & $93.1 \pm 0.8^{h}$ & $8.2 \pm 1.8$ & $22.1 \pm 3.7$ & $193 \pm 36$ \\
\hline $\mathrm{CH}_{3} \mathrm{OCHO}-\mathrm{A}$ & $\left(25_{11,15}-26_{9,18}\right)$ & 220445.79 & 272 & $96.0 \pm 1.1$ & $7.9 \pm 2.4$ & $10.9 \pm 3.4$ & $92 \pm 26$ \\
\hline \multicolumn{8}{|c|}{ G24.78+0.08 core A1 } \\
\hline $\mathrm{C}_{2} \mathrm{H}_{5} \mathrm{CN}^{b}$ & $\left(25_{2,24}-24_{2,23}\right)$ & 220660.92 & 143 & $111.4 \pm 0.1$ & $4.3 \pm 0.2$ & $24.3 \pm 2.0$ & $111 \pm 5$ \\
\hline $\mathrm{HNCO}^{d}$ & $\left(10_{1,9}-9_{1,8}\right)$ & $220585.20^{e}$ & 102 & $111.8 \pm 1.7$ & $7.0 \pm 1.7^{e}$ & $19.9 \pm 1.7$ & $148 \pm 28$ \\
\hline $\mathrm{DCO}_{2} \mathrm{H}^{f}$ & $\left(10_{6,4}-9_{6,3}\right)$ & $220561.16^{g}$ & 139 & $111.7 \pm 0.2$ & $3.9 \pm 0.7$ & $8.9 \pm 1.1$ & $37 \pm 5$ \\
\hline $\mathrm{CH}_{2}(\mathrm{OH}) \mathrm{CHO}^{h}$ & $\left(20_{2,18}-19_{3,17}\right)$ & 220463.87 & 120 & $-^{i}$ & $-^{i}$ & $3.0 \pm 0.5^{i}$ & $41 \pm 3^{i}$ \\
\hline \multicolumn{8}{|c|}{ G24.78+0.08 core A2 } \\
\hline $\mathrm{C}_{2} \mathrm{H}_{5} \mathrm{CN}^{b}$ & $\left(25_{2,24}-24_{2,23}\right)$ & 220660.92 & 143 & $110.4 \pm 0.1$ & $6.7 \pm 0.4$ & $33.4 \pm 1.4$ & $237 \pm 11$ \\
\hline $\mathrm{C}_{2}{ }^{34} \mathrm{~S}^{c}$ & $\left(24_{23}-23_{23}\right)$ & 220645.47 & 187 & $110.5 \pm 0.6$ & $6.1 \pm 1.0$ & $10.7 \pm 1.6$ & $70 \pm 13$ \\
\hline $\mathrm{HNCO}^{d}$ & $\left(10_{1,9}-9_{1,8}\right)$ & $220585.20^{e}$ & 102 & $110.9 \pm 0.1$ & $7.5 \pm 1.2^{e}$ & $35.3 \pm 0.8$ & $281 \pm 5$ \\
\hline $\mathrm{DCO}_{2} \mathrm{H}^{f}$ & $\left(10_{6,4}-9_{6,3}\right)$ & $220561.16^{g}$ & 139 & $109.7 \pm 0.2$ & $7.2 \pm 0.4$ & $16.1 \pm 1.1$ & $124 \pm 7$ \\
\hline $\mathrm{CH}_{2}(\mathrm{OH}) \mathrm{CHO}^{h}$ & $\left(20_{2,18}-19_{3,17}\right)$ & 220463.87 & 120 & $107.1 \pm 0.3^{i}$ & $6.4 \pm 0.7$ & $3.9 \pm 0.4$ & $26 \pm 3$ \\
\hline
\end{tabular}

${ }^{a}$ Spectra taken toward the peak positions of G31.41+0.31 and cores A1 and A2 in G24.78+0.08.

${ }^{b}$ Blended with the 220665.91 unidentified line (see Table 13).

${ }^{c}$ Blended with the $K=5$ component of $\mathrm{CH}_{3} \mathrm{CN}$ (12-11).

${ }^{d}$ Blended with the $K=6$ and $K=4$ components of $\mathrm{CH}_{3} \mathrm{CN}$ and ${ }^{13} \mathrm{CH}_{3} \mathrm{CN}(12-11)$, respectively.

${ }^{e}$ The HNCO $\left(10_{1,9}-9_{1,8}\right)$ line splits into six hyperfine components with $\Delta F=0, \pm 1$ spread over $0.6 \mathrm{MHz}$ and thus not resolved with the present spectral resolution. The frequency refers to the $F-F^{\prime}=10-9$ line.

${ }^{f}$ This line could be blended with the high excitation $\left(E_{\mathrm{u}}=625 \mathrm{~K}\right) \mathrm{C}_{2} \mathrm{H}_{3} \mathrm{CN}\left(10_{3,7}-10_{2,8}\right)$ line at $220651.39 \mathrm{MHz}$.

${ }^{g}$ The rest frequency corresponds also to the $\mathrm{DCO}_{2} \mathrm{H}\left(10_{6,5}-9_{6,4}\right)$ line, which has the same excitation.

${ }^{h}$ According to the databases for molecular spectroscopy, glycolaldehyde results to be the most probable candidate to identify the present emission at this frequency. If confirmed, this would be the first detection of $\mathrm{CH}_{2}(\mathrm{OH}) \mathrm{CHO}$ outside the Galactic center (see text).

${ }^{i}$ Bad fit because of non-Gaussian profile.

more clearly visible. In these figures one can see that besides the expected $\mathrm{CH}_{3} \mathrm{CN}$ and ${ }^{13} \mathrm{CH}_{3} \mathrm{CN}$ patterns, numerous other lines have been serendipitously detected. These lines have been identified, when possible, by using the Jet Propulsion Laboratory, Cologne, and Lovas databases for molecular spectroscopy. Emission due to $\mathrm{C}_{2} \mathrm{H}_{5} \mathrm{CN}, \mathrm{C}_{2}{ }^{34} \mathrm{~S}, \mathrm{HNCO}, \mathrm{DCO}_{2} \mathrm{H}$, and $\mathrm{CH}_{3} \mathrm{OCHO}-\mathrm{A}$ high excitation $(100-270 \mathrm{~K})$ transitions have been measured. For these lines, Table 11 lists the transition, the rest frequency, the upper level excitation $E_{\mathrm{u}}(\mathrm{K})$, and line parameters obtained with a Gaussian fit, i.e. the velocity $V_{\mathrm{LSR}}\left(\mathrm{km} \mathrm{s}^{-1}\right)$, the $F W H M$ line width $\left(\mathrm{km} \mathrm{s}^{-1}\right)$, the peak brightness temperature $T_{\mathrm{B}}(\mathrm{K})$, and the integral under the line $\int T_{\mathrm{B}} \mathrm{d} V\left(\mathrm{~K} \mathrm{~km} \mathrm{~s}^{-1}\right)$.

A special case is represented by a peak at $220.46 \mathrm{GHz}$ (see Table 11), where the only reasonable candidate to our knowledge is the transition $J_{\mathrm{K}_{-} \mathrm{K}_{+}}=20_{2,18}-19_{3,17}$ of glycolaldehyde $\left(\mathrm{CH}_{2}(\mathrm{OH}) \mathrm{CHO}\right)$, whose excitation $(120 \mathrm{~K})$ well fits with the temperature measured toward G31.41+0.31 and $\mathrm{G} 24.78+0.08$. Glycolaldehyde is an isomer to methyl formate $\left(\mathrm{CH}_{3} \mathrm{OCHO}\right)$, here detected at $220.45 \mathrm{GHz}$. Whereas the detection of $\mathrm{CH}_{3} \mathrm{OCHO}$ is not surprising since it is a common species in hot cores (e.g. van Dishoeck \& Blake 1998),
$\mathrm{CH}_{2}(\mathrm{OH}) \mathrm{CHO}$ has been detected up to date only toward Sagittarius B2N (Hollis et al. 2000). The reasons could be that complex interstellar molecules seem to prefer the $\mathrm{C}-\mathrm{O}-\mathrm{C}$ backbones with respect to the $\mathrm{C}-\mathrm{C}-\mathrm{O}$ ones (see Fig. 2 of Hollis et al. 2000). Note that other $\mathrm{CH}_{2}(\mathrm{OH}) \mathrm{CHO}$ lines lie inside the spectral windows here investigated, but unfortunately they are closely blended with other bright lines. In conclusion, we can only claim a tentative detection of $\mathrm{CH}_{2}(\mathrm{OH}) \mathrm{CHO}$ toward $\mathrm{G} 31.41+0.31$ and G24.78+0.08, which calls for further observations to confirm the occurrence of glycolaldehyde emission in hot cores. Note that there is a difference between the line rest frequency and the frequency of the observed peak $(\sim 2 \mathrm{MHz})$, as indicated by a slightly different peak velocity from the $V_{\mathrm{LSR}}$ of the region (Table 11). If the detection of glycolaldehyde is confirmed, this would imply that the rest frequency listed in the spectral catalogues should be refined.

Table 12 reports the observed frequency, the corresponding spectral resolution and the line candidates with their frequency and excitation for those spectral features that could not be unambiguously identified. The candidate transitions have frequencies that fall inside the spectral features, and were selected on the basis of the following criteria: (i) emission lines 
Table 12. Other molecular tracers detected toward G31.41+0.31 and G24.78+0.08.

\begin{tabular}{|c|c|c|c|c|c|}
\hline \multirow{2}{*}{$\begin{array}{c}\text { Obs. frequency } \\
(\mathrm{MHz})\end{array}$} & \multirow{2}{*}{$\begin{array}{c}\mathrm{d} v \\
(\mathrm{MHz}) \\
\end{array}$} & \multirow[t]{2}{*}{ Source } & \multicolumn{3}{|c|}{ Line candidates } \\
\hline & & & Transition & Frequency $(\mathrm{MHz})$ & $E_{\mathrm{u}}(\mathrm{K})$ \\
\hline \multicolumn{6}{|c|}{ Blended lines } \\
\hline \multirow[t]{4}{*}{$220702.17 \pm 0.15^{b}$} & 0.7 & G31 & $\mathrm{CH}_{3}$ OCHO-E $\left(10_{4,7}-9_{3,7}\right)$ & 220702.75 & 43 \\
\hline & & & $\mathrm{CH}_{3} \mathrm{OCHO}-\mathrm{E}\left(24_{3,22}-24_{2,23}\right)$ & 220701.52 & 169 \\
\hline & & & $\mathrm{CH}_{3} \mathrm{OCHO}-\mathrm{E}\left(24_{3,22}-24_{1,24}\right)$ & 220701.36 & 169 \\
\hline & & & $\mathrm{CH}_{2}(\mathrm{OH}) \mathrm{CHO}\left(40_{9,32}-40_{8,33}\right)$ & 220701.03 & 511 \\
\hline \multirow[t]{2}{*}{$220498.34 \pm 0.08^{c}$} & 1.3 & G31 & $\mathrm{C}_{2} \mathrm{H}_{5} \mathrm{OH}\left(19_{5,14}-19_{4,15}\right)$ & 220500.70 & 250 \\
\hline & & & $\mathrm{CH}_{3}^{13} \mathrm{CH}_{2} \mathrm{CN}\left(7_{4,3}-6_{3,4}\right)$ & 220498.40 & 29 \\
\hline \multirow[t]{2}{*}{$220345.51 \pm 0.16$} & 2.5 & G31, G24 & $\mathrm{C}_{2} \mathrm{H}_{5} \mathrm{OH}\left(7_{3,4}-7_{1,7}\right)$ & 220346.86 & 91 \\
\hline & & & $\mathrm{C}_{2} \mathrm{H}_{3} \mathrm{CN}\left(9_{4,5}-10_{3,8}\right)$ & 220344.45 & 55 \\
\hline
\end{tabular}

\footnotetext{
${ }^{a}$ The observed frequencies have been measured from the spectra toward G31.41+0.31 due to a better signal-to-noise ratio of the profiles.

${ }^{b}$ Blended with a non Gaussian profile, which produces a plateau in the observed profiles (see Fig. 24). A possible candidate for the emission is $\mathrm{C}_{2} \mathrm{H}_{3} \mathrm{CN}^{\mathrm{b}}\left(10_{3,7}-10_{2,8}\right)$ at a frequency of $220695.42 \mathrm{MHz}$.

${ }^{c}$ This line could be blended with the very high excitation $\left(E_{\mathrm{u}}=1005 \mathrm{~K}\right) \mathrm{CH}_{3} \mathrm{OCHO}-\mathrm{E}\left(46_{0,46}-45_{4,41}\right)$ line at $220667.14 \mathrm{MHz}$.
}

Table 13. Unidentified lines detected toward G31.41+0.31 and G24.78+0.08.

\begin{tabular}{ccc}
\hline \hline $\begin{array}{c}\text { Frequency }^{a} \\
(\mathrm{MHz})\end{array}$ & $\begin{array}{c}\text { Spectral resolution } \\
(\mathrm{MHz})\end{array}$ & Source \\
\hline $220665.91 \pm 0.15$ & 2.5 & $\mathrm{G} 31, \mathrm{G} 24$ \\
$220564.07 \pm 0.29$ & 1.3 & $\mathrm{G} 31$ \\
$220551.97 \pm 0.41$ & 1.3 & $\mathrm{G} 31$ \\
$220426.40 \pm 0.18$ & 2.5 & $\mathrm{G} 31$ \\
$220361.37 \pm 0.50$ & 2.5 & $\mathrm{G} 31, \mathrm{G} 24$ \\
$220308.68 \pm 0.43$ & 2.5 & $\mathrm{G} 31$ \\
$220258.91 \pm 0.31$ & 2.5 & $\mathrm{G} 31$ \\
\hline
\end{tabular}

a The frequencies have been measured from the spectra toward G31.41+0.31, due to a better signal-to-noise ratio of the profiles, assuming a systemic velocity $V_{\mathrm{LSR}}=97 \mathrm{~km} \mathrm{~s}^{-1}$.

known to be hot core tracers, such as $\mathrm{CH}_{3} \mathrm{OCHO}-\mathrm{E}, \mathrm{C}_{2} \mathrm{H}_{3} \mathrm{CN}$, and $\mathrm{C}_{2} \mathrm{H}_{5} \mathrm{OH}$ (e.g. van Dishoeck \& Blake 1998; Cazaux et al. 2003); and (ii) lines with excitations in agreement with the temperatures measured toward G31.41+0.31 and G24.78+0.08 $\left(E_{\mathrm{u}} \lesssim 500 \mathrm{~K}\right)$. Finally, Table 13 reports the peak frequencies and the relative spectral resolution of the unidentified lines toward $\mathrm{G} 31.41+0.31$ and $\mathrm{G} 24.78+0.08$. In conclusion, the gas associated with $\mathrm{G} 31.41+0.31$ results more chemically rich than that in $\mathrm{G} 24.78+0.08$ : this could be caused by a different evolutionary stage or it could simply be a luminosity effect being $\mathrm{G} 31.41+0.31$ more luminous than $\mathrm{G} 24.78+0.08$.

In order to understand which regions are traced by these complex molecules, the maps of the emission due to the unblended lines is shown in Figs. 26 (for G31.41+0.31) and 27 (for $\mathrm{G} 24.78+0.08$ ). The distributions of $\mathrm{C}_{2} \mathrm{H}_{5} \mathrm{CN}$, $\mathrm{HNCO}$, $\mathrm{CH}_{3} \mathrm{OCHO}-\mathrm{A}$, and $\mathrm{CH}_{2}(\mathrm{OH}) \mathrm{CHO}$ (greyscale) have been compared with that of the $K=0,1$, and 2 components of $\mathrm{CH}_{3} \mathrm{CN}$ (contours). For G31.41+0.31, it is possible to see that the emission due to these molecules comes from the central region where the protostars are located as indicated by the positions of the $1.4 \mathrm{~mm}$ (white cross) and $7 \mathrm{~mm}$ (white dots) peaks. This result confirms the presence of a hot molecular core associated with G31.41+0.31, in agreement with the increase of temperature and density toward the center of the region as measured with $\mathrm{CH}_{3} \mathrm{CN}$ (see Fig. 7). Moreover, Fig. 26 shows that the emission from different tracers peaks at slightly different positions, indicating that $\mathrm{C}_{2} \mathrm{H}_{5} \mathrm{CN}$, $\mathrm{HNCO}, \mathrm{CH}_{3} \mathrm{OCHO}-$ $\mathrm{A}$, and $\mathrm{CH}_{2}(\mathrm{OH}) \mathrm{CHO}$ are tracing different portions of the hot core. Figure 27 shows a similar scenario for $\mathrm{G} 24.78+0.08$ : the $\mathrm{C}_{2} \mathrm{H}_{5} \mathrm{CN}, \mathrm{HNCO}$, and $\mathrm{CH}_{2}(\mathrm{OH}) \mathrm{CHO}\left(\mathrm{CH}_{3} \mathrm{OCHO}-\mathrm{A}\right.$ has not been detected toward $\mathrm{G} 24.78+0.08$ ) emission traces the two hot cores, A1 and A2, confirming that each of them is associated with a distinct site of star formation and is hosting YSOs.

\section{Conclusions}

We have analyzed the millimeter high angular resolution data, obtained with the BIMA and IRAM PdBI interferometers, of the dust and gas emission toward the high-mass star forming regions $\mathrm{G} 31.41+0.31$ and $\mathrm{G} 24.78+0.08$. The aim was a thorough study of the structure and kinematics of the rotating toroids detected in both hot cores in Paper I.

The $\mathrm{CH}_{3} \mathrm{CN}$ (12-11) emission of the toroids in $\mathrm{G} 31.41+0.31$ and core A1 in $\mathrm{G} 24.78+0.08$ has been modeled assuming that it arises from a disk-like structure seen edge-on, with a radial velocity field. For G31.41+0.31 the model properly fits the data for a velocity $v_{\text {rot }} \simeq 1.7 \mathrm{~km} \mathrm{~s}^{-1}$ at the outer radius $R_{\text {out }} \simeq 13400 \mathrm{AU}$ and an inner radius $R_{\text {inn }} \simeq 1340 \mathrm{AU}$, while for core $\mathrm{A} 1$ in $\mathrm{G} 24.78+0.08$ the best fit is obtained for $v_{\text {rot }} \simeq 2.0 \mathrm{~km} \mathrm{~s}^{-1}$ at $R_{\text {out }} \simeq 7700 \mathrm{AU}$ and $R_{\text {inn }} \simeq 2300 \mathrm{AU}$. Unlike the Keplerian disks detected around less luminous stars such as IRAS 20126+4104 (e.g. Cesaroni et al. 1997), NGC 7538S (Sandell et al. 2003), or M 17 (Chini et al. 2004), these toroids are not undergoing Keplerian rotation. From the modeling itself, however, it is not possible to distinguish between constant rotation or constant angular velocity, since both velocity fields suitably fit the data. The best fit models have been computed assuming for the toroids a temperature gradient of the type $T \propto R^{-3 / 4}$, typical of accretion circumstellar disks (Natta 2000), with a temperature at the outer radius $T_{\text {out }} \simeq 100 \mathrm{~K}$ for both cores. 

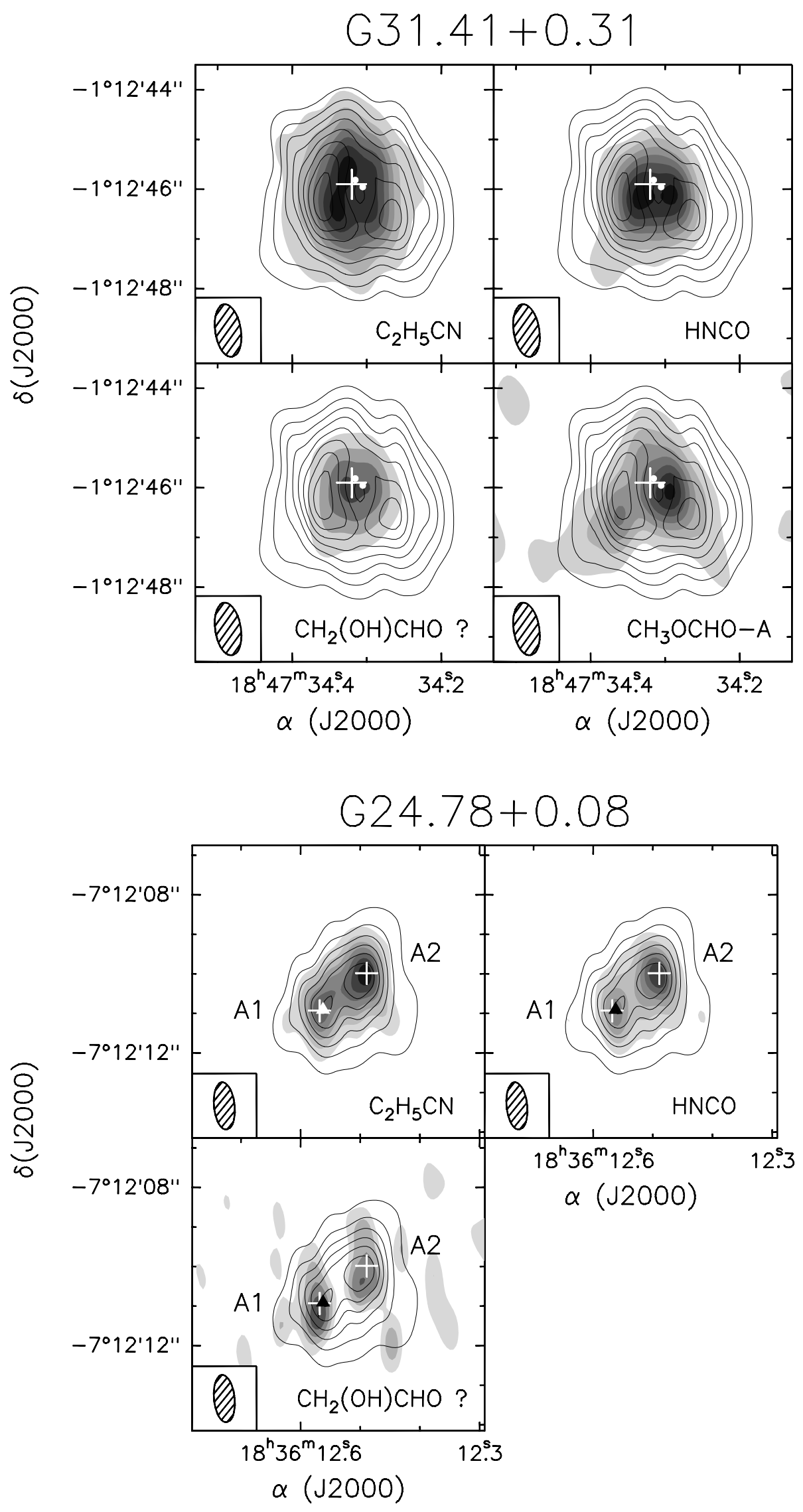

Fig. 26. Overlay of the $\mathrm{PdBI} \mathrm{CH}_{3} \mathrm{CN}$ (12-11) emission averaged under the $K=$ 0,1 , and 2 components (contours) and the $\mathrm{C}_{2} \mathrm{H}_{5} \mathrm{CN}\left(25_{2,24}-24_{2,23}\right)$ emission (top left), the HNCO $\left(10_{1,9}-9_{1,8}\right)$ emission (top right), the $\mathrm{CH}_{2}(\mathrm{OH}) \mathrm{CHO}\left(20_{2,18}-19_{3,17}\right)$ emission (bottom left), and the $\mathrm{CH}_{3} \mathrm{OCHO}$ A $\left(25_{11,15}-26_{9,18}\right)$ emission (bottom right) toward $\mathrm{G} 31.41+0.31$. Contours are the same as in Fig. 3. Greyscale levels range from 0.10 to $1.18 \mathrm{Jy}^{-1}$ beam $^{-1}$ in steps of $0.18 \mathrm{Jy}^{\text {beam }}{ }^{-1}$ for $\mathrm{C}_{2} \mathrm{H}_{5} \mathrm{CN}$, from 0.10 to $0.94 \mathrm{Jy}^{\text {beam }^{-1}}$ in steps of $0.14 \mathrm{Jy}^{\mathrm{beam}}{ }^{-1}$ for $\mathrm{HNCO}$, from 0.10 to $0.46 \mathrm{Jy} \mathrm{beam}^{-1}$ in steps of $0.12 \mathrm{Jy} \mathrm{beam}^{-1}$ for $\mathrm{CH}_{2}(\mathrm{OH}) \mathrm{CHO}$, and from 0.01 to $0.13 \mathrm{Jy}^{-1}$ beam $^{-1}$ in steps of $0.02 \mathrm{Jy} \mathrm{beam}^{-1}$ for $\mathrm{CH}_{3} \mathrm{OCHO}-\mathrm{A}$. The synthesized beam is shown in the lower lefthand corner. The white cross marks the position of the $1.4 \mathrm{~mm}$ continuum emission peak, and the white dots the $7 \mathrm{~mm}$ continuum emission peaks detected by Hofner (private communication).

Fig. 27. Overlay of the PdBI $\mathrm{CH}_{3} \mathrm{CN}$ (12-11) emission averaged under the $K=$ 0,1 , and 2 components (contours) and the $\mathrm{C}_{2} \mathrm{H}_{5} \mathrm{CN}\left(25_{2,24}-24_{2,23}\right)$ emission (top left), the HNCO $\left(10_{1,9}-9_{1,8}\right)$ emission (top right), and the $\mathrm{CH}_{2}(\mathrm{OH}) \mathrm{CHO}\left(20_{2,18}-\right.$ $19_{3,17}$ ) emission (bottom left) toward cores $\mathrm{A} 1$ and $\mathrm{A} 2$ in G24.78+0.08. Contours levels range from 0.10 to $1.00 \mathrm{Jy}_{\text {beam }^{-1}}$ in steps of $0.18 \mathrm{Jy} \mathrm{beam}^{-1}$. Greyscale levels range from 0.10 to $0.70 \mathrm{Jy}^{b^{-1}}$ in steps of $0.10 \mathrm{Jy} \mathrm{beam}^{-1}$ for $\mathrm{C}_{2} \mathrm{H}_{5} \mathrm{CN}$, from 0.10 to $0.60 \mathrm{Jy} \mathrm{beam}^{-1}$ in steps of $0.10 \mathrm{Jy} \mathrm{beam}^{-1}$ for $\mathrm{HNCO}$, and from 0.02 to $0.10 \mathrm{Jy} \mathrm{beam}^{-1}$ in steps of $0.02 \mathrm{Jy}$ beam $^{-1}$ for $\mathrm{CH}_{2}(\mathrm{OH}) \mathrm{CHO}$. The synthesized beam is shown in the lower left-hand corner. The white crosses denote the position of the $1.4 \mathrm{~mm}$ continuum emission peaks. The triangle marks the position of the VLA $1.3 \mathrm{~cm}$ continuum emission peak (Codella et al. 1997). 
The $M_{\mathrm{dyn}}$ needed for equilibrium derived from the models is much smaller than the mass of the cores. This suggests, as already pointed out in Paper I, that such toroids are unstable and undergoing gravitational collapse. The collapse is supported also by the fact that the $\mathrm{CH}_{3}{ }^{13} \mathrm{CN}$ or $\mathrm{CH}_{3} \mathrm{CN}$ line width measured in the cores increases toward the center of the toroids in G31.41+0.31 and G24.78+0.08 A1. The estimates of $v_{\text {inf }}$ and $\dot{M}_{\text {acc }}$ are $\sim 2 \mathrm{~km} \mathrm{~s}^{-1}$ and $\sim 3 \times 10^{-2} M_{\odot} \mathrm{yr}^{-1}$ for $\mathrm{G} 31.41+0.31$, and $\sim 1.2 \mathrm{~km} \mathrm{~s}^{-1}$ and $\sim 9 \times 10^{-3} M_{\odot} \mathrm{yr}^{-1}$ for G24.78+0.08 A1. Accretion rates that large could weaken the effect of stellar winds and radiation pressure thus allowing further accretion on the star. This would support theories according to which highmass stars form like their lower mass counterparts through nonspherical accretion with large accretion rates.

The values of $T_{\text {rot }}$ and $N_{\mathrm{CH}_{3} \mathrm{CN}}$, derived by means of the RD method, for both G31.41+0.31 and the sum of cores A1 and A2 (core A of Codella et al. 1997) in G24.78+0.08 are in the range $132-164 \mathrm{~K}$ and $2-8 \times 10^{16} \mathrm{~cm}^{-2}$.

For G31.41+0.31, the most plausible explanation for the apparent toroidal morphology seen in the lower $K$ transitions of $\mathrm{CH}_{3} \mathrm{CN}(12-11)$ in Paper I is self-absorption, which is caused by the high optical depth and the existence of a temperature gradient in the core. Indeed, the $N_{\mathrm{CH}_{3} \mathrm{CN}}$ presents a peak toward the apparent dip in the $\mathrm{CH}_{3} \mathrm{CN}$ brightness temperature map.

Acknowledgements. We would like to thank Francesca Bacciotti, Daniele Galli and Malcolm Walmsley for helpful discussions and suggestions.

\section{References}

Adams, F. C., Lada, C. J., \& Shu, F. H. 1987, ApJ, 312, 788

Allen, A., Li, Z.-Y., \& Shu, F. H. 2003, ApJ, 599, 363

Beltrán, M. T., Cesaroni, R., Neri, R., et al. 2004, ApJ, 601, L187 (Paper I)

Bergin, E. A., \& Langer, W. D. 1997, ApJ, 486, 316

Bonnell, I. A., Bate, M. R., \& Zinnecker, H. 1998, MNRAS, 298, 93

Cazaux, S., Tielens, A. G. G. M., Ceccarelli, C., et al. 2003, ApJ, 593, L51

Cesaroni, R., Olmi, L., Walmsley, C. M., Churchwell, E., \& Hofner, P. 1994, ApJ, 435, L137

Cesaroni, R., Felli, M., Testi, L., Walmsley, C. M., \& Olmi, L. 1997, A\&A, 435, L137

Cesaroni, R., Hofner, P., Walmsley, C. M., \& Churchwell, E. 1998, A\&A, 331, 709
Cesaroni, R., Felli, M., Jenness, T., et al. 1999, A\&A, 345, 949

Cesaroni, R. 2002, Highlights of Astronomy, 12, 156

Cesaroni, R., Codella, C., Furuya, R. S., \& Testi, L. 2003, A\&A, 401, 227

Chini, R., Hoffmeister, V., Kimeswenger, S., et al. 2004, Nature, 429, 155

Codella, C., Testi, L., \& Cesaroni, R. 1997, A\&A, 325, 282

Condon, J. J., Cotton, W. D., Greisen, E. W., et al. 1998, AJ, 115, 1693

Crutcher, R. M. 1999, ApJ, 520, 706

Fontani, F., Cesaroni, R., Caselli, P., \& Olmi, L. 2002, A\&A, 389, 603

Furuya, R. S., Cesaroni, R., Codella, C., et al. 2002, A\&A, 390, L1

Gibb, A. G., Wyrowski, F., \& Mundy, L. G. 2004, ApJ, 616, 301

Hatchell, J., Fuller, G. A., Millar, T. J., Thompson, M. A., \& Macdonald, G. H. 2000, A\&A, 357, 637

Hollis, J. M., Lovas, F. J., \& Jewell, P. R. 2000, ApJ, 540, L107

Jijina, J., \& Adams, F. C. 1996, ApJ, 462, 874

Königl, A., \& Pudritz, R. 2000, in Protostars and Planets, IV, ed. V. Mannings, A. P. Boss, \& S. S. Russell (Tucson: Univ. Arizona Press), 759

Lovas, F. J. 1992, J. Phys. Chem. Ref. Data, 21, 181

Maxia., C., Testi, L., Cesaroni, R., \& Walmsley, C. M. 2001, A\&A, 371,287

Molinari, S., Testi, L., Brand, J., Cesaroni, R., \& Palla, F. 1998, ApJ, 505, L39

Nakano, T., Hasegawa, T., \& Norman, C. 1995, ApJ, 450, 183

Natta, A. 2000, in Infrared space astronomy, today and tomorrow, ed. F. Casoli, J. Lequeux, \& F. David, Les Houches Summer School, 70, 193

Ohashi, N., Hayashi, M., Ho, P. T. P., et al. 1997, ApJ, 488, 317

Olmi, L., Cesaroni, R., \& Walmsley, C. M. 1993, A\&A, 276, 489

Olmi, L., Cesaroni, R., Neri, R., \& Walmsley, C. M. 1996a, A\&A, 315,565

Olmi, L., Cesaroni, R., \& Walmsley, C. M. 1996b, A\&A, 307, 599

Palla, F., \& Stahler, S. W. 1993, ApJ, 418, 414

Sandell, G., Wright, M., Forster, J. R. 2003, ApJ, 590, L45

Simon, M., Dutrey, A., \& Guilloteau, S. 2000, ApJ, 545, 1034

Spruit, H. C. 1996, in Evolutionary processes in binary stars, NATO ASI Series, 277 (Kluwer academic publishers), 249

Tafalla, M., Myers, P. C., Caselli, P., Walmsley, C. M., Comito, C. 2002, ApJ, 569, 815

van Dishoeck, E. F., \& Blake, G. A. 1998, ARA\&A, 86, 317

Walsh, A. J., Macdonald, G. H., Alvey, N. D. S., Burton, M. G., \& Lee, J.-K. 2003, A\&A, 410, 597

Wilson, T. L., \& Rood, R. T. 1994, ARA\&A, 32, 91

Wyrowski, F., Gibb, A. G., \& Mundy, L. 2002, ASP Conf. Proc., 267, ed. P. A. Crowther, 43

Zhang, Q., Hunter, T. R., \& Sridharan, T. K. 1998, ApJ, 505, L151 